Aleksandra Martyniak-Stronczek

USING SPIN POLARISED POSITIVE MUONS FOR STUDYING GUEST MOLECULE PARTITIONING IN SOFT MATTER STRUCTURES

INSTITUT FÜR PHYSIKALISCHE CHEMIE DER UNIVERSITÄT STUTTGART 


\title{
USING SPIN POLARISED POSITIVE MUONS FOR STUDYING GUEST MOLECULE PARTITIONING IN SOFT MATTER STRUCTURES
}

\author{
Von der Fakultät Chemie der Universität Stuttgart zur \\ Erlangung der Würde eines Doktors der Naturwissenschaften \\ (Dr. rer. nat.) genehmigte Abhandlung
}

vorgelegt von

\section{Aleksandra Martyniak-Stronczek \\ aus Gliwice, Polen}

Hauptberichter: Prof. Dr. Emil Roduner

Mitberichter: Prof. Dr. Frank Gießelmann

Tag der mündlichen Prüfung: 12.02.2007

INSTITUT FÜR PHYSIKALISCHE CHEMIE

DER UNIVERSITÄT STUTTGART

February 2007 



\section{TABLE OF CONTENTS}

TABLE OF CONTENTS....................................................................3

ACKNOWLEDGEMENTS..........................................................5

Chapter 1 INTRODUCTION.....................................................

1.1 General Introduction.............................................

1.2 Aim of the Work......................................................

Chapter 2 BACKGROUND AND FUNDAMENTAL ASPECTS...................11

2.1 Surfactants and Their Solutions......................................11

2.1.1 Definition and Classification of Surfactants......................11

2.1.2 Micellization..............................................17

2.1.3 The Hydrophobic Effect...................................19

2.1.4 Lyotropic Liquid Crystalline Mesophases.......................21

2.1.5 Interfacial Curvature...........................................24

2.2 The Role of Cosurfactants.........................................27

2.2.1 Effect on the Phase Behaviour.................................27

2.2.2 Fragrances and Flavours.....................................30

2.3 Muon Spin Resonance..........................................32

2.3.1 The Positive Muon, Muonium and Muoniated Radicals..............32

2.3.2 The ALC- $\mu$ SR Technique.....................................34

2.3.3 The Effect of Anisotropy....................................39

Chapter 3 EXPERIMENTAL DETAILS.........................................42

3.1 Sample Preparation.............................................. 42

3.1.1 DHTAC Dispersions Including Cosurfactants.....................42

3.1.2 Nickel Samples for Heisenberg Spin Exchange Experiments.........43

3.1.3 SDS Dispersions Including Cosurfactants.......................43

3.2 Determination of Phenyl Alcohol Solubility in Water....................45

3.3 Density Measurements............................................45

3.4 Differential Scanning Calorimetry ................................45

3.5 Polarized Optical Microscopy ...................................46 
3.6 Avoided Level Crossing Muon Spin Resonance.......................................46

3.6.1 The Experimental Setup....................................46

3.6.2 Data Analysis.............................................47

Chapter $4 \quad$ RESULTS AND DISCUSSION.....................................49

4.1 The Binary System of DHTAC....................................49

4.1.1 Solubility, Density and Calorimetric Measurements................50

4.1.2 Partitioning of Phenyl Alcohols................................52

4.1.2.1 Probe Molecule Location..............................52

4.1.2.2 Concentration Dependence............................58

4.1.2.3 Environment Polarity..................................59

4.1.3 Partitioning Equilibrium at the Water/Surfactant Interface..........64

4.1.3.1 The Classical Hydrophobic Effect.........................68

4.1.3.2 The Nonclassical Hydrophobic Effect.......................72

4.1.4 Information from Spin Exchange Experiments with $\mathrm{Ni}^{2+} \ldots \ldots \ldots . .73$

4.1.4.1 Determination of the Spin Exchange Rate Constant.........73

4.1.4.2 Dynamic Phenomena....................................75

4.1.5 Reorientational Dynamics of $\mathrm{MuC}_{6} \mathrm{H}_{5}\left(\mathrm{CH}_{2}\right)_{3} \mathrm{OH} \ldots \ldots \ldots \ldots \ldots \ldots . . . \ldots 77$

4.1.6 Conclusions..................................................81

4.2 Ternary and Quaternary Systems of SDS............................83

4.2.1 Identification of SDS Mesophases...............................84

4.2.2 Calorimetric Measurements......................................85

4.2.3 Partitioning of Phenyl Alcohols................................85

4.2.4 Effect of Dodecane Content....................................90

4.2.5 Reorientational Dynamics of $\mathrm{MuC}_{6} \mathrm{H}_{5}\left(\mathrm{CH}_{2}\right)_{3} \mathrm{OH} \ldots \ldots \ldots \ldots \ldots \ldots . . . . . . . .1$

4.2.6 Conclusions.....................................................92

Chapter 5 SUMMARY..........................................................94

Chapter 6 ZUSAMMENFASSUNG................................................99

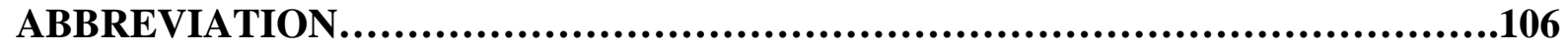

LIST OF FIGURES.................................................................108

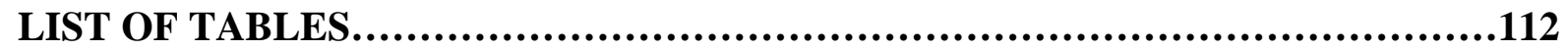

BIBLIOGRAPHY ....................................................................113 


\section{ACKNOWLEDGEMENTS}

I would like to express my deepest appreciation to my advisor Professor Emil Roduner for giving me the opportunity to work on this interesting project. I am very thankful for his guidance, permanent support and for sharing his knowledge, experience and time, all of which have contributed greatly in my mental development and confidence in facing challenges in research. I always had the freedom to follow my own ideas which I am very grateful for.

Taking the chair of the examination board by Prof. Dr. Dieter H. Wolf is kindly acknowledged. Writing the second advisory opinion by Prof. Dr. Frank Gießelmann is also kindly acknowledged.

I thank all my group colleagues who contributed to a pleasant atmosphere, in particular, Dr. Herbert Dilger for his assistance with ALC- $\mu$ SR measurements at PSI. Without him I would not have survived the beam times at PSI. I thank him very much for his infinite patience, help in calculations, and the valuable discussions on aspects of quantum mechanics. I also thank Dr. Iain McKenzie for his active support at PSI and interesting discussions on muonium chemistry.

I wish to thank Dr. Robert Scheuermann and Dr. Alexey Stoykov from the Laboratory for Muon Spin Spectroscopy at PSI for their technical support.

I thank Dr. Ian M. Tucker from Unilever Research and Development, United Kingdom, for teaching me how to prepare stable surfactant emulsions and for fruitful discussions on soft matter.

Special thanks go to Prof. Dr. A. Stephen K. Hashmi and to Dipl.-Chem. Tobias Burkert from the Institute of Organic Chemistry, Stuttgart University, for the synthesis of high purity para-propyl-2-phenylethan-1-ol. 
I would especially like to thank Dr. Jan Lagerwall from the Institute of Physical Chemistry, Stuttgart University for his help in the identification of SDS liquid crystal phases by POM and for valuable comments on thermotropic liquid crystals.

A very special thank you to Mrs. Gabriele Bräuning for ordering me reactants, her help in GC measurements and for preparation of a 3D illustration for the PCCP cover, to Mrs. Annette Hirtler for her assistance in sample preparation and for introducing to me aspects of instrumental analysis. I am grateful to both of them for their constant friendship.

I thank Mrs. Inge Blankenship who accounted for all the administration and contributed to a pleasant working atmosphere significantly.

The support by the mechanical, the electronical and the glassblower workshop is kindly acknowledged. I would like to mention especially Mr. Peter Haller and Mr. Walter Ottmüller from the mechanical workshop who built the $\mu$ SR sample cells for me.

Financial support by the European Commission under the 6th Framework Programme through the Key Action: Strengthening the European Research Area, Research Infrastructures. Contract ${ }^{\circ}$ : RII3-CT-2003-505925' is gratefully acknowledged.

Special thanks go to Dipl.-Chem. Barbara Vogel for her spiritual support, lively discussions, warm friendship and for Deutschunterricht.

And last but not least, my daughter Roksana and my dear friend Annette. For saving my soul I dedicate this work to both of them.

THANK YOU 


\section{Chapter 1}

\section{INTRODUCTION}

\subsection{General Introduction}

The unprecedented improvement in the quality of human life in Europe during the last 300 years have been the direct result of the individual freedom, technology, industry and economic growth that began to flower during the Industrial Revolution. For many millennia, humans relied on raw materials, especially hard matter. The eighteenth century was the age of cotton, wool, wood and iron. Dramatic development in the coal, steel and cement industry came in the $19^{\text {th }}$ century. One hundred years later inorganic materials and semiconductors were of main interest. The end of the $20^{\text {th }}$ century, however, was dominated by three massive scientific waves:

๑ nanoscale science;

口 information science;

a molecular biology.

This tendency caused the birth of a new class of engineered materials, soft matter substances [1,2]. In the past 10-20 years, research on colloids, polymers, foams, emulsions, microemulsions, liquid crystals and membranes, previously largely independent fields, have been integrated into the single field of soft matter.

In the first issue of the journal Soft Matter, the Nobel laureate Pierre-Gilles de Gennes characterizes soft matter as systems with "large response function” [3]. The most important feature of such soft material structures is that the ordering is intermediate between that of a crystalline solid and that of a liquid. They are distinguished from conventional liquids (solutions) due to their high viscosity and viscoelasticity. Another feature is the periodicity of the structures formed, typically in the range 1-1000 nm, which refers to nanoscale ordering. Moreover, a large number of internal degrees of freedom, weak interactions between structural elements, one, two and three-dimensional translational order, and delicate balance 
between entropic and enthalpic contributions to the free energy are common for various forms of these materials. In consequence, the described properties lead to large thermal fluctuations, macroscopic softness, metastable states and sensitivity of equilibrium structures to external conditions.

Such nanotechnologically enhanced materials have a wide range of applications and potential benefits in [4-12]:

a Biology and medicine;

- tissue engineering, drug delivery, diagnostics.

a Chemistry, environment and energy;

- catalysis, polymer synthesis, filtration, mineral flotation, foam blankets, solar cells, recycling of batteries.

๑ Agricultural products;

- $\quad$ pesticide, herbicide, fungicide.

口 Petroleum industry;

- fuel oil and jet fuel tank foams, fuel oil emulsions, asphalt emulsions.

a Manufacturing;

- $\quad$ paper making, ceramics, textiles, inks, paints, fire-fighting foams.

口 Information and communication;

- liquid crystal displays, novel optoelectronic devices, sensors.

口 Personal care and food products;

- cosmetics, detergents, ice cream, milk, creams, margarine.

Many properties of hard matter are now well understood, while we are on a learning curve with soft matter. For instance, chemical preparations are the basis of a whole range of modern soft materials in the home and at work. We see today that many everyday formulations involve surfactants and cosurfactants as irreplaceable ingredients in shampoos, shower gels, skin creams, polishes, washing powders, food and drinks, pharmaceuticals and bactericides, etc. Such chemical mixtures contain incredibly complex combinations of fragrance components mixed into the emulsions or microemulsions. Therefore, detailed understanding of what should go into formulations, the concentrations used and how each component functions is a basis to design modern “chemical cocktails” nowadays. Any chemical preparation has to fulfil many rigorous requirements, to be safe, healthy and biodegradable etc. Finally, it must remain as a coherent mixture for a reasonable period of time; it must be stable in physical, chemical and microbiological respect. A product with poor stability in 
which the components separate out, requiring an expiry date only a few weeks from the date of manufacture, which becomes cloudy or clumps of fungal growth adorn at its surface will not satisfy the customers. Hence, to optimise the properties of new commercial products it is essential to know the nature of soft matter substances.

It is predicted that exponential growth will occur in each of the nano-, info- and biosciences during the early part of the $21^{\text {st }}$ century, perhaps leading to a new Industrial Revolution.

\subsection{Aim of the Work}

The intention of this thesis is to explore the effect of guest molecules on the structure of diverse soft matter architectures, and thus the stability of the entire systems. More specifically, we focus on the determination of the factors that affect partitioning of fragrance components in emulsions and microemulsions, including various lyotropic liquid crystalline mesophases of cationic and anionic surfactants.

It is well known that the most common fragrance materials [13-15] are created based on the mixtures of aliphatic alcohols or/and phenyl alcohols. Until recently, however, qualitative and quantitative studies of the guest molecule partitioning into the surfactant membrane have been performed mostly for short, medium and long chain aliphatic alcohols. In consequence, in the literature arose a gap of information on the distribution of alcohols containing an aromatic group into colloidal surfactant dispersions.

Hence, to fill at least partly this gap, the probes used in this work are muoniated cyclohexadienyl radicals derived from five different phenyl alcohols. The variation of their hydrocarbon chain length changes their hydrophobicity, and this is expected to affect their partitioning between the aqueous and the hydrocarbon moiety of the surfactant dispersion, which is the main focus of the present study. Furthermore, a detailed understanding of solvation thermodynamic properties and reorientational dynamics of the chosen species, including Heisenberg spin exchange in order to confirm the interpretation of particular aspects, is also presented here.

Common methods for the investigation of a variety of soft matter structures, such as spherical micelles, vesicles, lamellar, rectangular, hexagonal and cubic phases or bicontinuous sponge type structures include light, X-ray and neutron scattering, and 
measurements of calorimetric and rheological properties. Nuclear magnetic resonance is a powerful tool for the study of the dynamics of specific molecules or groups, or also of the entire structures. For the same purpose but with higher sensitivity, fluorescent dye molecules or spin labels are introduced as guests or bound to the amphiphile in specific positions, permitting optical spectroscopy (UV-Vis) or electron spin resonance studies, respectively.

Technical applications may require other types of guest molecules to be introduced into these structures. Prominent examples are drug molecules for their delivery to specific sites in the body, or skin care, dyes and fragrance molecules in day care products or additives to food. They are often needed in low concentrations (because of the limited solubility or high toxicity, etc.) that makes it difficult to monitor them with conventional methods at a molecular level. For these reasons we used here a special experimental technique which permits highly sensitive spectroscopy, the Avoided Level Crossing Muon Spin Resonance (ALC- $\mu \mathrm{SR}$ ) [16-18]. ALC- $\mu$ SR relies on a unique property of the positive muon, i.e. where it becomes attached to a $\mathrm{C}=\mathrm{C}$ double bond or phenyl group via addition of the muonium atom $\mathrm{Mu}$, which can be regarded as a light hydrogen isotope. $\mathrm{Mu}$ addition to unsaturated molecules produces radicals that contain the muon as a fully polarized spin label which reveals information about the structure, kinetics, distribution and solvation thermodynamics of such radicals even under extremely dilute conditions in different colloidal dispersions. Moreover, the high spin polarisation and the single particle counting technique make the muon an exceptionally sensitive probe.

It has been already shown that ALC- $\mu S R$ has so far found applications for the investigation of muonium-substituted free radicals in various environments, for example in the gas phase [19], in polymers [20], in liquid crystals [21,22], in zeolites [23,24], and also for Mu in semiconductors [25] and for muons in metals [26].

This time, however, to prove that this method is also suitable to apply in more complex (multicomponent) systems than described above, the ALC- $\mu$ SR serves here as a tool for sensitive monitoring of the local environment and reorientational dynamics of muoniated radicals (kept at very low concentrations) in ternary and quaternary surfactant mixtures. Thus, it is a successive challenge of these studies to show that the muoniated radical can be treated as a spectator of the changes in the properties of diverse surfactant systems at a molecular level. 


\section{Chapter 2}

\section{BACKGROUND AND FUNDAMENTAL ASPECTS}

Basic principles of surfactants and their solutions, the role of cosurfactants, and the employed ALC- $\mu$ SR technique are the subjects of this Chapter. Examples of different types of amphiphiles are presented in the first Subsection. Micelle formation and the hydrophobic effect are considered in Subsection 2.1.2 and 2.1.3, respectively. However, the tendency for amphiphiles to aggregate into structures with distinct packings and interfacial curvatures is described in Subsection 2.1.4 and 2.1.5. The influence of alcohol on the phase behaviour including its role as a fragrance material is discussed in the second Section, whereas the theory of the ALC muon spin resonance is introduced at the end of this Chapter.

\subsection{Surfactants and Their Solutions}

\subsubsection{Definition and Classification of Surfactants}

The term surfactant is a blend of "surface active agent" and is used interchangeably with amphiphile, tenside (for synthetic surfactant), or, in the very old literature, with paraffin chain salts. Surfactants are usually organic compounds that are amphiphilic (from the Greek, amphis: both and philia: love, friendship) or amphipathic, i.e., they consist of one water preferring part, hydrophilic (their ionic or uncharged polar heads), and one oil preferring or water fearing part, referred to as hydrophobic or lipophilic (their hydrocarbon chains). Therefore, surfactants are typically sparingly soluble in both organic solvents and water, lowering the surface or interfacial tension of the medium in which they are dissolved. Soaps and detergents are also surfactants, or surfactant mixtures, whose solutions have cleaning properties. In some usage, surfactant is defined also as a "molecule capable of associating to form micelles" [12]. 
Surfactants are split into four groups according to the nature of their hydrophilic head: ionic, non-ionic, zwitterionic and amphoteric. There are, however, grey areas in such a system of classification. For instance, amphoteric surfactants can change to behave as cationics depending upon $\mathrm{pH}[7]$.

\section{Ionic Surfactants}

The head of an ionic surfactant carries a net charge. If the charge is negative, the surfactant is more specifically called anionic; if the charge is positive, it is called cationic. The polar or ionic head group usually reacts strongly with an aqueous environment via dipoledipole or ion-dipole interactions. The hydrophobic chain, however, reacts with a nonpolar environment via van der Waals interaction. It is interesting that the solubility of ionics increases rapidly in a narrow range, above a certain temperature, termed the Krafft point $\left(\mathrm{T}_{\mathrm{k}}\right)$. It is due to an interplay between the temperature-dependent solubility of amphiphiles and the temperature dependence of the CMC (Subsection 2.1.2) [4].

Soaps are anionic surfactants based on a long fatty chain, attached to a sulphate, carboxylate or phosphate group bearing a negative charge. This negative charge is countered by a positive ion, called the counter ion, which is a sodium ion but sometimes ammonium or potassium. Common types of anionic surfactants are shown in Table 1.

Table 1: Anionic surfactants.

\begin{tabular}{ll}
\hline Example & Structure \\
\hline Sodium dodecyl sulphate & $\mathrm{CH}_{3}\left(\mathrm{CH}_{2}\right)_{11} \mathrm{OSO}_{3}{ }^{-\mathrm{Na}^{+}}$ \\
Sodium dodecylbenzene & $\mathrm{CH}_{3} \mathrm{CH}_{2} \mathrm{CH}_{2} \mathrm{CH}_{2} \mathrm{CH}_{2} \mathrm{CH}_{2} \mathrm{CH}_{2} \mathrm{CH}_{2} \mathrm{CH}_{2} \mathrm{CHCH}_{2} \mathrm{CH}_{2} \mathrm{CH}_{2} \mathrm{CH}_{3}$ \\
sulfonate & \\
Sodium lauryl ether sulphate & $\mathrm{CH}_{3}\left(\mathrm{CH}_{2}\right)_{10} \mathrm{CH}_{2} \mathrm{OCH}_{2} \mathrm{CH}_{2} \mathrm{OCH}_{2} \mathrm{CH}_{2} \mathrm{OSO}_{3}{ }^{-\mathrm{Na}^{+}}$ \\
\hline
\end{tabular}


A good example is sodium dodecyl sulphate (SDS or NaDS), also known as sodium lauryl sulphate (SLS) which is used in household products such as shampoos, toothpaste, shaving foams and bubble bath for its thickening effect and its ability to create a lather. An important advantage of this kind of amphiphile is that does not react with lime, producing insoluble salts with calcium and so does not suffer loss of activity in hard water.

However, sodium dodecylbenzene sulfonate (SDBS) is used as hand dish-wash liquids. Nowadays many anionics contain ethylene oxide groups to provide the hydrophilic part that enhance the attraction to water. Therefore, sodium lauryl ether sulphate with three ethylene oxide groups has excellent degreasing properties and is a good foam producer, being a common component of modern shampoos and bubble bath products, etc. In general, the smaller amphiphilic molecules make good hydrotropes (short chain lipophiles) while the larger molecules are better at detergency, where their anionic head enables the molecule to be electrostatically attracted to solid particles of soil, and thus facilitating their removal [7].

Anionics also have the disadvantage that they work only in alkaline conditions, where $\mathrm{pH}$ should be greater than 8 . At low $\mathrm{pH}$ values, the soap breaks down forming an insoluble fatty acid. Moreover, anionic surfactants cannot be used in conjunction with cationic surfactants which would result in them being attracted and chemically combining with subsequent loss of activity of both [7].

In our work we used one of the most well studied surfactant, sodium dodecyl sulphate (SDS), which is described above.

Cationic surfactants are another type of ionic surfactant. In this case the headgroup is often based around a quaternary ammonium ion in which four alkyls are attracted to a positive charged nitrogen atom (hence the name quaternary). In comparison to anionics, cationics are made in much smaller quantities due to their high aquatic toxicity, poor detergency capacity and relatively high price. However, many materials, such as hairs, skin, fibres and car paintwork carry negative charges when wet, and thus attract cationic surfactants [7]. More specifically, a cationic surfactant becomes chemisorbed to negative groups in the protein (keratin) that form the hair shaft. The protein is coated in cationic surfactant in which the hydrophobic chains orient away from the negatively charged surface. In effect, the surface nature is changed from that of a protein one to a hydrocarbon one. Washed and dried hair is softer, silky, glossy, and also does not suffer the static electricity phenomena that causes flyaway hair when combed. A common cationic used in shampoos and in hair conditioners is lauryl trimethyl ammonium chloride (see Table 2). 
Table 2: Cationic surfactants.

\begin{tabular}{|c|c|}
\hline Example & Structure \\
\hline $\begin{array}{l}\text { Lauryl trimethyl ammonium } \\
\text { chloride }\end{array}$ & $\mathbf{C H}_{3}\left(\mathbf{C H}_{2}\right)_{10} \mathbf{C H}_{2}-\left.\right|_{\mathbf{C H}_{3}} ^{\mathrm{CH}_{3} \mathbf{C l}^{\Theta}} \mathbf{C H}_{3}$ \\
\hline Cetyl pyridinium choride & $\begin{array}{r}\mathrm{CH}_{3}\left(\mathrm{CH}_{2}\right)_{14} \mathbf{C H}_{2} \mathbf{N} \\
\Theta\end{array}$ \\
\hline $\begin{array}{l}\text { Cetyl trimethyl ammonium } \\
\text { chloride }\end{array}$ & $\mathbf{C H}_{3}\left(\mathbf{C H}_{2}\right)_{14} \mathbf{C H}-\left.\right|_{\mathbf{C H}_{3}} ^{\mathrm{CH}_{3}} \mathrm{Cl}^{\Theta} \mathbf{C H}_{3}$ \\
\hline
\end{tabular}

Similarly, when cationic molecules adsorb into skin, a protein acquires a pleasant softness.

Fabric conditioners work in the same way, make the fibres of the fabric softer and simultaneously easier to iron.

The glossy effect, however, of vesicle paintwork can be achieved by using car shampoos containing cationic surfactants.

Finally, cationics can bond to the proteins of the cell membrane and prevent it functioning, causing the bacteria to perish. Unfortunately, such a destructive action can intrude into other biological systems, for instance to eyes. In consequence, the adsorption of cationics onto lenses made of protein causes fogging. Cetyl trimethyl ammonium chloride is a well known algaecide and bactericide, while cetyl pyridinium chloride is used as a mouthwash.

In this thesis 2,3-diheptadecyl ester ethoxypropyl-1,1,1-trimethylammonium chloride (abbreviated as DHTAC) was used, being an cationic dichain surfactant (see Figure 1). 


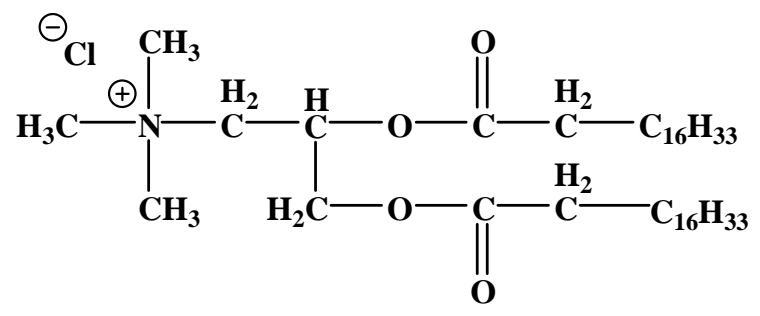

Figure 1: Structure of DHTAC.

\section{Non-ionic Surfactants}

Non-ionic surfactants are not sensitive to hard water, they have excellent emulsifying properties and are usually compatible with other types of surfactant. Therefore, non-ionics production is approaching that of anionic surfactants, with a growth curve that is increasing faster. Most of non-ionic molecules have as their hydrophilic head a series of ethylene oxide groups in the form of an ethoxylate tail. The surfactant with only two ethylene oxide groups will not be dissolved in water, but instead will be oil soluble, whereas the surfactant with twenty such groups will have good water solubility. An interesting property of non-ionic ethoxylates dissolving by means of hydrogen bonding is that they come out of solution (known as the cloud point) on heating as the thermal energy becomes to break these hydrogen bonds and reduce solubility [7]. This phenomenon is valid, however, only at elevated temperatures, hence just below their cloud points, they are particularly effective. For instance, nonyl phenol ethoxylate (see Table 3) used as household and idustrial cleaners, reaches the cloud point at $82^{\circ} \mathrm{C}$.

Table 3: Non-ionic surfactants. (EO) denotes ethylene oxide and (PO) propylene oxide to give EO/PO copolymers. The number of mols of ethylene oxide per mole of lipophile is indicated by the number followed by EO.

\begin{tabular}{|c|c|}
\hline Example & Structure \\
\hline $\begin{array}{l}\text { Nonyl phenol ethoxylate } \\
\text { (9EO) }\end{array}$ & $\mathrm{CH}_{3}\left(\mathrm{CH}_{2}\right)_{8} \mathrm{C}_{6} \mathrm{H}_{4} \mathrm{O}\left(\mathrm{CH}_{2} \mathrm{CH}_{2} \mathrm{O}\right)_{8} \mathrm{CH}_{2} \mathrm{CH}_{2} \mathrm{OH}$ \\
\hline $\mathrm{EO} / \mathrm{PO}$ block polymers & $\mathrm{HO}\left(\mathrm{CH}_{2} \mathrm{CH}_{2} \mathrm{O}\right)_{\mathrm{a}}\left(\mathrm{CH}_{2} \mathrm{CH}_{2} \mathrm{CH}_{2} \mathrm{O}\right)_{\mathrm{b}}\left(\mathrm{CH}_{2} \mathrm{CH}_{2} \mathrm{O}\right)_{\mathrm{c}}-$ \\
\hline
\end{tabular}


In production of low foam machine wash liquids, synthetic EO/PO block polymers are needed, where the lipophile is represented by PO units and the hydrophile by repeated EO units as shown in Table 3.

\section{Zwitterionic Surfactants}

Surfactants containing both positive and negative charges in the head group are called zwitterionics. Usually the positive charge is associated with an ammonium group and the negative charge is often a carboxylate. They are used in cosmetics, since they have been found to be non-irritants for skin and eyes.

\section{Amphoteric Surfacatnts}

Amphoterics have a similar nature as a previous group of surfactants. They can be, however, distinguished from zwitterionics because they pass from the cationic to the anionic form on increasing $\mathrm{pH}$, while in the case of zwitterionic surfactants only the stability changes. An amphoteric such as coco dimethyl betaine (Table 4) offers good detergency coupled with high foaming capacity and mild action on the skin. Hence, it is valuable in products such as baby shampoos and shower gels, etc.

Table 4: Amphoteric surfactant.

\begin{tabular}{ll}
\hline Example & Structure \\
\hline Coco dimethyl betaine & \\
$\mathbf{R}$ - coco fatty group
\end{tabular}

\section{Surfactants in the Future}

Structure modifications of surfactants, such as varying the molecules chain length or putting in ethylene groups can significantly influence their properties. It seems, however, that new generations of amphiphiles require more radical changes in their structure. A particular example of a new breed of surfactants is the Gemini surfactant (GS) [27], a so-called dimeric surfactant being a single molecule with at least two polar or ionic hydrophiles and two 
lipophiles (twin-like structure). The two hydrocarbon tails can be short or long, while the two polar heads can be anionic, cationic or nonionic. The spacer can be attached directly to the identical ionic groups (Figure 2a), alternatively, the two identical amphiphiles are joined midway (Figure 2b). The spacer length can be short (2 methylene groups) or long (12 methylene groups); rigid (stilbene) or flexible (methylene chains) [28]. The GS with three or more polar groups or chains (trimer, tertramer) are also known.
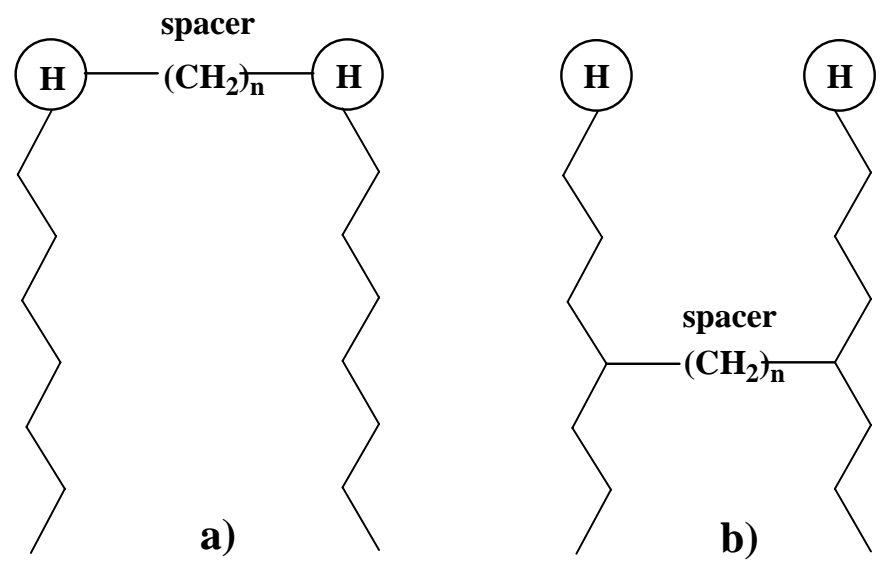

Figure 2: Two types of Gemini surfactants. H denotes ionic or nonionic headgroup. Adopted from [7].

Geminis can self assemble at much lower concentrations and are superior in surface activity as compared to conventional surfactants [27,28]. GS with short hydrophobic spacers (increased intermolecular repulsion between heads) form thread like micelles and those with long spacer form rod like micelles. Moreover, dimeric and trimeric surfactants can also form stable vesicles.

One area in which Geminis show promise is as vesicle for delivering bioactive molecules into living cells [7]. GS are also attractive for skin care formulations, catalysis, solubilization processes, paint additives and biotechnology.

\subsubsection{Micellization}

In aqueous solution dilute concentrations of surfactant act much as normal electrolytes, but at higher concentrations, surfactant shows very different behaviour. 
As is presented in Figure 3 amphiphiles can either adsorb at the air/water or the oil/water interface, forming a monolayer, or they can aggregate into structures in which the hydrophobic portions are oriented within the cluster and the hydrophilic portions are exposed to the solvent. Such aggregates are called micelles, which are the simplest of amphiphilic selforganizing structures.

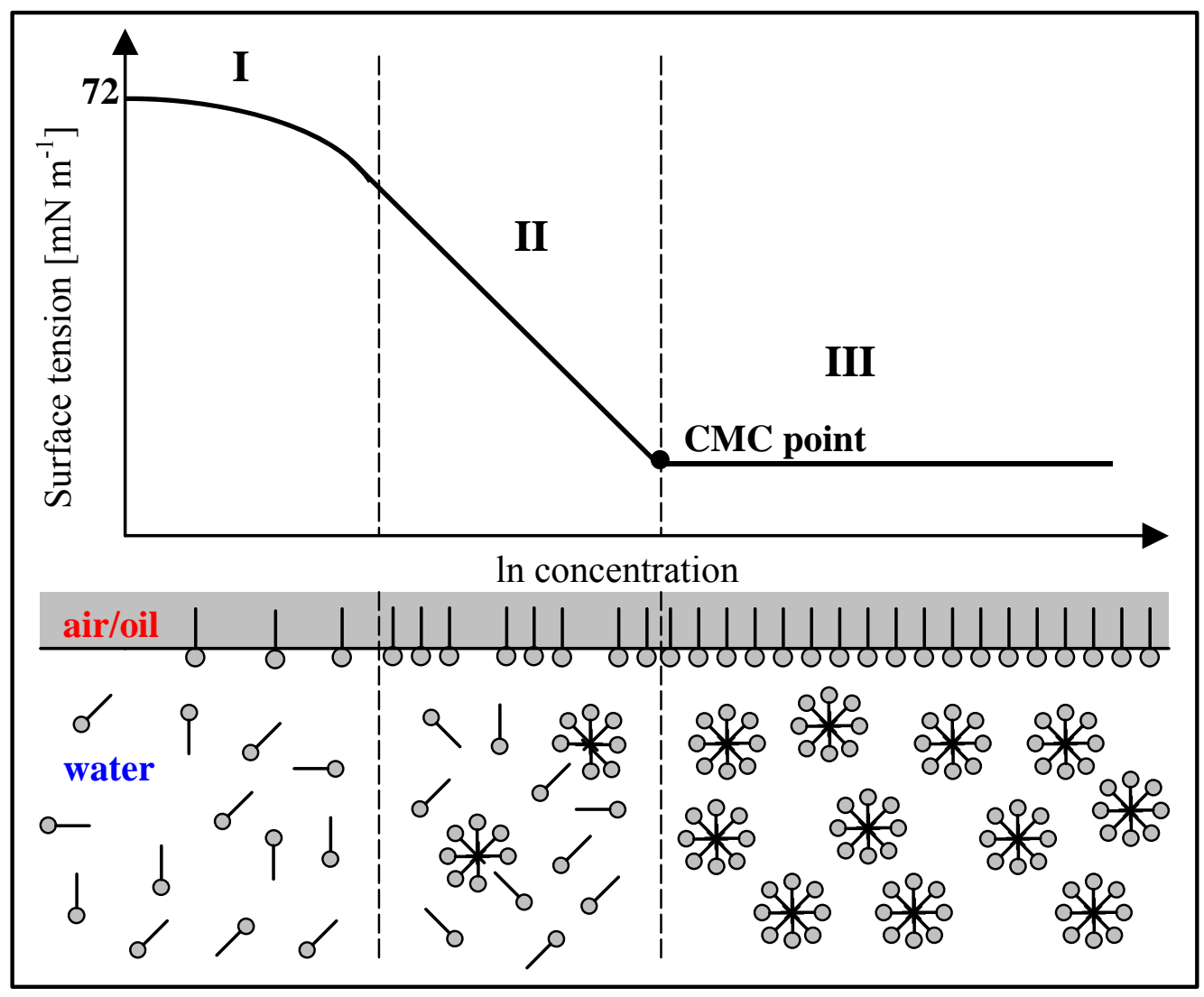

Figure 3: Variation of surface tension with concentration (on a logarithmic scale) for a pure aqueous surfactant solution.

In regime I, at low surfactant concentrations a gradual decay in surface tension is observed which corresponds to an increase in the surface excess (defined as the concentration of surfactant molecules in a surface plane, relative to that at a similar plane in the bulk) of surfactant molecules.

In regime II, the surface becomes crowded with surfactant. The other amphiphiles arrange into micelles, and thus the surface tension decreases rapidly from $\gamma=72 \mathrm{mN} \mathrm{m}^{-1}$ (for pure water) until a point where $\gamma$ is almost independent of concentration. This point is (for an isothermic process) is called the critical micelle concentration (CMC) [29-32]. Such unassociated surfactant molecules coexisting with micelles are often called unimers [4]. The 
micelles in this region are unstable and relax, releasing surfactant molecules in order to redress the equilibrium [33]. Thus the micelles behave like reservoirs of monomers that try to maintain the volume fraction of surfactant molecules at the CMC [33].

In regime III, at high surfactant concentration (above the CMC) the surface tension is nearly constant. This is attributed to the saturation of the surface excess due to formation of a complete monolayer at the CMC. The limiting value of $\gamma$ above the CMC is typically $35 \mathrm{mN}$ $\mathrm{m}^{-1}$.

The CMC is dependent on surfactant type, chain length, temperature, and addition of salt and cosolutes.

\subsubsection{The Hydrophobic Effect}

Hydrophobicity generally relates to the thermodynamics of mixing nonpolar substances with water and to the free energy involved in transferring apolar solutes from a nonpolar environment into water. It plays a key role in many biological processes, such as micelle formation, protein folding, and also in partitioning of drugs, metabolites, and toxins into these membranes. In technical applications it often dominates processes such as coagulation and detergency, wetting and aggregation at interfaces [34,35]. Examination of the literature reveals that the molecular origin of solvation of nonpolar species is based on two concepts: the classical and nonclassical hydrophobic effect.

\section{The Classical Hydrophobic Effect}

The molecular interpretation of the classical version of the hydrophobic effect consists of two contributions: hydrophobic hydration and hydrophobic interaction [36-38] focuses on the effect on water structure in the presence of nonpolar moieties [39].

\section{Hydrophobic Hydration}

The way a nonpolar solute affects the local structure of water in its immediate environment is called hydrophobic hydration $[35,40]$.

For instance, a thermodynamic description of the micelle formation in aqueous solutions involves the classical concept [4,30] Micellization is predominantly an entropic effect, as deduced from comparison of the entropy and enthalpy contributions to the Gibbs free energy 
of the micelle formation. An increase in entropy of the system of amphiphiles in micelles results from losing tetrahedral arrangement of hydrogen-bonded water molecules. The gain in entropy of water outweighs the enthalpy penalty (favourable interaction between the surfactant hydrocarbon chains) and the loss of configurational entropy of the amphiphiles upon the constraints imposed by the micellar structure.

Many previous studies $[36,42,43]$ have shown that hydrophobic hydration of noble gases, alkanes and aliphatic alcohols, and the transfer of such nonpolar compounds between water and bulk oil environments, are also governed by the 'classical' hydrophobic effect. In contrast to micelle formation, these phenomena are characterised by the large negative entropy that scales with the number of $\mathrm{CH}_{2}$ groups. It is attributed to the excluded volume effect (exaggerated by the small size of water molecules) associated with the reversible work necessary to create a cavity to accommodate a solute in water (the effects associated with the difference in volume of the two phases are excluded), and not with the iceberg model proposed by Frank and Evans [44,45]. Furthermore, the entropic contribution often compensates to a large extent the enthalpic contribution to the Gibbs free energy of solvation (see Subsection 4.1.3). Finally, large and positive hydration heat capacity change attributed to the reorganization of H-bonds of water molecules in the hydration shell large is a successive evidence for the hydrophobic hydration.

\section{Hydrophobic Interaction}

Kauzmann introduced the concept of hydrophobic interaction in 1959. The term hydrophobic interaction denotes the tendency of relatively apolar molecules to stick together in aqueous solution $[35,38]$.

Hydrophobic interaction has been broken down into pairwise, bulk and enforced interactions. Hydration of nonpolar molecules in the water phase where the solute concentration is below the CMC is a typical pairwise interaction. Hydrophobic bulk interaction, however, occurs above the CMC of the solute. Finally, when a chemical reaction happens in the considered system the enforced interaction appears.

The negative values of enthalpy associated with the direct solute-water van der Waals or/and H-bond interaction energy are hallmarks of the hydrophobic interaction.

\section{The Nonclassical Hydrophobic Effect}

On the other hand, Wimley and White [46] found that a 'nonclassical' hydrophobic effect is operative in the partitioning of nonpolar solutes into lipid double layers. It is associated 
with a further decrease of the negative enthalpy of transfer upon the penetration of the solute into the membrane, indicating that it participates in the lipid-lipid interaction and enhances it, which is called the 'bilayer effect' $[39,47]$. A negative increment of the enthalpy and entropy of transfer per additional methylene group is characteristic of partitioning into the membrane $[39,46,47]$.

Another feature of such a hydrophobic process is the large negative heat capacity change upon partitioning, indicating a major contribution from the dehydration of nonpolar solute moieties.

In addition, chemical nature, a size and a polarizability of the solute play a decisive role in determining the exact value of solvation Gibbs energy change in the case of both the classical and the nonclassical concept of the hydrophobic effect.

\subsubsection{Lyotropic Liquid Crystalline Mesophases}

Liquid crystals were detected for the first time in 1888 by Friedrich Reinitzerin. They are considered as a fourth state of matter sharing features of crystalline solid and normal liquids: the degree of molecular ordering is between that of a liquid and a crystal and in terms of rheology the systems are neither simple viscous liquids nor crystalline elastic solids. Certain of these phases have at least one direction that is highly ordered so that liquid crystals exhibit optical birefringence $[8,48]$. Through this intermediate nature, liquid crystals are also termed mesophases (from the Greek meso: intermediate).

There are two main varieties of liquid crystals, thermotropic and lyotropic. Thermotropic mesophases are composed of only one stiff elongated component, and their stability is a function of temperature. In contrast, the materials that form the liquid crystals by the addition of solvents are called lyotropic liquid crystals, which normally consist of two or multicomponent systems. Moreover, their liquid crystallinity is controlled by concentration.

The most common lyotropic liquid crystals are those formed by surfactant where the solvent is usually water $[49,50]$. The main structures associated with two-component surfactant-water systems are shown in Figure 4.

At high concentrations, micelles fill space by packing in a cubic array ( $\mathrm{I}_{1}$ phase) as presented in Figure 4a. 


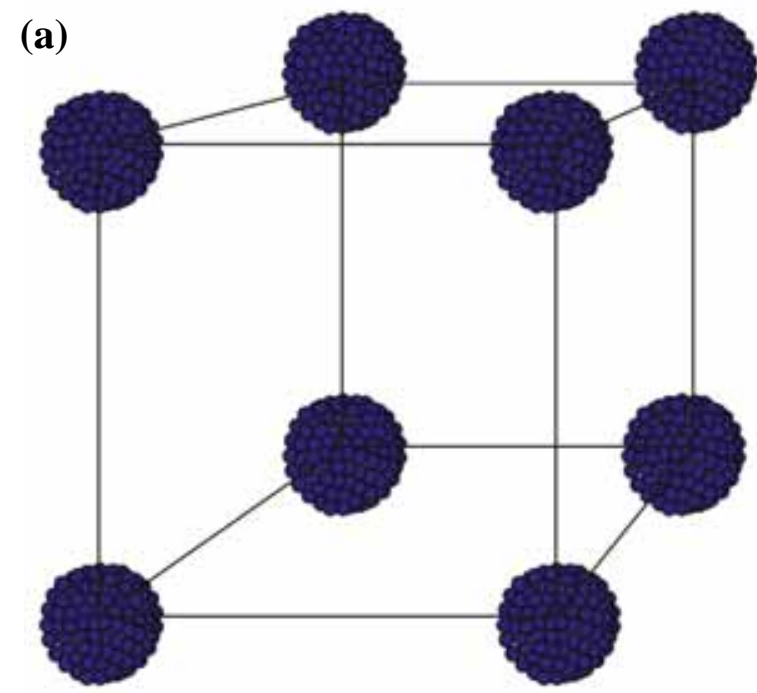

(b)

(c)
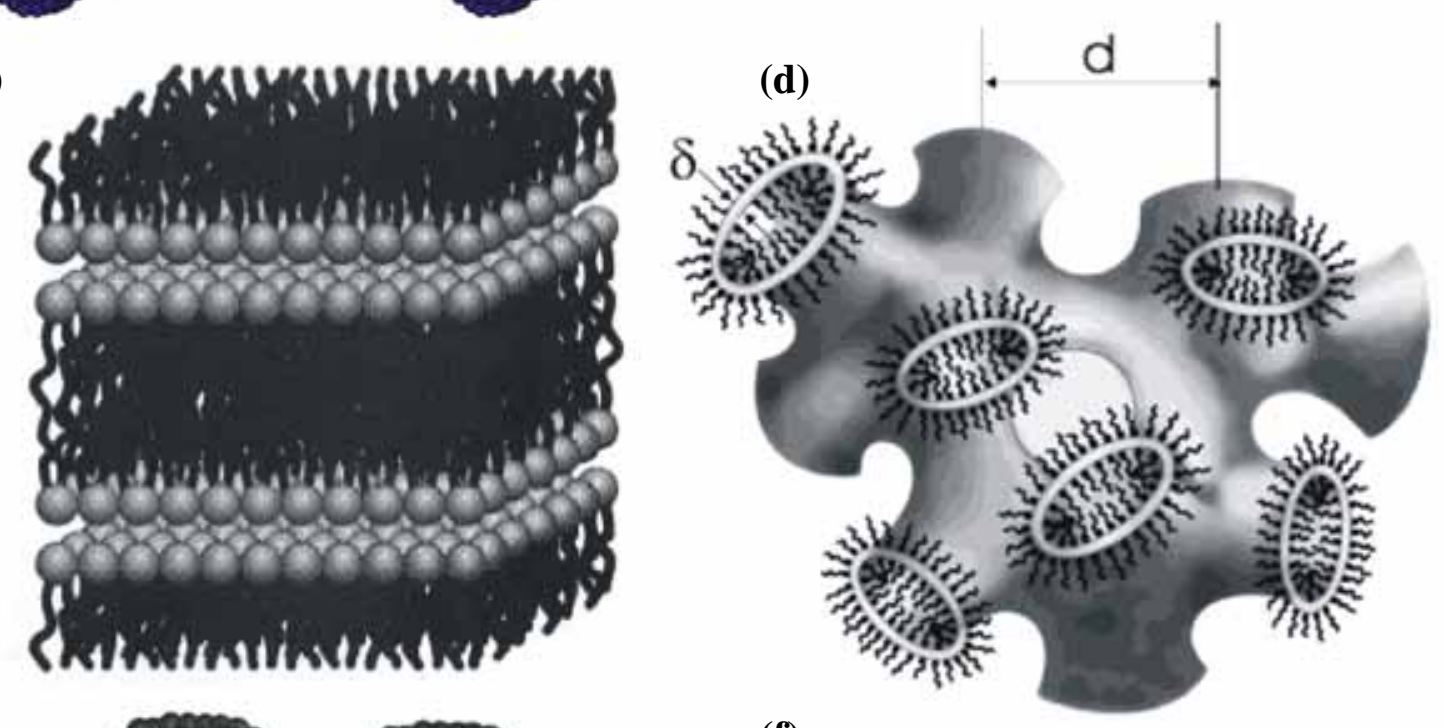

(e)

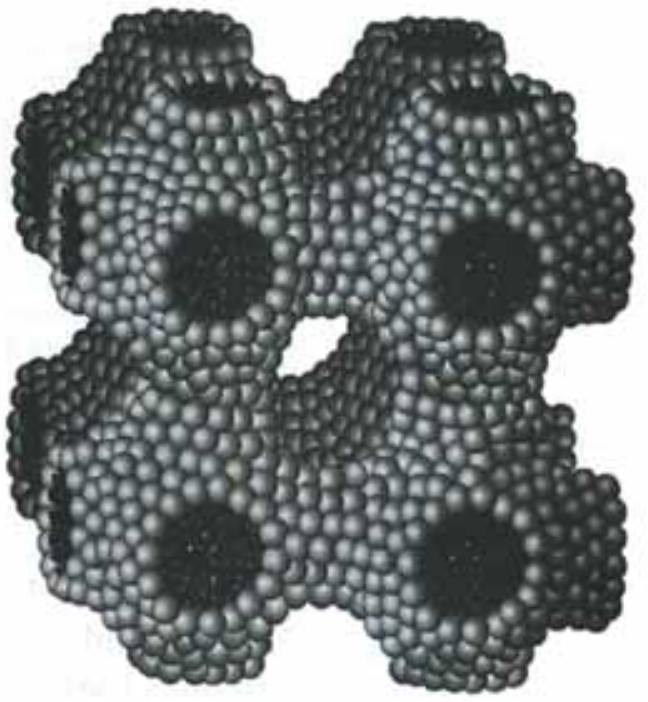

(f)

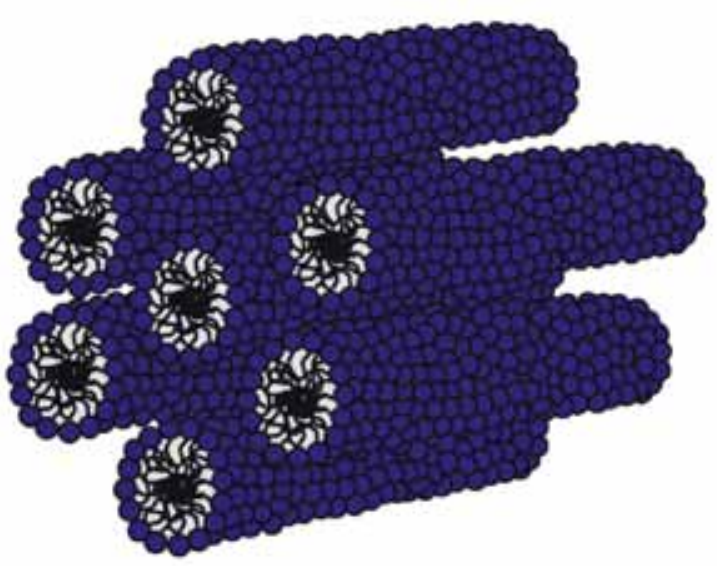

)

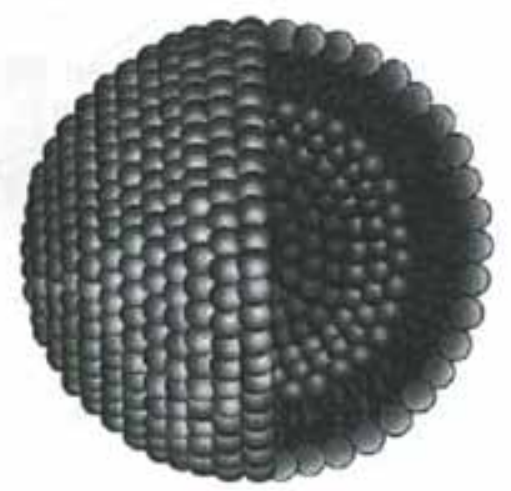

Figure 4: Common lyotropic liquid crystalline mesophases: (a) normal micellar cubic phase $\left(\mathrm{I}_{1}\right)$, (b) normal hexagonal phase $(\mathrm{E})$, (c) normal lamellar phase $\left(\mathrm{L}_{\alpha}\right)$, (d) inverted sponge phase $\left(\mathrm{L}_{3}\right)$, (d) normal bicontinuous cubic phase $\left(\mathrm{V}_{1}\right)$, (f) shell like structure of unilamellar vesicles, where $\delta$ denotes the thickness of the water layer and $d$ refers to the cell size of the membrane. Figure 4c, e, f adopted from ref. [51], and Figure 4d from ref. [52]. 
Further increase in surfactant number leads to the formation of normal columnar hexagonal $\mathrm{E}$ structure composed of long cylindrical micelles in circular cross-section arranged on a 2D hexagonal lattice (see 4b).

However, the parallel elliptical cylinder packings gives rise to the two-dimensional columnar rectangular phase being intermediate in the transition from hexagonal columnar to lamellar organization.

As is shown in Figure 4c, bilayers separated by water tend to stack into a normal lamellar phase. A phospholipid bilayer knows many different phases [53]. At low temperature, the subgel $\mathrm{L}_{\mathrm{c}}$ with highly ordered and tilted hydrophobic chains appears (Fig. 5a). Upon heating the subgel transforms to a lamellar $\mathrm{L}_{\beta}$ or $\mathrm{L}_{\beta^{\prime}}$ gel phase (Fig. 5b, c), which are more hydrated than the $\mathrm{L}_{\mathrm{c}}$ phase. Even though, the chains are in the solid-like all-trans conformation.

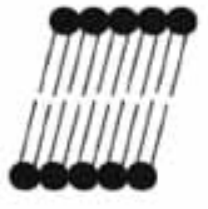

(a) $\mathrm{L}_{\mathrm{c}}$

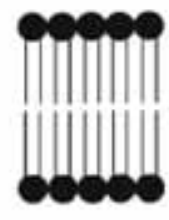

(b) $\mathrm{L}_{\beta}$

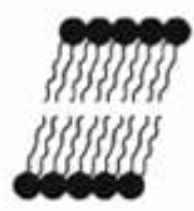

(c) $\mathrm{L}_{\beta^{\prime}}$

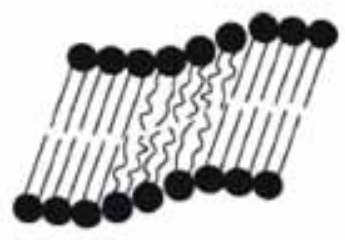

(d) $\mathrm{P}_{\beta^{\prime}}$

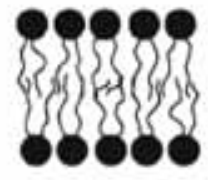

(e) $\mathrm{L}_{\alpha}$

Figure 5: Various bilayer phases. Adopted from [53].

At higher temperature, the gel undergoes a transition to the $\mathrm{L}_{\alpha}$ phase (Fig. 5e), the so-called liquid crystalline or fluid phase, where $\alpha$ stands for liquid-like, disordered chains with different conformations. The ripple phase [54] however, is a coexistence between the $\mathrm{L}_{\mathrm{c}}$ or $\mathrm{L}_{\beta^{\prime}}$ phase and $\mathrm{L}_{\alpha}$ phase (Fig. 5d).

In the case, when the polar solvent becomes the dominant component, inverse structures are favourable. The reverse packing of amphiphiles is illustrated in Fig. $4 \mathrm{~d}$ for the $\mathrm{L}_{3}$ sponge phase, in which a random network of very flexible membranes is isolated by water. Commonly, the sponge phase is considered as a disordered and less viscous version of the cubic phase, the so-called "molten lyotropic mesophase" [48]. Another example of molten liquid crystals is a microemulsion defined as a system of water, oil and an amphiphile which is a single optically isotropic and thermodynamically stable liquid solution. In some respects, microemulsions can be considered as small-scale versions of emulsions, i.e., droplet type dispersions either of oil-in-water $(\mathrm{o} / \mathrm{w})$ or of water-in-oil (w/o), with a size range in the order of 5-50 $\mathrm{nm}$ in drop radius [55]. 
Mesophases based on saddle-splay surfaces are normal bicontinuous cubic $\mathrm{V}_{1}$ structures (Fig. $4 \mathrm{e}$ ), which are composed of two continuous channels of water, separated by a surfactant bilayer. Bicontinuous cubic phases with four and sixfold connectors are also known [48]. A vesicle, however, is formed by small regions of bilayers closing back on themselves to form a hollow spherical structure where the interaqueous compartment is separated from the surroundings (Fig. 4f). A vesicle consisting of single bilayer is termed a unilamellar vesicle, whereas with a shell of several bilayers is called a multilayer vesicle.

Lyotropic mesophases can be identified to a certain extent based on textures observed using polarized light microscopy. The lamellar, hexagonal and rectangular mesophases are birefringent having different characteristic textures, while microemulsions and all cubic phases are optically isotropic and cannot be distinguish from one another. Sponge mesophases, however, are characterized by flow birefringence (giving anisotropic textures), yet they are isotropic at rest. Identification of liquid crystals can be most readily done by using small angle $\mathrm{x}$-ray scattering (SAXS), small angle neutron scattering (SANS) or ${ }^{2} \mathrm{H}$ NMR on solutions in $\mathrm{D}_{2} \mathrm{O}$.

\subsubsection{Interfacial Curvature and Packing Parameter}

A precise description of the topology of any mesostructures comes from the curvatures of the interface lying at the boundaries of immiscible domains. The position of each point on the membrane surface can be described by the two fundamental types of curvatures: mean and Gaussian curvatures [4,56] expressed in terms of the principal curvatures: $c_{1}=1 / R_{1}$ and $c_{2}=$ $1 / R_{2}$, where $\mathrm{R}_{1}$ and $\mathrm{R}_{2}$ are the radii of curvature in two perpendicular directions, as shown in Figure $6[4,51]$.

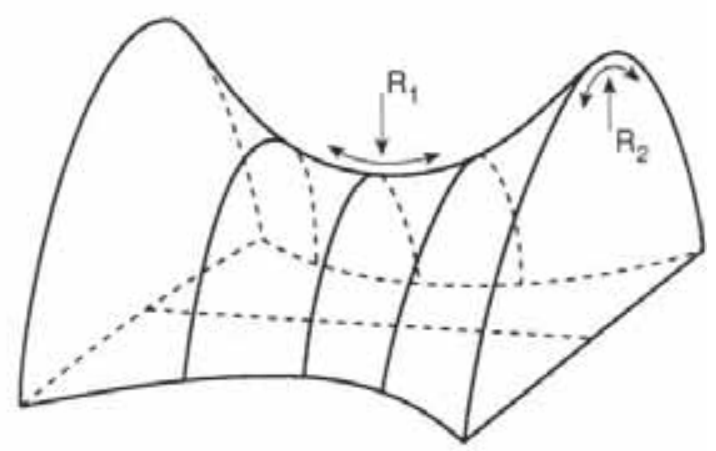

Figure 6: Principal radii of curvature of a saddle surface in three dimensions. Adopted from [51]. 
Hence, the mean curvature is:

$$
H=\frac{1}{2}\left(\frac{1}{R_{1}}+\frac{1}{R_{2}}\right)
$$

whereas, the Gaussian curvature is given by:

$$
K=\frac{1}{R_{1}} \times \frac{1}{R_{2}}
$$

To assign a sign of the radii of these curvatures (which defines the type of the mesophase), a normal direction to the surface must be specified. By convention, the radius vector is positive pointing toward the polar, water region and vice versa.

The product of these interfacial curvatures determines intrinsic geometry:

- both convex or concave curvatures lead to an elliptic cap (spherical micelles);

- one vanishing curvature results in parabolic shape (cylindrical micelles);

- both vanishing curvatures give a planar structure (lamellae);

- both opposite curvatures lead to hyperbolic surface (cubic bicontinuous).

Furthermore, the sum of contributions of mean and Gaussian curvature gives the elastic free energy density which is associated with surface curvature for small deformations:

$$
F_{\mathrm{el}}=F_{\text {mean }}+F_{\text {Gauss }}=\frac{1}{2} \kappa\left(c_{1}+c_{2}-c_{0}\right)^{2}+\bar{\kappa}\left(c_{1} c_{2}\right)
$$

where $c_{0}$ is the spontaneous curvature, and the quantities $\kappa$ and $\bar{\kappa}$ are the elastic moduli for mean and Gaussian curvatures, respectively. They can be measured by light scattering, providing in this manner information about the flexibility of surfactant films. Uncharged surfactant films, for example, have elastic energies $F_{\text {el }} \leq k_{\mathrm{B}} T$, meaning that they are quite flexible.

A comparable, although quantitatively different approach to analyse the nature of amphiphile self-assembly uses a surfactant packing parameter $P$ concept. As is shown in Figure 7 , the surfactant packing parameter is controlled by the effective area of the head group $a$, the hydrophobic chain of length $l_{\mathrm{c}}$, and molecular volume $v_{\mathrm{c}}$.

Thus, the surfactant packing parameter is expressed as follows:

$$
P=\frac{v_{\mathrm{c}}}{a l_{\mathrm{c}}}
$$




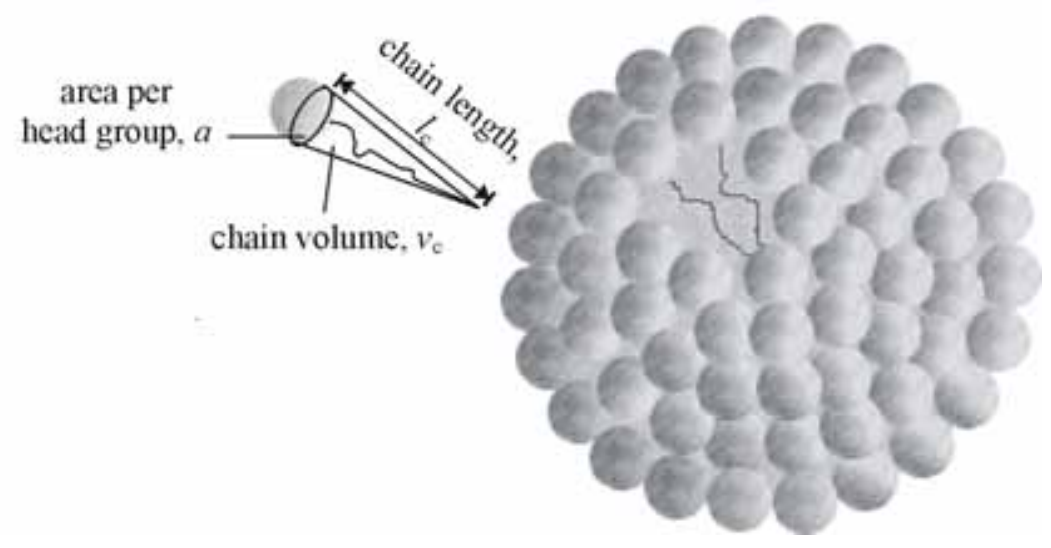

Figure 7: The packing of surfactant molecules in a spherical micelle.

The correlation between amphiphile shape and mesophase type is illustrated in Figure 8 . In A, inverted cone-type molecules, with packing parameters $P>1$ form inverted micellar structures, where the hydrophilic head groups are confined in the interior of the aggregate. As the cylinder-shaped molecules have $P=1$, a planar interface is preferred (see B). Cylindrical micelles and hexagonal phases are formed when $1 / 3<P<1 / 2$. However, surfactants with $P<$ 1/3 imply normal spherical micelles as presented in C. In the case, when the monomers have complementary shapes, surfactant mixtures can also organize into bilayers (see D).

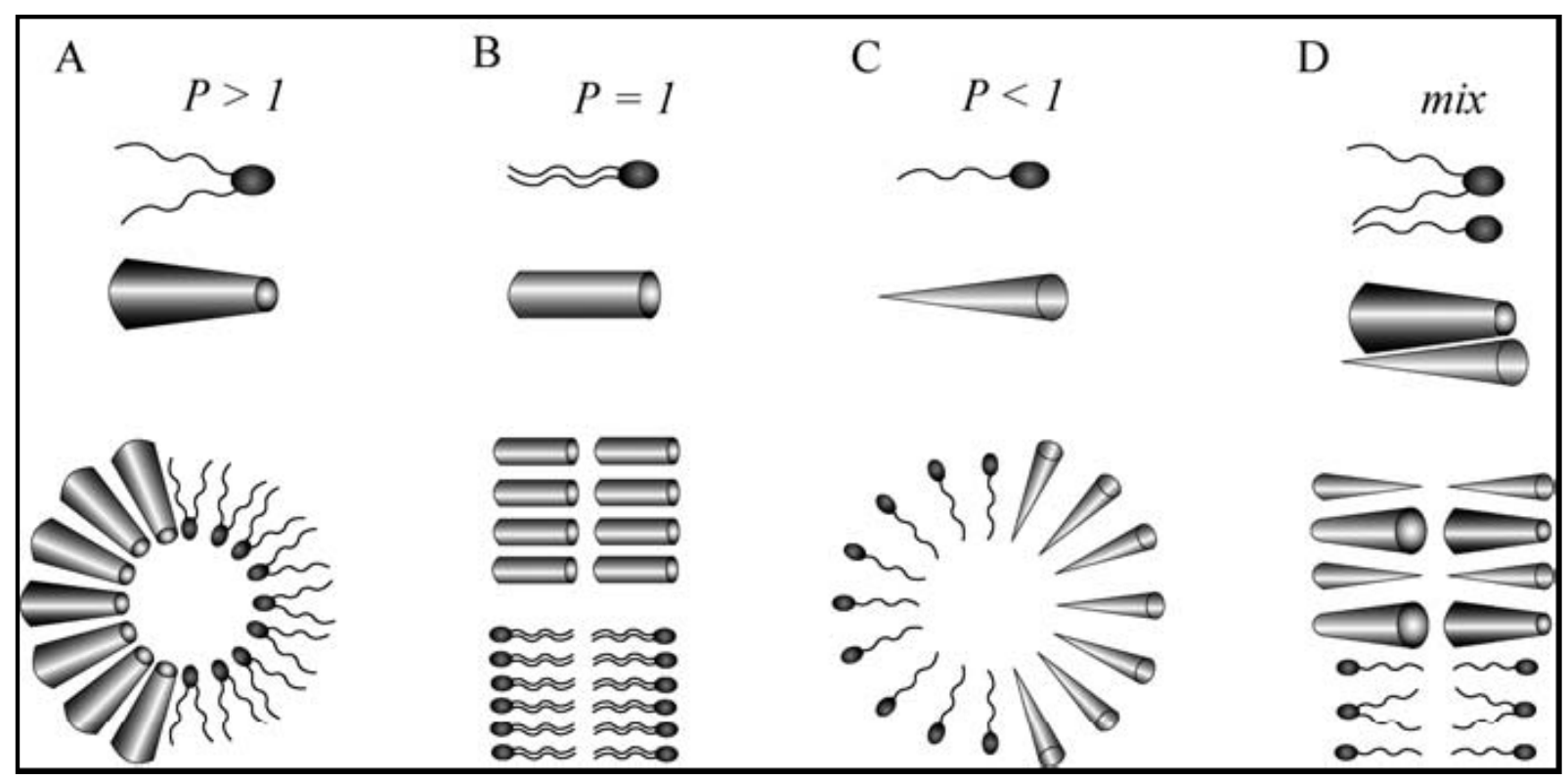

Figure 8: Surfactant packing parameter for various aggregate structures. Adopted from ref. [57]. 
It can be noted that a decrease in the surfactant packing parameter corresponds to an increase in mean curvature, revealing a strong relationship between these factors.

\subsection{The Role of Cosurfactants}

Many applications, ranging from laundry detergents to microemulsions for oil recovery, make use of combinations of surfactant and cosurfactant. Among the very large number of cosurfactants (so called surface active additives or cosolvents), which are regarded as nonionic surfactants with small polar head group [58,59] linear and aromatic alcohols are the most frequently used. Their role is twofold:

$\square$ to influence the phase behaviour and thus the stability of colloidal surfactant dispersions,

a or to be used as a fragrance material in chemical formulations.

\subsubsection{Effect on the Phase Behaviour}

It is of main interest to be able to determine the influence of cosurfactants on the physical properties of surfactants, in order to control the stability of various colloidal systems, which is directly related to the adsorption of surfactant at the water-oil interface, preventing in this manner the emulsion droplets from coalescing.

The phase behaviour, and hence the stability of surfactant solutions such as coarse emulsions and microemulsions is highly depended on three factors: the type, concentration and the chain length of used surface active molecules.

It has been already established [60] that the capability of the hydrocarbons for solubilization in the surfactant aggregates (formation of microheterogeneous, visually transparent aggregatively stable systems) increases in the series from paraffins to naphthenes to arenes; in each homologous series, the capability for being solubilized increase with increasing molecular weight of the hydrocarbon. It is known that arenes lower the degree of aggregation slightly and shift the region of the CMC.

If one uses ionic surfactants electrostatic interactions have to be screened by a proper component to stabilize the entire system. To avoid the unwanted effect of salt (salting out), it can be overcome by adding highly effective surface active molecules possessing an 
amphiphilic nature. Furthermore, most commercial ionic surfactants are not balanced with respect to their affinity to water and oil, which can be made so by addition of an alkyl chain alcohol as a cosurfactant, which interposes itself between surfactant molecules [61]. Basically, the alcohol head group, being nonionic serves to lessen the electrostatic interactions with the neighbouring charged surfactant head group which results in reduction of the charge density on aggregate micellar surface and the optimal area per head group [62-65].

Apparently, the introduction of ion-dipole interaction between the hydroxyl group of the alcohol and the surfactant head group causes a tighter packing of the micelle, resulting in a greater micellar stability [65]. However, the hydrocarbon tail of the alcohol contributes to the hydrophobic interaction, thus to the surfactant hydrocarbon volume. Such a local packing of cosurfactant and surfactant sets the spontaneous curvature of the interface, its flexibility and preferred size of noninteracting aggregates [66-69]. In this way the lower-curvature (smaller head-group area) structures are stabilized by added cosurfactant; e.g., the hexagonal phase gives way to the lamellar phase $[51,64]$.

The stability or slow micellar relaxation of pure surfactant micelles is a strong function of concentration [65]. At low micelle-water interface coverages of cosurfactant there is little influence on the adsorption of surfactant. In this case the electrostatics repulsion between the charged groups of surfactant is still important. At high micelle-water interface coverages, however, the average area per adsorbed surfactant molecule is much smaller than at low surface coverages, thus the adsorption of surfactant is significantly increased. This is explained by the fact that the positive interaction of the alkyl chains is increased, without simultaneously introducing additional repulsion trough negatively charged head groups. Therefore, the addition of a cosurfactant can stimulate the adsorption of surfactant only at rather high cosurfactant/surfactant rations [70].

Moreover, by changing alcohol concentration it is possible to modify the spontaneous curvature. For instance, in the case of the SDS/water/pentanol/dodecane quaternary system, increasing the alcohol concentration shifts the monolayer spontaneous curvature to more negative values, which leads to breakdown of the structure and formation of reverse micelles. Decreasing the alcohol concentration shifts the monolayer spontaneous curvature to more positive values, which results in sponge phase formation [66].

As linear alcohol molecules are aligned at interfaces, the properties of the interface are impacted to a large extent by the matching or mismatching of the alkyl chain lengths. In general, the chain length of surfactants used in a given system must be the same to maximize 
lateral molecular interactions that stabilize the surfactant film at the interface. However, the disruption in the molecular packing caused by chain length mismatching in a surfactant film leads to lower interaction energies and, hence, lower film stability relative to systems in which the chain lengths are equal [65]. It was shown earlier that chain length compatibility plays an important role not only at air/water or oil/water interfaces but also in micelles [71] and lipid bilayers.

As the lower alcohols are more soluble in water, their concentration can increase to a higher level and consequently the reduction of the $\mathrm{CMC}$ is increased further. The presence of higher alcohols, however, may decrease the polarity and hence the adsorption of cosurfactant may be increased leading to a higher stability $[72,73]$.

Another effect of the addition of the cosurfactant is that, when using short chain length alcohols, a decrease in micellar size is observed. On the other hand, using medium and long chain length alcohols increases the aggregation number producing larger alcohol-surfactant mixed micelles [74].

In microemulsions (see Subsection 2.1.4) and miniemulsions (a miniemulsion differs form the microemulsions only by the monomer size, which amounts to 0.05-0.2 $\mu \mathrm{m}$ ) [75] surface active additives are used to stabilise the emulsion droplets. In microemulsions mostly short chain alcohols (such as pentanol) are used as cosurfactant, whereas in miniemulsions medium and long chain alcohols (such as heptanol, octanol) are used.

The cosurfactants used change the adsorption behaviour of the surfactants at the water-oil interface. With the addition of a short-alkyl-chain alcohol the surfactant form the middle phase microemulsion, whereas a lamellar liquid crystal appears with a medium- or long-chain alcohol (heptanol, octanol, decanol) [76].

Lipid bilayers obey Traube's rule of interfacial tension reduction for short chain alcohols (methanol-butanol), i.e., for every additional $\mathrm{CH}_{2}$ group, an alcohol becomes three times more effective in decreasing the interfacial tension of the bilayer [77]. Therefore, the alcohol partitioning into the bilayer-water interface are responsible for the Traube's rule dependence, and hence for every additional $\mathrm{CH}_{2}$ group, three times more alcohol partitions into the interface.

Membrane permeability to these alcohols is also chain length dependent duo to more adsorption as the chain length increases. 
Summarizing the above facts: the type, concentration and chain length compatibility of surface active additives are important factors in systems involving interfacial surfactant films. Introduction of alkyl alcohol as cosurfactant has the following consequences:

a a decrease of the cmc;

a an increase of adsorption of surfactant at the interface;

口 an increase in interface flexibility;

a an increase or decrease of the micelle size;

口 a decrease in emulsion droplet size;

a an increase of the degree of dissociation of the micelles.

\subsubsection{Fragrances and Flavours}

"Fragrance and flavour substances are comparatively strong-smelling organic compounds with characteristic, usually pleasant odors. They are, therefore, used in perfumes and perfumed products, as well as for the flavouring of foods and beverages. Whether a particular product is called a fragrance or a flavour substance depends on whether it is used as a perfume or a flavour. Fragrances and flavours are, like taste substances, chemical messengers, their receptors being the olfactory cells in the nose" [78].

Perfume is thousands of years old - the word "perfume" comes from the Latin per fume "through smoke". One of the oldest uses of perfumes comes form the burning of incense and aromatic herbs used in religious services, often the aromatic gums, frankincense and myrrh, gathered from trees. The Egyptians were the first to incorporate perfume into their culture followed by the ancient Chinese, Hindus, Israelites, Carthaginians, Arabs, Greeks, and Romans.

In food, pharmaceutical and personal care products, the binding of fragrance and flavour materials to emulsions, microemulsions and polymers, especially proteins, has attracted considerable interest. New product formulations and significant fragrance levels made it crucial to understand the effect of fragrance on the surfactant systems, and the influence of the surfactant systems on the fragrance performance [79].

Besides aldehydes, ketones and esters, cosurfactants as linear and aromatic alcohols are classes of compounds that are represented most frequently in natural and artificial fragrances. The most successful fragrance is a simple rose, essentially phenylethyl alcohol $[79,80]$. 
Fragrances are added to formulated products for two main reasons:

- to mask the odour of the chemicals in the product;

- or to give the preparation an odour.

Important properties of fragrance chemicals include volatility, polarity, surface activity and stability. Some aroma chemicals can lead to the discoloration of a white emulsion or microemulsion, usually toward a brown or yellow hue. In extreme cases, the fragrance can also cause the emulsion or microemulsion to break. Therefore, the quality of the emulsion raw materials can decisively impact performance and stability. Another variable in emulsion bases is $\mathrm{pH}$; a fragrance may be fine in a lotion of $\mathrm{pH} 7$ but become unstable at the low $\mathrm{pH}$ of an AHA (alpha hydroxyl acid) treatment cream [79]. Moreover, fragrance can also trigger changes in viscosity, which become more pronounced as the fragrance level rises.

The typical use of the fragrance is the hydralcoholic application in perfumes, colognes or eau de toilettes. These systems, based on aqueous ethanol, are the least technically hostile of the environments where fragrance is used. The addition of methanol is sometimes done for cooling effect in aftershaves [79].

The majority of personal care products are either emulsions, such as creams and lotions, or surfactant systems, such as shampoos and bath gels. Examining the interaction of these two systems with fragrance reveals most of the essential issues that arise in fragrance application. Of course the fragrance can be considered as an additive, affecting on the CMC and many properties of surfactant dispersions as it is described in Section 2.2.1.

This study aims at the identification of the local environment of particular cosurfactants in various soft matter structures. As model guest molecules a series of phenyl alcohols with varying hydrocarbon chain lengths were used (denoted in the following by the number $n$ of carbons in the alkyl chain) as shown in Figure 9. 


$$
\begin{array}{lllll}
(n=2) & (n=3) \quad(n=4) \quad(n=5) & (n=2+3)
\end{array}
$$<smiles>OCCc1ccccc1</smiles>

$8.1 \AA$<smiles>OCCCc1ccccc1</smiles>

$9.3 \AA$<smiles>OCCCCc1ccccc1</smiles>

$10.1 \AA$<smiles>OCCCCCc1ccccc1</smiles>

$11.0 \AA$<smiles>CCCc1ccc(CCO)cc1</smiles>

$11.1 \AA$

Figure 9: Structure and chain length of phenyl alcohol guest molecules. The phenyl alcohols are distinguished by the number, $n$, of $\mathrm{CH}_{2}$ groups in their hydrocarbon chain: 2-phenylethan1-ol $(n=2)$, 3-phenylpropan-1-ol $(n=3)$, 4-phenylbutan-1-ol $(n=4)$, 5-phenylpentan-1-ol $(n$ $=5)$ and p-propyl-2-phenylethan-1-ol $(n=2+3)$. It is anticipated that the alcohol resides in water or in the bilayer with the HO-group at the surfactant-water interface. Probing of the environment occurs by a fully polarised muon spin label that is introduced by addition of the muonium atom to the phenyl ring which dips into the bilayer to different depths. The length of the fully stretched-out guest molecule is given in $\AA$.

\subsection{Muon Spin Resonance}

\subsubsection{The Positive Muon, Muonium and Muoniated Radicals}

The muon $(\mu)$ which was discovered by Neddermeyer and Anderson in 1937, is an elementary particle with a spin of $1 / 2$, a lifetime of $2.197 \mu \mathrm{s}$, a magnetic moment 3.18 times that of the proton and a mass approximately one-ninth that of the proton. The muon exists in two charge states, as $\mu^{-}$and $\mu^{+}$. A negative muon can replace an electron in an atom, and due to its huge mass, is captured by nuclei forming muonic atoms. The $\mu^{-}$lifetime is reduced by the reaction with the nucleus and the spin polarization is partly lost during the cascade into the ground electronic state, so the negative muon is not used as widely as the positive muon, which does not react with nuclei in matter because of the repulsive Coulomb interaction. 
The polarisation of the positive muon is almost fully conserved when stopped in matter. A fraction of the implanted muons will end up in diamagnetic chemical environments; as 'bare' muons, solvated muons or substituted for the proton of a diamagnetic molecule. It is not possible to obtain the necessary spectral resolution to distinguish between muons in different diamagnetic environments because of the short lifetime of the muon. Another fraction of implanted muons can pick up an electron during the radiolysis process to form a one-electron atom called muonium $\left(\mathrm{Mu}=\left[\mu^{+}, e^{-}\right]\right)$that was detected in 1957 for the first time. Even though the mass of the muon is much less than that of the proton, it is still about 200 times more massive than that of the electron, so the reduced masses of $\mathrm{H}$ and $\mathrm{Mu}$ are almost the same. This means that their chemical properties are also almost the same, and $\mathrm{Mu}$ can be considered to be an isotope of $\mathrm{H}$, just like $\mathrm{D}$ or $\mathrm{T}$ [81]. The relative fraction of diamagnetic and paramagnetic states depends on the chemical properties of the medium.

Muonium can add to unsaturated bonds of organic molecules to produce an organic radical (called a muoniated radical), with the muon acting as a spin label [17]. Several hundred muoniated radicals have been identified in the solid, liquid and gas phases. The muoniated cyclohexadienyl radical is produced by the reaction of $\mathrm{Mu}$ with benzene [82] (see Figure 10).

The muoniated radical differs from benzene only by an additional hydrogen atom, which in the muoniated form is the light hydrogen isotope muonium. Such spin labelled tracer radicals have recently been used as probes of soft condensed matter [6,83-86].

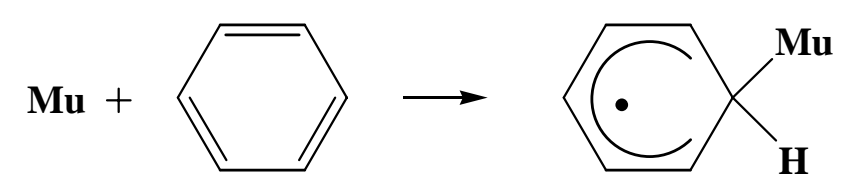

Figure 10: Formation of the muoniated cyclohehaxadienyl radical derived form benzene. Adopted from [83].

In the present work the spin labelling is achieved by muonium addition to the unsaturated bonds of the phenyl ring in the ortho, meta and para positions with respect to the hydroxyalkane chain of the series of phenyl alcohols $\left(\mathrm{C}_{6} \mathrm{H}_{5}\left(\mathrm{CH}_{2}\right)_{n} \mathrm{OH}\right.$ with $2 \leq \mathrm{n} \leq 5$, and $\left.\mathrm{CH}_{3}\left(\mathrm{CH}_{2}\right)_{2} \mathrm{C}_{6} \mathrm{H}_{4}\left(\mathrm{CH}_{2}\right)_{2} \mathrm{OH}\right)$ placing the muon into cyclohexadienyl type radicals.

Any changes in geometry, spatial requirements, polarity, and polarizability of the tracer molecules on $\mathrm{Mu}$ addition to the aromatic ring are small. Hence, the muon spin label is 
considered a nearly nonperturbing probe [84]. Such muoniated radicals act as spectators of their local environments, in particular of the polarity, and of the changes in the properties of the colloidal systems. Here, it permits the investigation of the partitioning, reorientational dynamics and solvation thermodynamic properties of five different phenyl alcohols in a cationic surfactant emulsion (DHTAC), and anionic surfactant dispersions (SDS).

\subsubsection{The ALC- $\mu$ SR Technique}

"Muons occur in nature as a result of several decays in cosmic rays. More specifically, the nuclei that make up cosmic rays are able to travel from their distant sources to the Earth because of the low density of matter in space. Nuclei interact strongly with other matter, so when the cosmic rays approach Earth they begin to collide with the nuclei of atmospheric gases. These collisions, in a process known as a shower, result in the production of many pions and kaons, unstable mesons that quickly decay into muons. Because muons do not interact strongly with the atmosphere and because of the relativistic effect of time dilation many of these muons are able to reach the surface of the Earth. Muons are ionizing radiation, and may easily be detected by many types of particle detectors such as bubble chambers or scintillation detectors. If several muons are observed by separated detectors at the same instant it is clear that they must have been produced in the same shower event" [87].

Under laboratory conditions, however, the muon is produced at suitable accelerators. As is presented in Figure 11 the accelerator is the "Ring", a separated sector cyclotron providing a high power proton beam at an energy of $590 \mathrm{MeV}$.

Positive muons are produced by the decay of positive pions with a lifetime $26 \mathrm{~ns}$, which are in turn produced by the reaction of high-energy protons with a graphite or beryllium target with a length of $60 \mathrm{~mm}$.

$$
\pi^{+} \rightarrow \mu^{+}+v_{\mu}
$$

Beams of monoenergetic muons are available in which the spin polarization is close to $100 \%$. The muons originating from decay of pions stopped at the surface of the target are so-called surface muons with a momentum of $28 \mathrm{Me} / \mathrm{c}$ and a stop range $140 \mathrm{mg} \mathrm{cm}{ }^{-2}$, which corresponds to a water layer of $1.4 \mathrm{~mm}$ thickness. Hence, the surface muons allow working with very thin samples. Their implantation into matter leads to the formation of the muonium atom (Mu). 


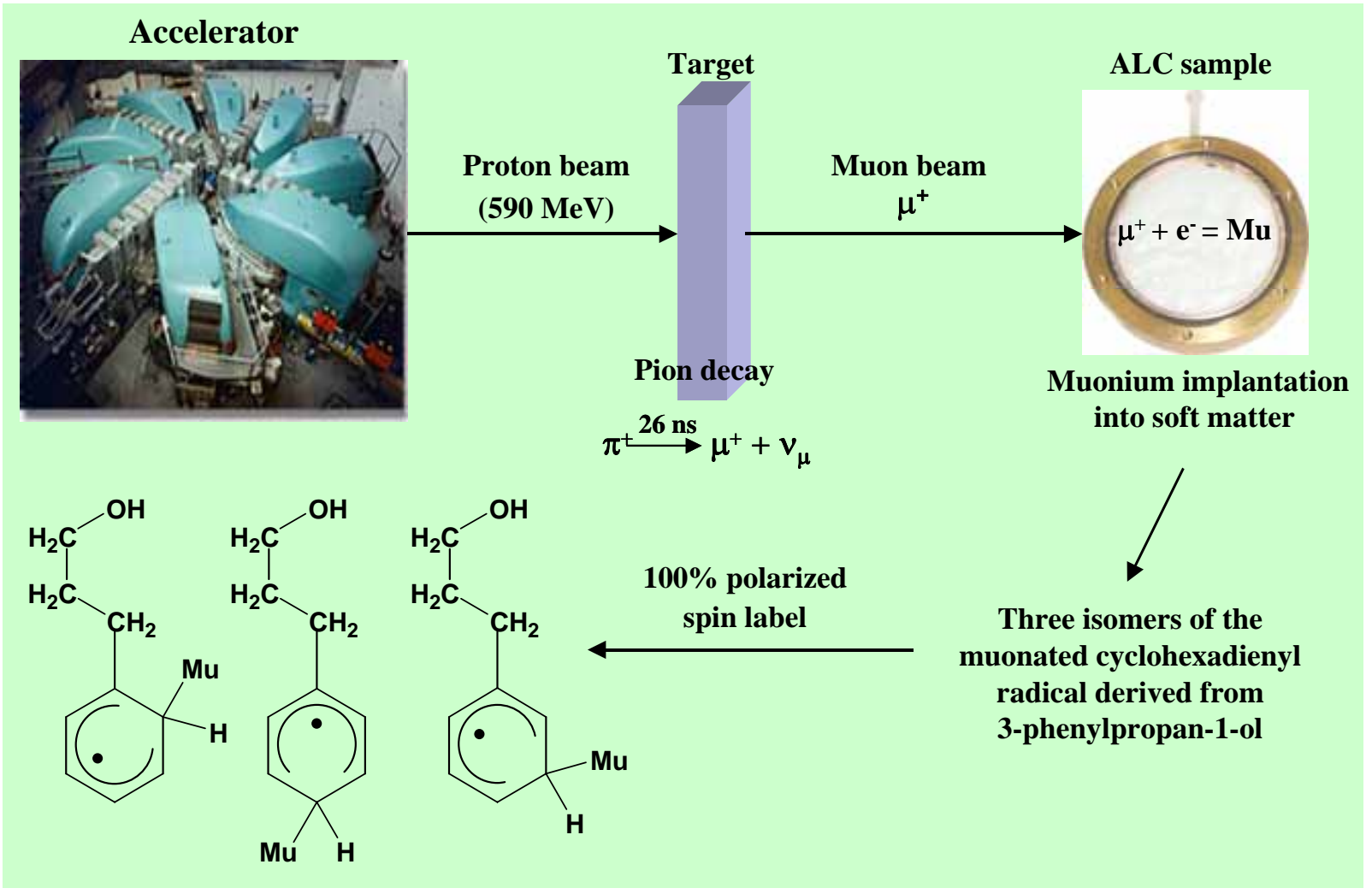

Figure 11: General scheme of the production of the positive muon, muonium and muoniated cyclohexadienyl radical derived from $\mathrm{C}_{6} \mathrm{H}_{5}\left(\mathrm{CH}_{2}\right)_{3} \mathrm{OH}$ in the ALC- $\mu \mathrm{SR}$.

Muonium addition to the phenyl ring in the ortho, para and meta positions to the hydroxyalkane chain places the muon into the cyclohexadienyl radical derived from 3-phenylpropan-1-ol $(n=3)$ as in shown in Fig. 11. This procedure is common for all phenyl alcohols, which were used in this experiment.

The muon decay, which violates parity, produces a positron $\left(e^{+}\right)$and two neutrinos $\left(v_{e}\right.$ and $\left.v_{\mu}\right)$.

$$
\mu^{+} \rightarrow e^{+}+v_{e}+v_{\mu}
$$

The violation of parity causes the positron to be emitted preferentially along the direction of the muon spin, and the detection of the decay positrons as a function of time provides a convenient means of monitoring the temporal evolution of the muon spin.

Summarizing the above facts, the combination of high spin polarisation, anisotropic decay and single particle counting detection makes the muon an exceptionally sensitive probe of magnetic phenomena [16].

Several variants of experimental $\mu$ SR techniques have been developed in order to adapt to particular problems. 
a)

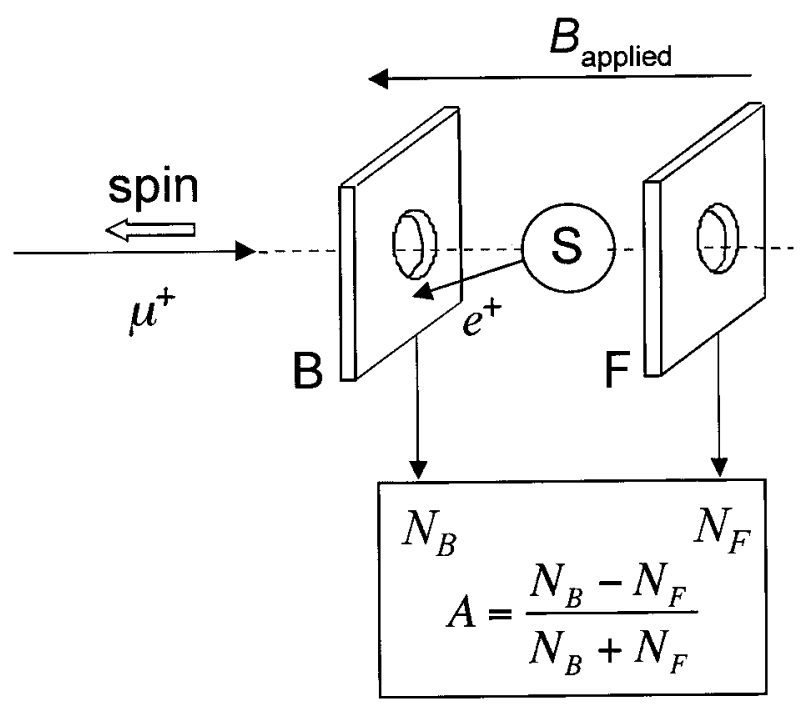

b)

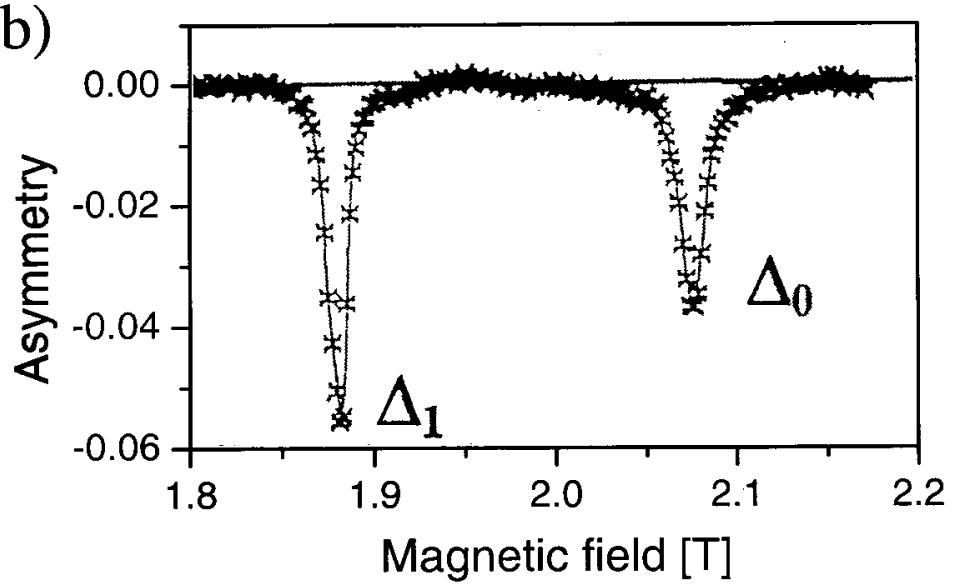

Figure 12: (a) Schematic drawing of the experimental setup for the ALC- $\mu$ SR experiment, (b) ALC- $\mu$ SR spectrum of the $\mathrm{C}_{6} \mathrm{H}_{6} \mathrm{Mu}$ radical obtained with benzene adsorbed on siliceous ZSM5 zeolite in the region of the $\Delta_{1}$ and $\Delta_{0}$ methylene proton resonances. Note that the $\Delta_{1}$ represents the static case in the absence of any dynamic averaging is of much higher intensity than the $\Delta_{0}$ line. Figure adopted from [83].

ALC- $\mu$ SR experiments presented in this work were performed in a longitudinal magnetic field with respect to the initial muon spin polarisation, which makes it possible to study systems with low target molecule concentrations, down to a few $\mathrm{mM}$ for substituted aromatics. In the ALC- $\mu$ SR measurements, the number of decay positrons emitted in the forward and backward directions with respect to the momentum of the incoming muon beam $\left(N_{F}\right.$ and $N_{B}$, respectively) are measured as a function of the applied magnetic field. The asymmetry (timeintegrated longitudinal muon spin polarisation) is given by the normalized difference of counts, $\left(N_{B}-N_{F}\right) /\left(N_{B}+N_{F}\right)$. The ALC- $\mu$ SR technique has been extensively reviewed $[88,89]$. The setup for an ALC- $\mu$ SR experiment and a sample spectrum are shown in Figure 12. 
Samples for ALC- $\mu$ SR experiments were always kept in brass containers fixed on copper plates connected to the cryostat to enable rapid thermal equilibrium. The thickness of the sample itself has to be sufficient so that the muons are stopped inside in the sample compound and cannot pass the cell. It of course depends on the density of chemical substances and the energy of the incoming muons. ALC cells are appropriate for solid and liquid compounds. In addition, all measurements were performed under vacuum to avoid spin exchange with oxygen molecules.

In high magnetic fields, the eigenstates of a muoniated radical are pure Zeeman states so there is no evolution of the muon spin with time and the asymmetry is independent of the applied field. At specific values of the applied field, nearly degenerate pairs of spin states can be mixed through the isotropic and anisotropic components of the hyperfine interaction. The muon polarisation oscillates between the two mixing states of the avoided crossing. This results in a resonant-like change in the asymmetry as the magnetic field is swept. There are three types of resonances, which are characterised by the selection rule $|\Delta M|=0,1$ and 2 , where $M$ is the sum of the quantum numbers for the z-components of the muon, electron and nuclear spins (see Figure 13).

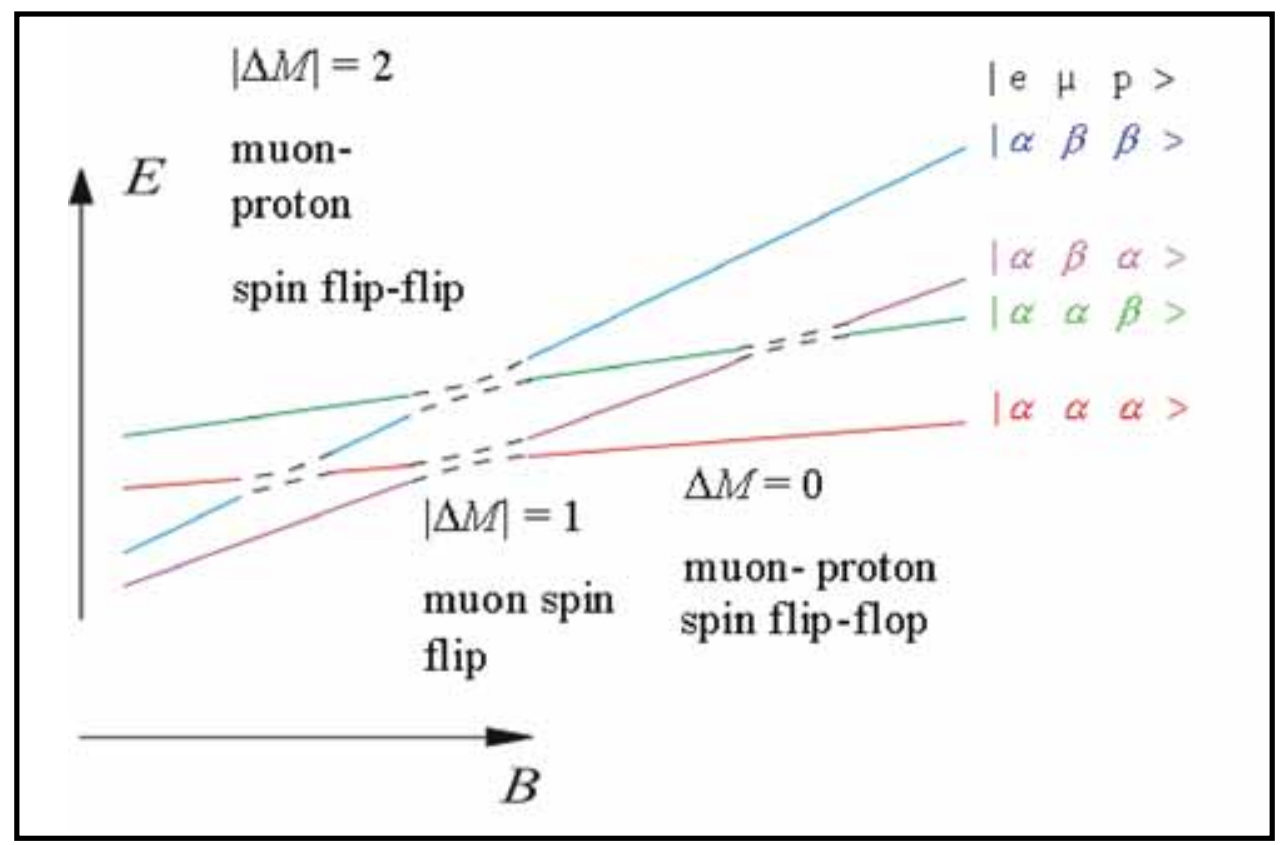

Figure 13: High-field energy-level diagram for a three spin system with electron $(e)$, muon $(\mu)$ and proton $(p)$. Dotted lines refer to avoided level crossings. 
They occur within the same electron spin subsystem and are subsequently denoted in abbreviated form as $\Delta_{0}, \Delta_{1}$ and $\Delta_{2}$ transitions. The resonance fields are given by:

$$
B_{r}=\left|\frac{A^{\mu}(\theta, \varphi)+(|\Delta M|-1)\left[A^{k}(\theta, \varphi)\right]}{2\left[\gamma_{\mu}+(|\Delta M|-1) \gamma_{\mathrm{k}}\right]}\right|
$$

where $A^{\mathrm{\mu}}(\theta, \varphi)$ and $A^{\mathrm{k}}(\theta, \varphi)$ are the effective muon and nuclear hyperfine coupling constants (hfcc) in units of MHz (in general $\boldsymbol{A}^{\mathbf{\mu}}$ and $\boldsymbol{A}^{\mathbf{k}}$ are tensors, each of which is a sum of an isotropic component due to the Fermi contact interaction and an anisotropic component due to direct dipolar coupling which in the following is denoted $\boldsymbol{D}^{\boldsymbol{\mu}}$ and $\boldsymbol{D}^{\mathbf{k}}$, therefore the effective hfcc depend on orientation), and $\gamma_{\mu}$ and $\gamma_{\mathrm{k}}$ are the corresponding gyromagnetic ratios $\left(\gamma_{\mu}=\right.$ 135.50 $\mathrm{MHz} \mathrm{T}^{-1}, \gamma_{\mathrm{k}}=42.580 \mathrm{MHz} \mathrm{T}^{-1}$ for protons).

The $\Delta_{0}$ resonance is due to transitions between spin states that have opposite muon and nuclear spins, mixed by the isotropic hyperfine interaction (muon-nuclear spin flip-flop transition) and are observed for muoniated radicals in solids, liquids or gases. This type of resonance is used to measure nuclear hfccs, allowing a full characterisation of radicals with known muon hfcc.

The $\Delta_{1}$ resonances arise from transitions between spin states that have the same electron and nuclear spins but different muon spin (muon spin flip transition). These spin states are only mixed in the presence of anisotropy, so the presence of a $\Delta_{1}$ resonance can be considered to be diagnostic of a frozen state or of anisotropic motion.

Summarizing the above facts, the position and the area of the $\Delta_{0}$ resonance, as well as the shape of the $\Delta_{1}$ resonance provide detailed information about the sample compound.

The resonances corresponding to the magnetic selection rule $\Delta M=0\left(\Delta_{0}\right)$ represent the exchange of spin polarization between the muon and a magnetic nucleus. To first order they occur at fields:

$$
B_{r}\left(\Delta_{0}\right)=\frac{A^{\mu}-A^{k}}{2\left(\gamma_{\mu}-\gamma_{k}\right)}-\frac{A^{\mu}+A^{k}}{2 \gamma_{e}}
$$

A different type of resonance with $\Delta M=1\left(\Delta_{1}\right)$ can be calculated from:

$$
B_{r}\left(\Delta_{1}\right)=\frac{A^{\mu}}{2 \gamma_{\mu}}-\frac{A^{\mu}}{2 \gamma_{e}}
$$

ALC muon spin resonance was used here to elucidate the affinity of five different phenyl alcohols for the aqueous and nonaqueous environments in the lamellar phase of a 
DHTAC/water binary system as well as the partitioning dependence on temperature and on chain length defined by $n\left(\mathrm{CH}_{2}\right)$.

Moreover, ALC- $\mu \mathrm{SR}$ permitted the determination of the Heisenberg spin exchange rate constant between the $\mathrm{Mu}$ adducts of 2-phenylethanol $(n=2)$ and paramagnetic $\mathrm{Ni}^{2+}$ ions from the broadening of the $\Delta_{0}$ resonances, which provides a second means for identifying the preferred location of the muoniated radical in lamellar phase dispersions composed of the dichain cationic surfactant.

To find out whether the intercalation of the phenylalcohol probe molecules depends on the curvature of the water-surfactant interface, ALC- $\mu$ SR was applied to monitor the location and the dynamics of the muoniated 3-phenylpropan-1-ol $(n=3)$ and 5-phenylpentan-1-ol $(n=5)$ molecules in more complex environments as micelles, lamellar, rectangular, hexagonal and sponge phase of the SDS/water/pentan-1-ol ternary mixtures and the SDS/water/pentan-1ol/dodecane quaternary systems.

\subsubsection{The Effect of Anisotropy}

The principal axes of two hyperfine tensors $\boldsymbol{A}^{\boldsymbol{\mu}}$ and $\boldsymbol{A}^{\mathbf{k}}$ have an orientation relative to each other that depends on the geometry and electronic structure of the radical. Furthermore, at a given time the radical itself assumes a certain orientation with respect to the external field. For a single crystal with a single orientation of the radical we expect resonances of Lorentzian shape at a resonance field given by one set of effective hfcc according to eqn. (2-7). In ideal polycrystalline samples we have all possible crystal orientations with respect to the field, so that the resonances are superpositions of single crystal spectra with orientation dependent resonance fields, and their envelopes are called 'powder patterns' [90].

The resonances are suitable for studying anisotropic reorientational motion of radicals in single crystals as well as in polycrystalline or amorphous states because of the orientation dependence of the effective hfcc. In particular the line shape of the $\Delta_{1}$ resonance is extremely sensitive to, and characteristic of, the type of reorientational motion that the muoniated radical is undergoing. Figure 14 shows the simulated spectra of the muoniated cyclohexadienyl radical around the $\Delta_{1}$ and the $\Delta_{0}$ methylene proton resonances for different types of reorientational motion. The top spectrum gives the static powder pattern; the others are for 
fast uniaxial rotation about specific axes. We see that the lines are narrower than for the static case, and they assume a characteristic axial shape.

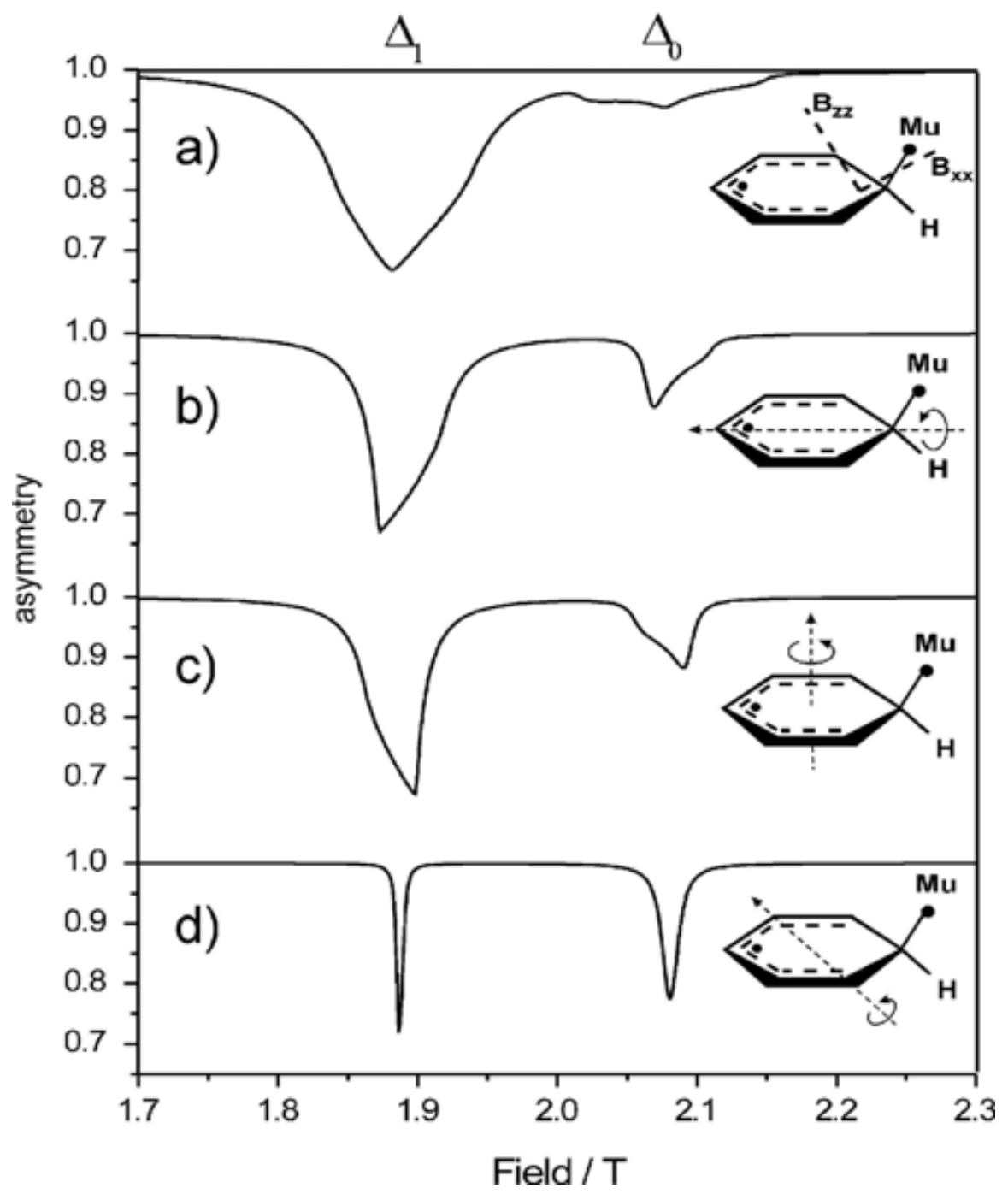

Figure 14: Simulated ALC- $\mu$ SR spectra of the $\mathrm{C}_{6} \mathrm{H}_{6} \mathrm{Mu}$ radical (a) for the fully static case, (b) for fast rotation about an axis in plane through the methylene CHMu group, (c) for fast rotation about an axis perpendicular to the molecular plane, and (d) for fast rotation about an axis through an ortho carbon atom and the centre of the phenyl ring.

The rotational axis becomes the new symmetry axis of the hyperfine tensors, and it is common to both $A^{\mathbf{\mu}}(\theta)$ and $A^{\mathbf{k}}(\theta)$. There is no longer any $\varphi$ dependence, and $\theta$ is the angle with respect to the external field. There exists a specific orientation of the rotation axis along which the component of the muon hyperfine tensor is equal to its isotropic value; rotation about this axis averages out all anisotropy, and the $\Delta_{1}$ resonance gets narrower and finally disappears. Obviously, the last entry of Figure 14 is close to this case; a further reduction of 
the muon hyperfine anisotropy would lead to an intensity reduction of the $\Delta_{1}$ resonance and finally to its disappearance.

An axial line shape with a further reduced width would indicate a more extensive averaging by tumbling or wobbling motion of the rotation axis. The effect can be simulated by a Monte Carlo technique, assuming reorientational jumps according to a given model and with a certain correlation time $\tau_{\mathrm{c}}$ [91]. Analyses of the line shapes of the resonances in the ALC- $\mu$ SR spectra arising from $\mathrm{Mu}$ adducts of norbornene, $\mathrm{C}_{60}$ and $\mathrm{C}_{70}$ have provided valuable information about the dynamics of these radicals [88].

The $\Delta_{1}$ resonances are averaged out fully by isotropic motion on a critical time scale given by the inverse of the hyperfine anisotropy (typically $50 \mathrm{~ns}$ for cyclohexadienyl type radicals). The $\Delta_{2}$ resonance is also specific to anisotropic environments, but it is very weak and narrow and therefore has not been used for quantitative studies.

Of utmost importance for the interpretation of the present experimental spectra in terms of dynamics are the widths and the relative amplitudes of the $\Delta_{1}$ and the $\Delta_{0}$ methylene proton resonances.

The two types of resonances are of different origin, based on different selection rules, and have different interpretations: under the conditions relevant to the present experiments the areas of the $\Delta_{0}$ resonance lines are proportional to the yields of the corresponding species, while the $\Delta_{1}$ resonances are indicators of the effective muon hyperfine anisotropy so that their principal proportionality to the yield is only relevant under comparable dynamic conditions (for example in the static case). The absence of the $\Delta_{1}$ line reveals fast reorientational dynamics in isotropic environments (or rotational motion about a very special axis). The presence of $\Delta_{1}$ lines with lower amplitudes than $\Delta_{0}$ lines indicates that the radical is in an anisotropic environment and undergoes quite extensive dynamic averaging of the hyperfine anisotropy.

Polarized positive muons substituted for hydrogen nuclei in muoniated radicals derived from the 3-phenylpropan-1-ol $(n=3)$ were used in this case as spin labels to sense reorientational motion of the radical in the environment of DHTAC and SDS surfactant systems. 


\section{Chapter 3}

\section{EXPERIMENTAL}

This Chapter is concerned with experimental details. It includes the description of used chemicals and sample preparation in the first Section. Determination of the phenyl alcohol solubility by UV-Vis is described in the second Section, while the density measurements of $p$ propyl-2-phenylethan-1-ol are discussed in Section 3.3. In order to define the temperature of the $\mathrm{L}_{\beta} / \mathrm{L}_{\alpha}$ phase transition of the DHTAC as well as the stability of the SDS mixtures the differential scanning calorimetry, DSC was applied (see Section 3.4). However, the polarized optical microscopy, POM (Section 3.5) was used to observe textures of the SDS based mesophases. The last Section of this Chapter contains the description of the experimental setup and of the data analysis of the ALC- $\mu$ SR technique.

\subsection{Sample Preparation}

Triply distilled water used in all experiments was prepared at the University of Stuttgart. The commercial components were used without further purification.

\subsubsection{DHTAC Dispersions Including Cosurfactants}

The cationic surfactant, 2,3-diheptadecyl ester ethoxypropyl-1,1,1-trimethylammonium chloride (DHTAC) at a purity of 94\% was obtained in recrystallised form from Unilever Research Port Sunlight. The phenyl alcohol cosurfactants were purchased at high purity (>98\%) from Sigma-Aldrich, except for $p$-propyl-2-phenylethan-1-ol that was synthesized by Tobias Burkert, University of Stuttgart (98.7\% purity confirmed by gas chromatography) [92] $n$-octadecane was purchased from Merck at a purity of $>99 \%$. 
Surfactant, phenyl alcohols, water and $n$-octadecane were first deoxygenated by bubbling dry nitrogen gas through the liquid (surfactant and $n$-octadecane in their molten state) for approximately 1 h. $15 \mathrm{wt} \%$ dispersions of the DHTAC simple lamellar phase were prepared in deoxygenated water by melting the surfactant and shear mixing the melt through a narrow orifice. $40 \mathrm{mM}$ phenylethan-1-ol was cold-mixed under oxygen-free conditions by shearing through a narrow orifice with $15 \%$ surfactant dispersion, or $n$-octadecane, or water. The other phenyl alcohols at concentrations of $4-40 \mathrm{mM}$ were hot-mixed in the same manner as described above.

\subsubsection{Nickel Samples for Heisenberg Spin Exchange Experiments}

Nickel(II) chloride hexahydrate was obtained from Sigma-Aldrich at 99.9999\% purity.

Two series of solutions in an oxygen free environment were prepared for this experiment. The first consisting of $40 \mathrm{mM}$ 2-phenylethan-1-ol and nickel chloride at 0, 1, 2, 3 and $5 \mathrm{mM}$ concentrations in bulk water in the absence of detergent, and the second consisting of the same alcohol and $\mathrm{Ni}^{2+}$ at the same concentrations in 15\% aqueous DHTAC emulsions. The sample preparation was carried out under oxygen-free conditions in a glove box and all substances were degassed prior to use.

\subsubsection{SDS Dispersions Including Cosurfactants}

The anionic surfactant, sodium $n$-dodecyl sulfate (SDS), was obtained from Calbiochem, Canada, at a purity of $99.8 \%$ and did not contain any $n$-dodecanol as confirmed by gas chromatography. 1-Pentanol and $n$-dodecane were purchased from Sigma-Aldrich at a purity of $>99 \%$.

The studied lyotropic samples are ternary mixtures composed of SDS, water and 1pentanol, and quaternary systems consisting of SDS, water, 1-pentanol and $n$-dodecane. All examined mesophases had a constant water/SDS weight ratio of $1.552 \pm 0.002$, and the compositions are given in Table 5. Phenyl alcohols, water, 1-pentanol and $n$-dodecane were first bubbled with dry nitrogen gas for approximately one hour to make them oxygen-free, and $10 \mathrm{mM}$ samples of 3-phenylpropan-1-ol $(n=3)$ or of $5 \mathrm{mM}$-phenylpentan-1-ol $(n=5)$ were 
then prepared in a glove box at room temperature by gentle mixing with the previously prepared particular SDS mesophases, $n$-dodecane and water, respectively.

Table 5: Compositions of the ternary and quaternary SDS samples with a constant weight ratio water/SDS of $1.552 \pm 0.002$.

\begin{tabular}{|c|c|c|c|c|c|}
\hline Phase $^{\mathrm{a}}$ & $\begin{array}{c}\text { SDS } \\
\%\end{array}$ & $\begin{array}{c}\text { water } \\
\% \\
\end{array}$ & $\begin{array}{c}1-p e n t a n o l \\
\%\end{array}$ & $\begin{array}{c}n \text {-dodecane } \\
\% \\
\end{array}$ & reference \\
\hline normal micelles $\mathrm{L}_{1}^{1}$ & 19.6 & 30.4 & 50 & 0 & this work \\
\hline microemulsion $\mu \mathrm{E}^{2}$ & 12.15 & 18.85 & 50 & 19 & this work \\
\hline microemulsion $\mu \mathrm{E}^{3}$ & 3.92 & 6.08 & 40 & 50 & this work \\
\hline lamellar $\mathrm{D}^{4}$ & 29.4 & 45.6 & 25 & 0 & this work \\
\hline reverse lamellar $\mathrm{D}^{5}$ & 15.1 & 23.35 & 14.55 & 47 & Hashimoto [93] \\
\hline reverse sponge $\mathrm{L}_{3}{ }^{6}$ & 11.65 & 18.05 & 10.3 & 60 & Lei [94] \\
\hline reverse sponge $\mathrm{L}_{3}{ }^{7}$ & 5.55 & 8.6 & 8.95 & 76.9 & Lei [94] \\
\hline rectangular $\mathrm{A}^{8}$ & 35.3 & 54.7 & 10 & 0 & this work \\
\hline rectangular $\mathrm{A}^{9}$ & 31.37 & 48.63 & 10 & 10 & this work \\
\hline hexagonal $\mathrm{E}^{10}$ & 36.1 & 56.12 & 7.78 & 0 & Koltover [95] \\
\hline reverse hexagonal $\mathrm{F}^{11}$ & 20 & 31 & 9 & 40 & this work \\
\hline
\end{tabular}

a) The superscript indicates the composition index as given in Figure 33 (see Subsection 4.2.1).

The resulting dispersions described in Subsections 3.1.1 to 3.1.3 were loaded into brass cells with $17 \mathrm{ml}$ internal volume (Figure 15), sealed with Viton o-ring to avoid leakage, and closed with a $50 \mu \mathrm{m}$ brass foil.

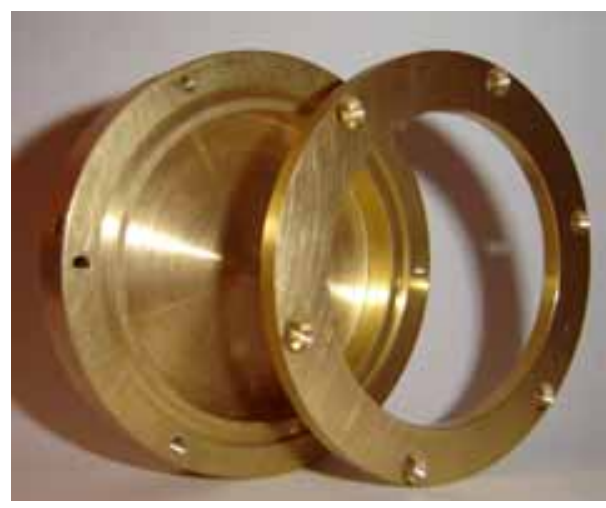

Figure 15: Brass sample cell for ALC- $\mu$ SR measurements. 


\subsection{Determination of Phenyl Alcohol Solubility in Water}

A Perkin-Elmer Lambda2 UV-Vis spectrometer was used to determine the solubility in water of all alcohols except 2-phenylethan-1-ol. Aqueous phenyl alcohol solutions were prepared in nominal 2, 3, 4, 6 and $30 \mathrm{mM}$ concentration and stirred for $5 \mathrm{~min}$ at $70{ }^{\circ} \mathrm{C}$. They were then stored in the glass flasks in the cabinet dryer for $48 \mathrm{~h}$ at $25^{\circ} \mathrm{C}$ before measurements to permit phase separation in case of supersaturated solutions. The absorbance and the molar absorptivity coefficient were measured at the wavelength of an absorption maximum. At saturation, the absorbance no longer increased with added nominal concentration.

\subsection{Density Measurements}

The density of $p$-propyl-2-phenylethan-1-ol was measured using a pycnometer (Brand, Germany) with a volume of $5.379 \mathrm{~cm}^{3}$ and calibrated for $20^{\circ} \mathrm{C}$.

\subsection{Differential Scanning Calorimetry}

The $\mathrm{L}_{\beta} / \mathrm{L}_{\alpha}$ phase transition of the DHTAC surfactant dispersions without and containing phenyl alcohols was measured by a DSC 204 differential scanning calorimeter (NETZSCH, Germany). All solutions were injected into aluminium pans, closed hermetically and heated in a temperature range $10-75{ }^{\circ} \mathrm{C}$.

Using the same method the stability of the SDS mixtures comprising the phenyl alcohols was determined. All solutions were also injected into aluminium pans, closed hermetically and heated this time in a temperature range from -20 to $+80{ }^{\circ} \mathrm{C}$.

In the case of both surfactant dispersions thermograms were recorded on heating and cooling at a rate of $5{ }^{\circ} \mathrm{C} \mathrm{min}^{-1}$. The system was calibrated using the following standards: $\mathrm{KNO}_{3}$, In, $\mathrm{Bi}, \mathrm{Sn}, \mathrm{Zn}, \mathrm{CsCl}, \mathrm{Hg}$ and $\mathrm{C}_{6} \mathrm{H}_{12}$. 


\subsection{Polarized Optical Microscopy}

For texture observations the SDS based mesophases containing the phenyl alcohols were placed between standard glass slides and measured in a Leica DMLP optical microscope at room temperature. Textures were photographed using a Nikon Digital camera.

\subsection{Avoided Level Crossing Muon Spin Resonance}

\subsubsection{The Experimental Setup}

The ALC- $\mu \mathrm{SR}$ experiments were carried out using positive muons at the ALC spectrometer at beam line $\pi \mathrm{E} 3$ (see Figure 16) of the Swiss Muon Source, Paul Scherrer Institut, Villigen, Switzerland. The ALC sample was placed in the centre of the magnet bore.

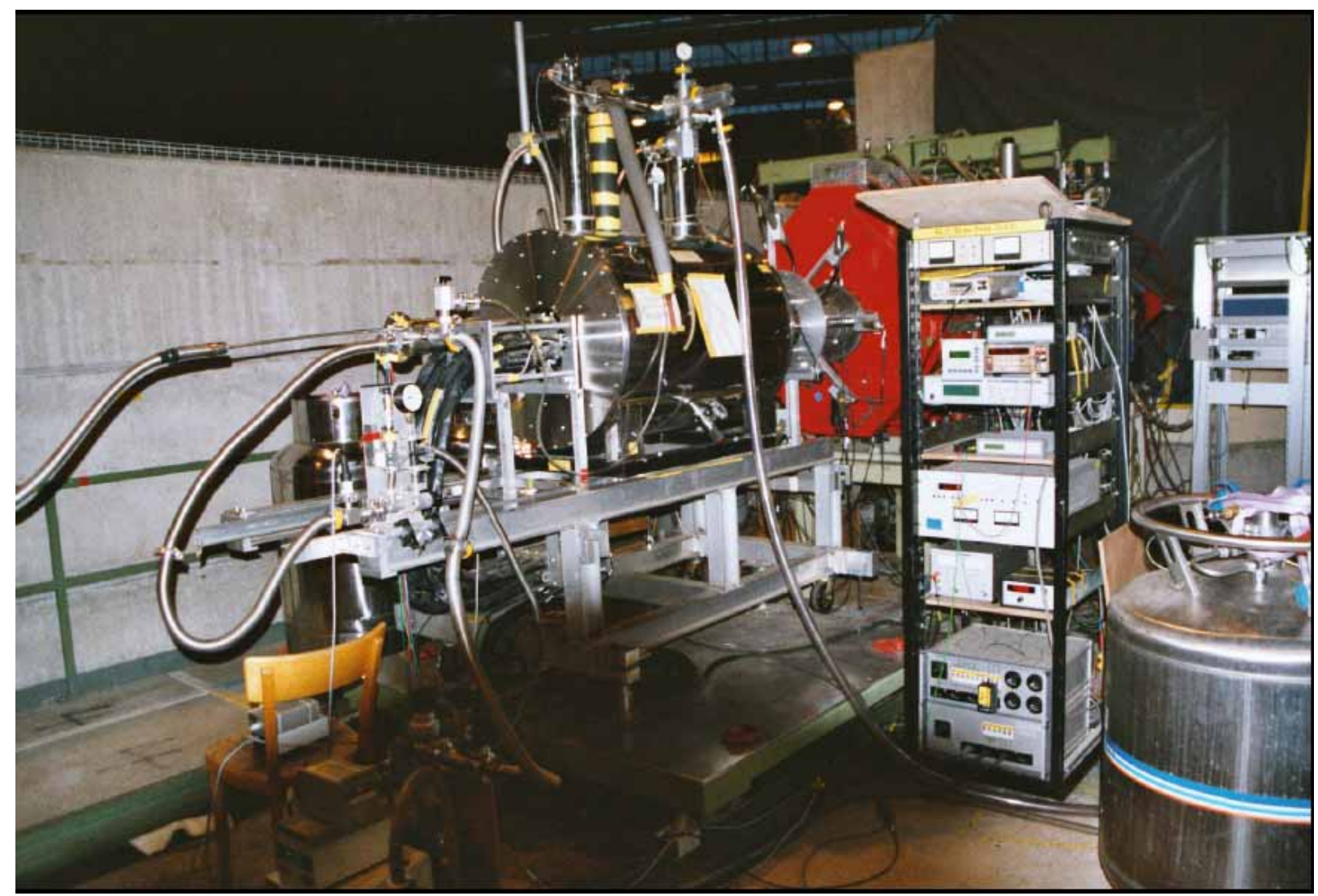

Figure 16: ALC spectrometer with 5 Tesla solenoid. 
ALC spectra were recorded over the field range 1.7-2.2 $\mathrm{T}$ using a superconducting solenoid at following temperatures:

a) from 25 to $75^{\circ} \mathrm{C}$ for the DHTAC system;

b) at $25,55,75^{\circ} \mathrm{C}$ for the spin exchange experiments;

c) at $25{ }^{\circ} \mathrm{C}$ for the SDS system.

One heating-cooling cycle on a $4 \mathrm{mM} p$-propyl-phenylethan-1-ol $(n=2+3)$ in the DHTAC emulsion was done to prove equilibrium of the system.

\subsubsection{Data Analysis}

\section{Fitting Procedure}

A representative example of raw data obtained from ALC- $\mu$ SR experiments without any “manipulation” is presented in Figure 17.

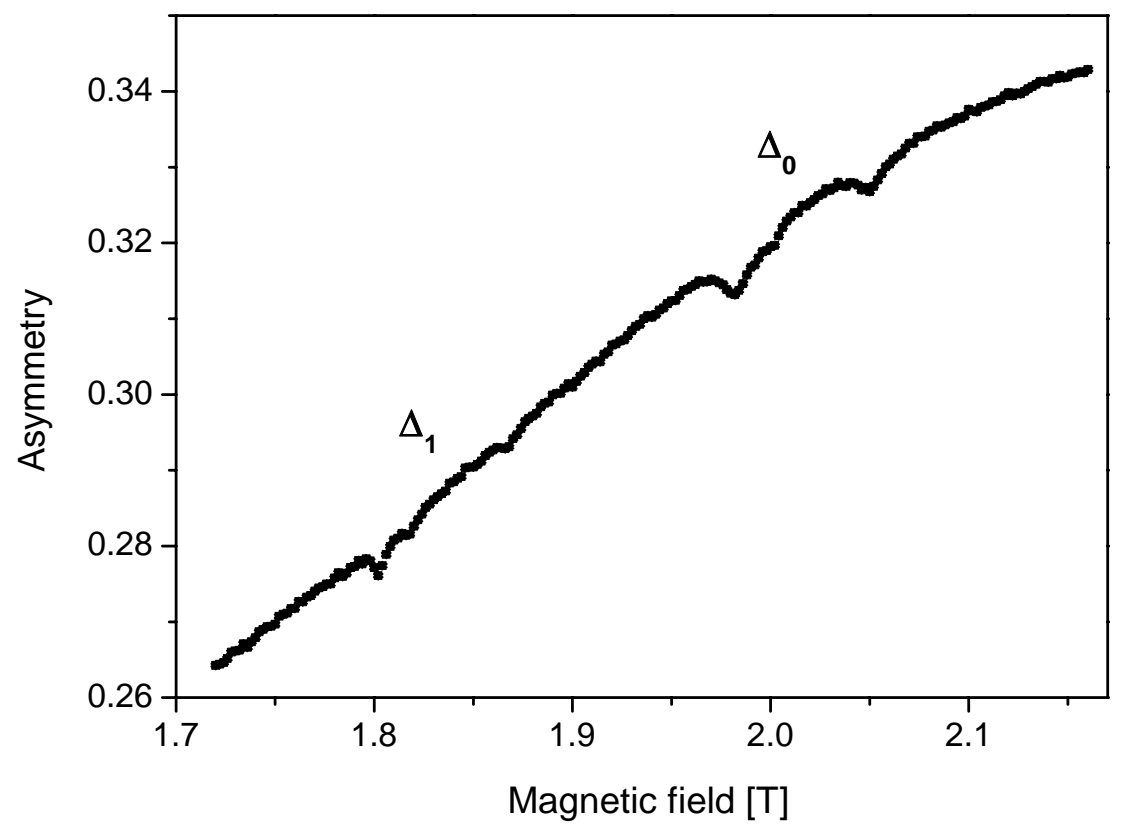

Figure 17: ALC- $\mu$ SR spectrum of $18 \mathrm{mM}$ 3-phenylpropan-1-ol in 15\% aq. DHTAC emulsion at $75^{\circ} \mathrm{C}$ before subtraction background.

As shown in Figure 18 the raw data were corrected by subtracting the background (measured on pure water) and analysed by fitting multiple Lorentzians to the experimental data using two different procedures. 
One of them was based on the MINUIT function minimization library [96] in order to determine:

a a \% aqueous character (see Subsection 4.1.2.1);

口 or a spin exchange rate constant $k$ (see Subsection 4.1.4.1).

The fitting routine yields the resonance field, $B$, the half line width at half height, HWHM, and the resonance amplitude.

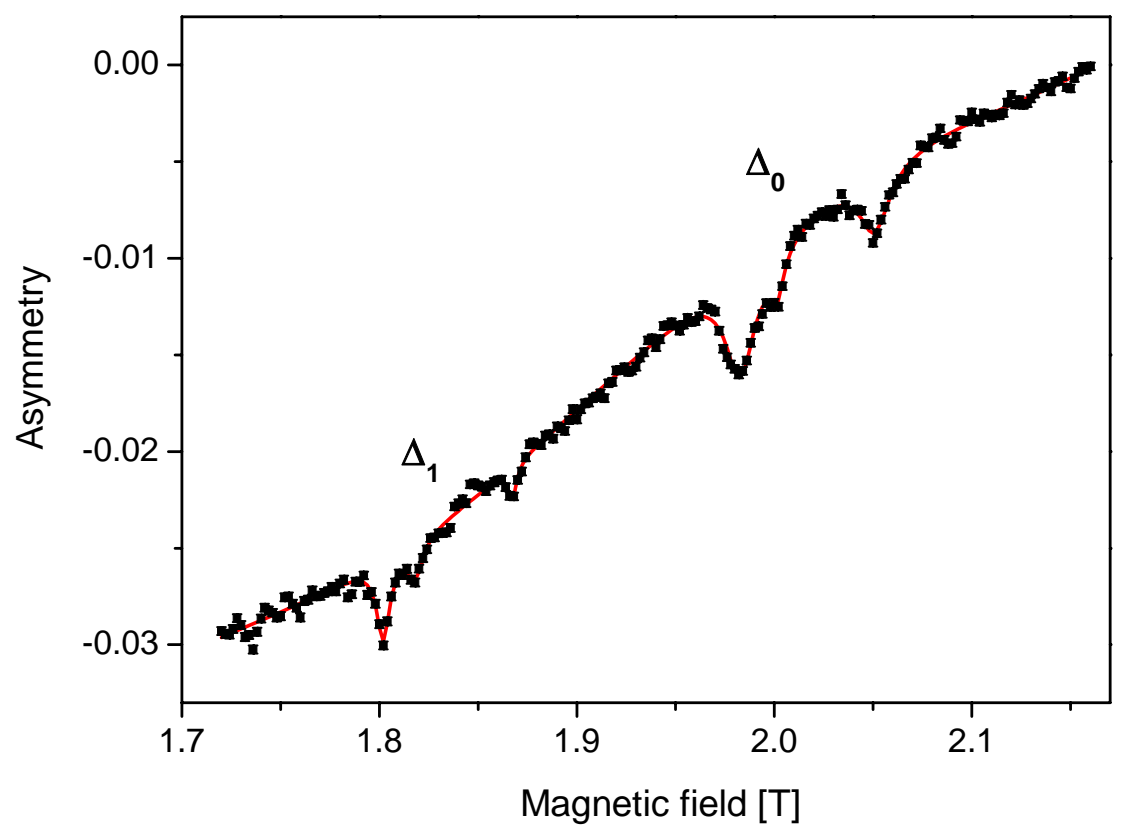

Figure 18: Fitted ALC- $\mu$ SR spectrum with subtracted background of $18 \mathrm{mM} 3-$ phenylpropan-1-ol in $15 \%$ aq. DHTAC emulsion at $75{ }^{\circ} \mathrm{C}$. The red line is attributed to the fitted six resonances of Lorentzian line shape.

However, an axial powder line shape using the same program was fitted to the experimental data in order to obtain the parallel uniaxial hyperfine coupling components, $D_{\|}^{\mu, e x p}$, see Subsection 4.1.5 and 4.2.5.

In order to determine the thermodynamic parameters of hydrophobic solvation of the series of phenyl alcohols (see Subsection 4.1.3) Origin 6.1 was applied.

In this case, the program gives as well as the resonance position, but instead of the HWHM the full line width at half height, FWHM is a fitting parameter. Moreover, in contrast to the previous procedure not the line intensity, but the area of the resonance is determined. 


\section{Chapter 4}

\section{RESULTS AND DISCUSSION}

In this Chapter the experimental results and their interpretation are presented. The experimental data corresponding to the partitioning of a series of phenyl alcohols in the binary system of DHTAC surfactant and in ternary and quaternary mixtures of SDS surfactant are discussed in Section 4.1 and in Section 4.2 respectively.

\subsection{The Binary System of DHTAC}

This system forms a simple (i.e., non-interdigitated) lamellar phase structure composed of lipid bilayers consisting of cationic dichain surfactant molecules DHTAC separated by water as determined by SAXS [84]. The surfactant amphiphiles aggregate to bilayers of ca. $5.2 \mathrm{~nm}$ thickness (head groups plus hydrophobic chain) that can be regarded as model cell membranes. They self-assemble into regularly spaced lamellar phase structures of ca. $30.2 \pm$ $0.4 \mathrm{~nm}$ periodicity in the $\mathrm{L}_{\beta}$ state [84]. This system was chosen because the solution state of the surfactant (and therefore bilayer properties) can be manipulated simply by changing the temperature. In the $\mathrm{L}_{\beta}$ phase in the absence of intercalated guest molecules the hydrocarbon chains of the double layers form a pseudo-crystalline structure where the alkyl chain motion is essentially frozen out. This phase melts on heating, leading to a liquid-like disordered hydrocarbon regime under retention of the overall lamellar structure that is termed $\mathrm{L}_{\alpha}$.

Using the ALC- $\mu$ SR technique we elucidate the partitioning and reorientational dynamics of five different cyclohexadienyl radicals derived from $\mathrm{C}_{6} \mathrm{H}_{5}\left(\mathrm{CH}_{2}\right)_{n} \mathrm{OH}$ with $2 \leq n \leq 5$, and $\mathrm{CH}_{3}\left(\mathrm{CH}_{2}\right)_{2} \mathrm{C}_{6} \mathrm{H}_{4}\left(\mathrm{CH}_{2}\right)_{2} \mathrm{OH}$ between the aqueous and the DHTAC surfactant environment in the temperature range $25-75^{\circ} \mathrm{C}$. The aromatic moiety where the muon spin probe will be attached is expected to penetrate deeper into the bilayer with increasing side chain length. In addition, to check for this effect two alcohols with the same total chain length and thus 
nominal hydrophobicity, one with $n=5$ and the other with a split chain $(n=2+3)$ in which the aromatic moiety is shifted back up nearer to the $\mathrm{OH}$ group, are measured.

An interesting question is how and whether at all such factors as:

a the temperature of the entire system;

口 the phenyl alcohol chain length;

口 the phenyl alcohol concentration;

a and the structure as well as the chemical nature of the probe molecules

can affect the probe molecule distribution in the DHTAC emulsion. Secondly, what is the dynamics of the muoniated radical in the DHTAC double layer, and is it possible to obtain any information from $\mathrm{ALC}-\mu \mathrm{SR}$ about the water penetration into the surfactant membrane. Finally, what is the concentration limit of slightly soluble phenyl alcohols for the ALC experiments in order to obtain good quality data.

\subsubsection{Solubility, Density and Calorimetric Measurements}

Figure 19 demonstrates that the solubility limit of phenyl alcohols in water at $25^{\circ} \mathrm{C}$ at $\lambda=$ $267 \mathrm{~nm}$ amounts to $18 \mathrm{mM}$ for $n=3,16 \mathrm{mM}$ for $n=4$ and $6 \mathrm{mM}$ for $n=5$. In the case of $n=$ $2+3$ molecule the solubility limit amounts to $5 \mathrm{mM}$ at $\lambda=271 \mathrm{~nm}$. As expected, the solubility decreases with increasing solute size, and thus with the hydrophobicity of the molecule.

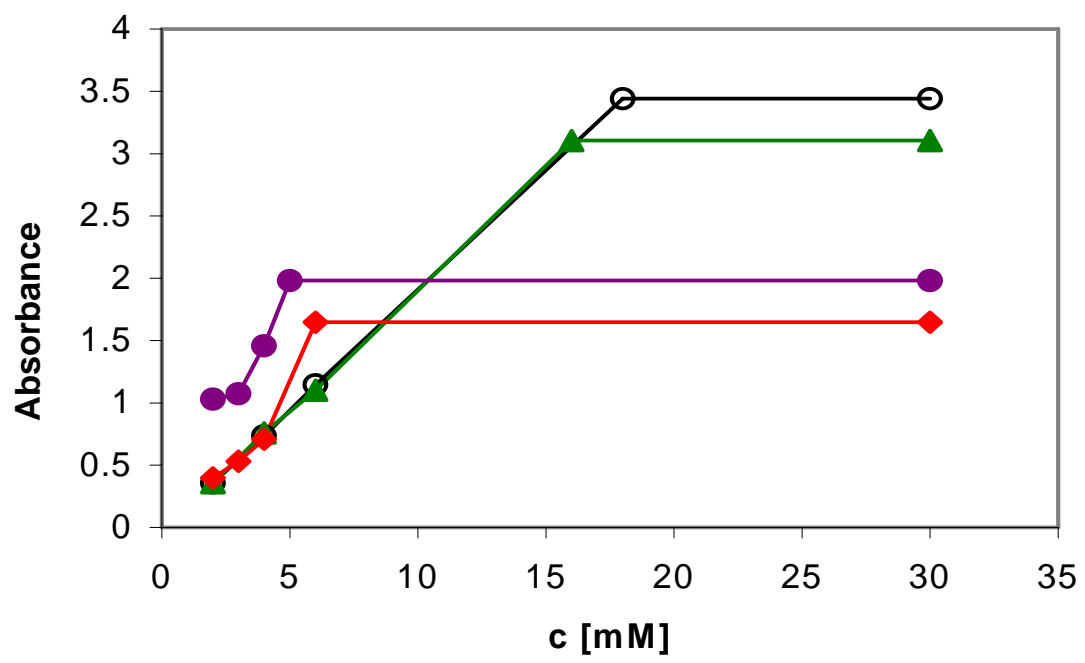

Figure 19: Solubility of phenyl alcohols in water at $25{ }^{\circ} \mathrm{C}: n=3(\bigcirc), n=4(\Delta), n=5(\diamond)$ and $n=2+3(\mathbf{O})$. 
The measured density of $p$-propyl-2-phenylethan-1-ol $(n=2+3)$ at $20{ }^{\circ} \mathrm{C}$ amounts to $0.96 \mathrm{~g} \mathrm{~cm}^{-3}$.

Calorimetric measurements were carried out to measure the temperature of the $\mathrm{L}_{\beta} / \mathrm{L}_{\alpha}$ phase transition that occurs in DHTAC dispersions in the absence and in the presence of added phenyl alcohols at concentrations of 5-40 mM. The DSC spectra reveal that the surfactant dispersions undergo only a single phase transition. Depending on the hydrocarbon chain length and concentration of the added alcohol the temperature of this phase transition increases from 61 to $64{ }^{\circ} \mathrm{C}$ (see Figure 20), which is consistent with findings in literature [97].

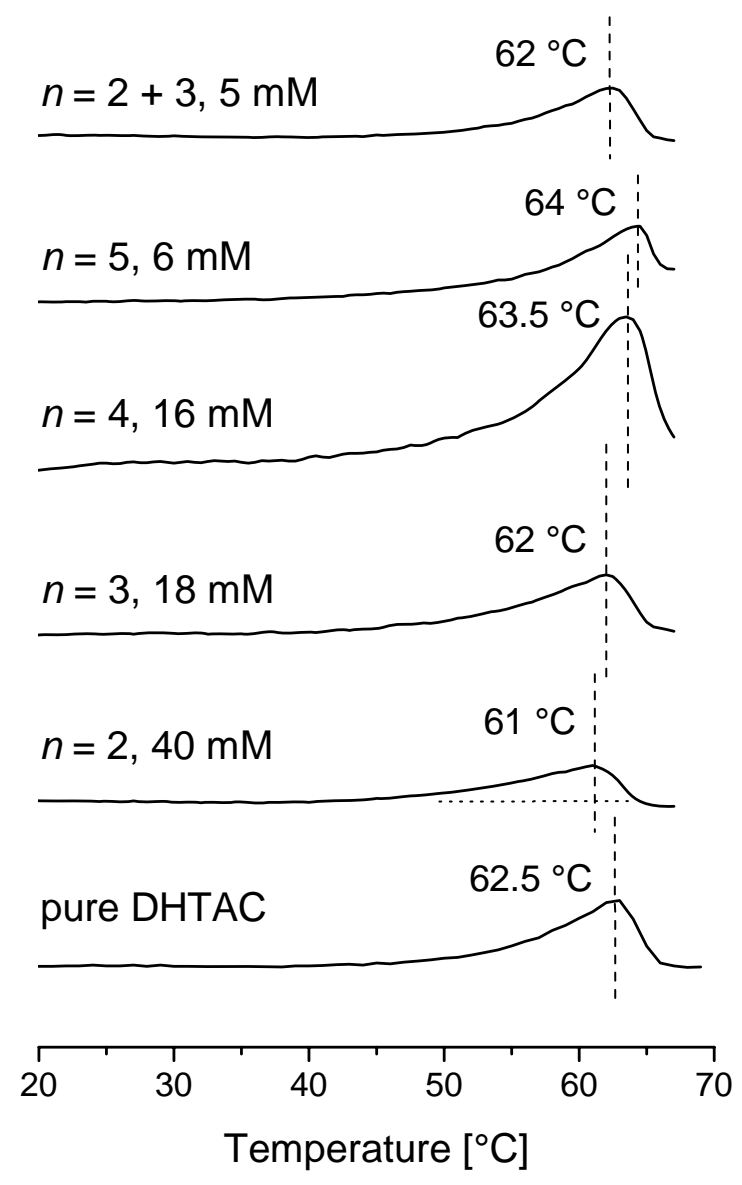

Figure 20: DSC traces showing the $\mathrm{L}_{\beta} / \mathrm{L}_{\alpha}$ phase transition of $15 \%$ aq. DHTAC without and with added phenyl alcohol at 5-40 mM concentration as a function of $n\left(\mathrm{CH}_{2}\right)$. The dashed vertical line marks the transition temperature. The dotted baseline drawn for $n=2$ demonstrates that the phase transition is broad and starts well below its nominal temperature, leading to $\mathrm{L}_{\beta} / \mathrm{L}_{\alpha}$ phase coexistence over a temperature range of nearly $20^{\circ} \mathrm{C}$. 


\subsubsection{Partitioning of Phenyl Alcohols}

\subsubsection{Probe Molecule Location}

\section{Determination of the \% Aqueous Character}

In $\mathrm{ALC}-\mu \mathrm{SR}$ experiments the resonance fields can be accurately measured and used to monitor the local environments of the spin probe radicals, owing to the dependence of the hyperfine coupling constants on the polarity of the environment. Such a dependence results in shifts of the resonance fields, as plotted in Figure 21 for the three isomers of the radicals derived from the $n=3$ alcohol.

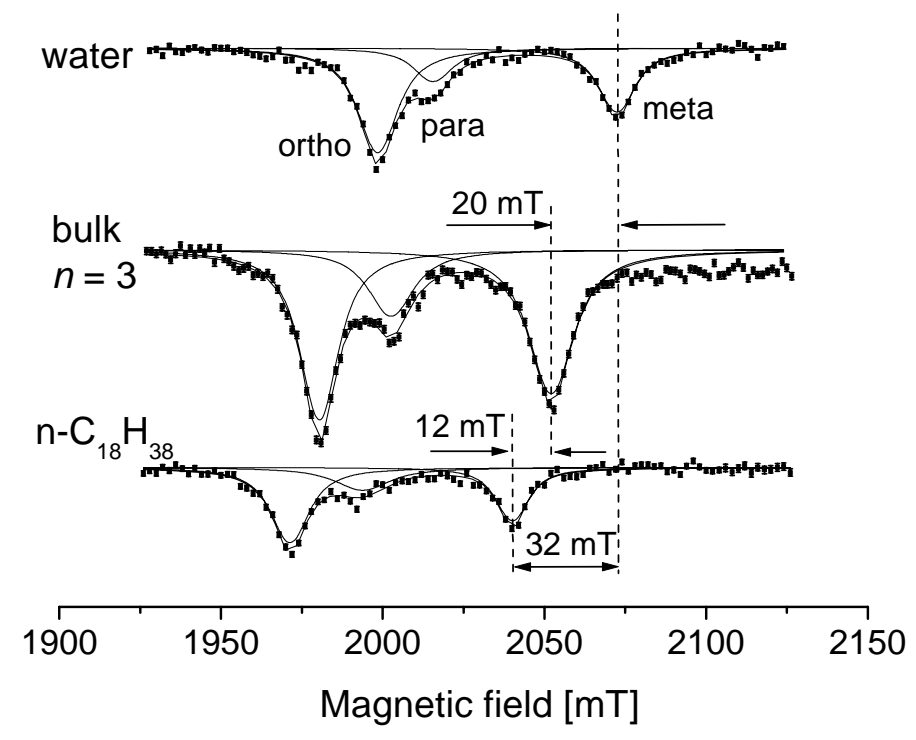

Figure 21: Methylene $\Delta_{0}$ resonances of the three isomers of muoniated 3-phenylpropan-1-ol in water (top), in bulk liquid alcohol (middle), and in octadecane (bottom) at $75{ }^{\circ} \mathrm{C}$. The assignment of the lines to specific isomers is analogous to that of the radicals derived from 2phenylethan-1-ol [84,98].

It is seen that the resonances shift down-field by $>30 \mathrm{mT}$ when the environment changes from water, through phenylpropanol, to a hydrocarbon, here octadecane.

In the case of the $n=2+3$ alcohol the para position is blocked by the propyl group, therefore only the two $\Delta_{0}$ resonances of the remaining isomers (denoted by $x$ and $y$ since the assignment is uncertain) are observed in octadecane. In aqueous environment, however, the two isomers are not distinguishable so that only a single signal appears, which shows that the 
hyperfine couplings of the two isomers respond differently to the environment so that they are the same in aqueous but not in a hydrocarbon environment (Figure 22).

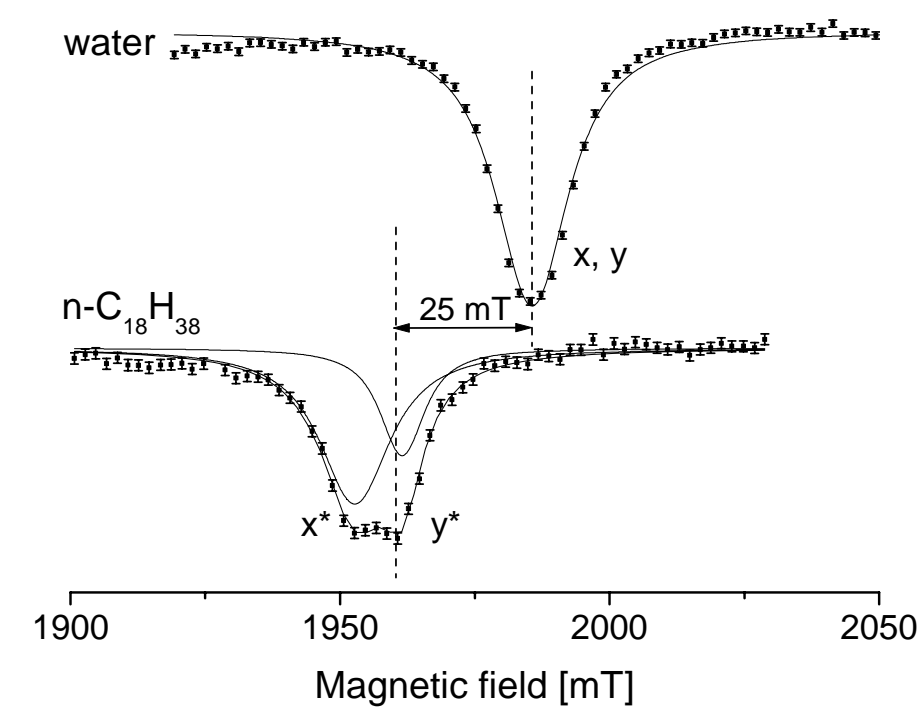

Figure 22: Methylene $\Delta_{0}$ resonances of the two isomers of muoniated para-propyl-2phenylethan-1-ol in water (top), and in octadecane (bottom) at $75^{\circ} \mathrm{C}$.

Since resonance fields can be measured with an accuracy of ca. $0.5 \mathrm{mT}$ this permits an accurate determination of the local solvent polarity, given in terms of an 'aqueous character' which is defined as a fractional shift of the resonance between the resonance fields in water (100\% aqueous character) and octadecane (0\% aqueous character) $[83,84]$,

$$
\text { \%aq.char. }=\frac{B_{\text {DHTAC }}-B_{\text {Octadecane }}}{B_{\mathrm{H}_{2} \mathrm{O}}-B_{\text {Octadecane }}}
$$

where $B_{\text {DHTAC }}, B_{\text {Octadecane }}$ and $B_{\mathrm{H}_{2} \mathrm{O}}$ are the resonance positions in the DTHAC dispersions, and in the reference states octadecane and water, respectively.

To explain the principles of the analysis, at first the case of 3-phenylpropan-1-ol will be discussed in some detail. Thereafter, the differences observed for the higher alcohols relative to $n=3$ will be pointed out. The case of 2-phenylethan-1-ol $(n=2)$ was reported in some detail previously $[6,84,98]$. As discussed in Subsection 2.3 there are five main criteria to retrieve the information of interest: (i) the position of the $\Delta_{0}$ resonances provides the polarity of the environment and thus the location of the guest molecule, (ii) the presence of a $\Delta_{1}$ resonance is clear evidence that the guest species is in an anisotropic environment, i.e. incorporated in, or associated to, the bilayer, (iii) if more than one $\Delta_{0}$ resonance is present for 
each isomer this means that the guest species resides in two different environments between which there is no fast exchange, (iv) from the areas of the $\Delta_{0}$ resonances the thermodynamic parameters for the transfer of the guest species from the water to the surfactant bilayer can be obtained, and (v) the width and the intensity of a $\Delta_{1}$ resonance relative to its $\Delta_{0}$ counterpart provides information about the extent of dynamic averaging of the hyperfine anisotropy and thus qualitatively about the order parameter of the guest species in the bilayer.

Typical ALC spectra of $n=3$ in $15 \%$ aq. DHTAC solutions recorded as a function of temperature are presented in Figure 23.

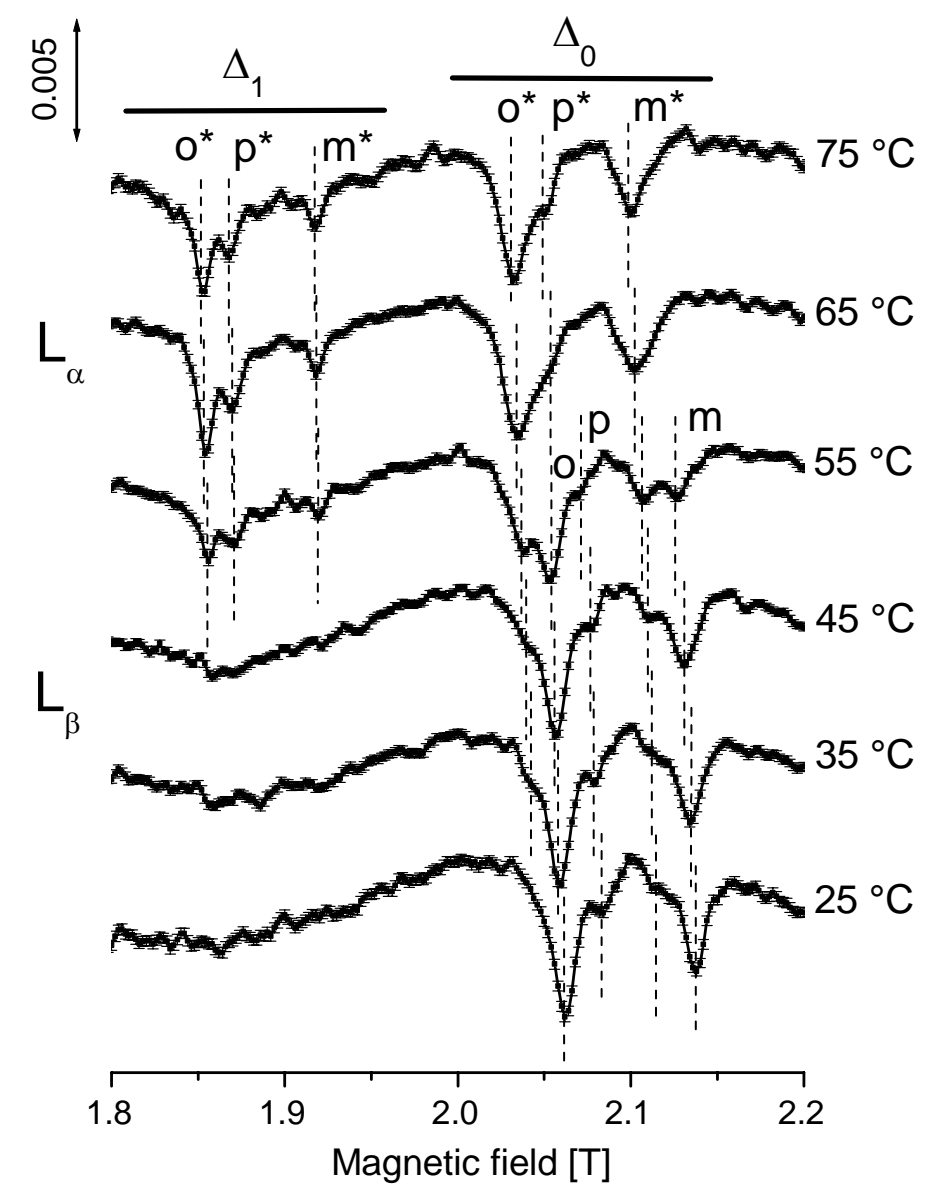

Figure 23: ALC- $\mu$ SR spectra of $18 \mathrm{mM}$ 3-phenylpropan-1-ol in 15\% aq. DHTAC emulsion. The asterisk marks the peaks relating to the radical incorporated within the bilayer. The other peaks correspond to the aqueous phase. Background correction was done by baseline subtraction.

Three states of the phenylpropanol distribution can be distinguished. First, in the high temperature $\mathrm{L}_{\alpha}$ phase, single sets of three $\Delta_{1}$ and $\Delta_{0}$ resonances of the ortho, meta and para 
isomers are detected. The positions of the $\Delta_{0}$ lines are found at field values which are close to the resonance positions in octadecane, indicating that the phenyl alcohol molecules reside mostly within the surfactant bilayer. This assignment is supported by the presence of the $\Delta_{1}$ resonances.

Secondly, just below the phase transition, at $55{ }^{\circ} \mathrm{C}$, the $\Delta_{1}$ lines are still present, although they are much weaker than in the $\mathrm{L}_{\alpha}$ phase. This clearly shows that a significant fraction of the muoniated radicals are present in the DHTAC membrane. Furthermore, the appearance of five $\Delta_{0}$ peaks, with probably a sixth line overlapping, is evidence for the coexistence of the alcohol in the hydrocarbon and the water regions.

This coexistence reveals that the $\mathrm{L}_{\beta}$ phase is still sufficiently soft just below the phase transition temperature to allow the phenyl alcohol to penetrate the membrane. The polarity diagram that quantifies the information of the entire phenyl alcohol series will be discussed further below (Subsection 4.1.2.3).

Finally, in the low temperature $\mathrm{L}_{\beta}$ phase, the spectra between $25{ }^{\circ} \mathrm{C}$ and $45{ }^{\circ} \mathrm{C}$ show no sign of $\Delta_{1}$ resonances, and a large shift of the $\Delta_{0}$ lines to higher fields that are typical for the aqueous phase. It is also important to notice that with decreasing temperature, the area of the $\Delta_{0}$ peaks, corresponding to the molecule located in the DHTAC membrane $\left(o^{*}, m^{*}, p^{*}\right)$, becomes smaller, in comparison with the area related to the aqueous part $(o, m, p)$. It means that almost the entire fraction of the radicals is expelled from the nonpolar medium into the aqueous regime, and only a trace of 3-phenylpropan-1-ol is left in the bilayer, which is no longer detectable, by $\Delta_{1}$ resonances.

Figure 24 displays the spectra obtained for $n=4$ radicals. They reveal a similar trend as just discussed for 3-phenylpropan-1-ol, although the $\Delta_{0}$ resonances of the aqueous fraction in the $L_{\beta}$ phase seem to be slightly smaller here, which is attributed to the increased hydrophobicity of this species, resulting in a higher affinity to the anisotropic environment.

Furthermore, in contrast to $n=3$, the spectrum obtained with 4-phenylbutan-1-ol reveals very weak $\Delta_{1}$ lines down to $45{ }^{\circ} \mathrm{C}$, indicating the presence of $\mathrm{L}_{\beta} / \mathrm{L}_{\alpha}$ phase coexistence or, more generally, a residual alcohol concentration within the DHTAC membrane. 


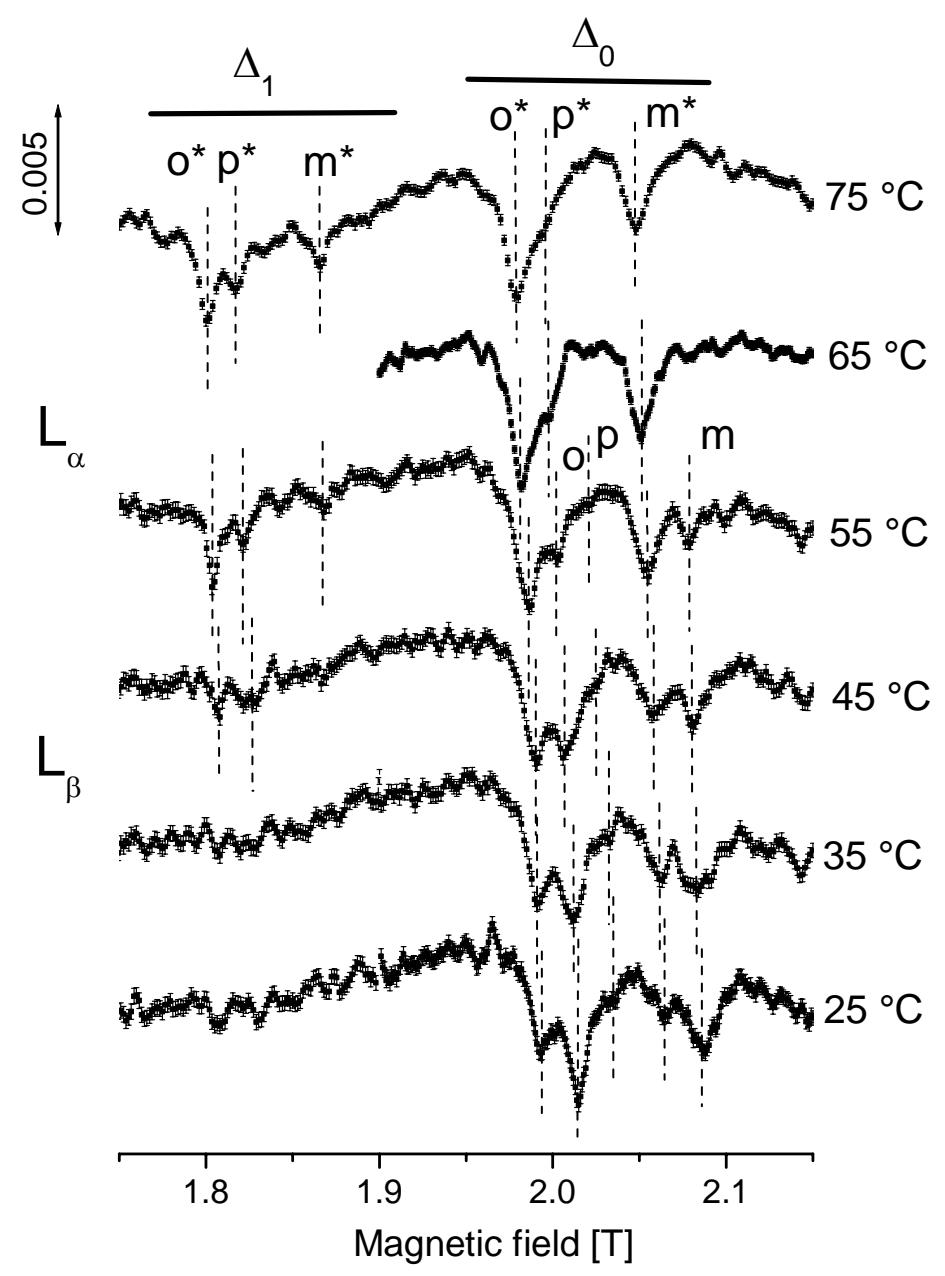

Figure 24: ALC- $\mu \mathrm{SR}$ spectra of $16 \mathrm{mM}$ 4-phenyl-butan-1-ol in 15\% aq. DHTAC. The asterisk marks the alcohol located within the bilayer. Other peaks relate to water. Background correction was done by baseline subtraction.

In the case of $n=2+3$ only two states of probe molecule partitioning can be defined. In the temperature range $75-55^{\circ} \mathrm{C}$, the two isomers $\left(x^{*}, y^{*}\right)$ of the alcohol are located within the surfactant bilayer, which is confirmed by the presence of two $\Delta_{1}$ lines (see Figure 25).

Below $55{ }^{\circ} \mathrm{C}$, the two $\Delta_{1}$ resonances are still observed, although at lower intensity than in the $\mathrm{L}_{\alpha}$ phase. Simultaneously, the $\Delta_{0}$ feature broadens significantly towards the high field side, suggesting that the two lines $\left(x^{*}, y^{*}\right)$ are overlapped by a third one that is common for the two species $(x, y)$ in the aqueous environment of the equality of the corresponding hyperfine couplings (compare Figure 22). These facts demonstrate that also $n=2+3$ coexists within the aqueous and nonaqueous environments between 25 and $45^{\circ} \mathrm{C}$. 


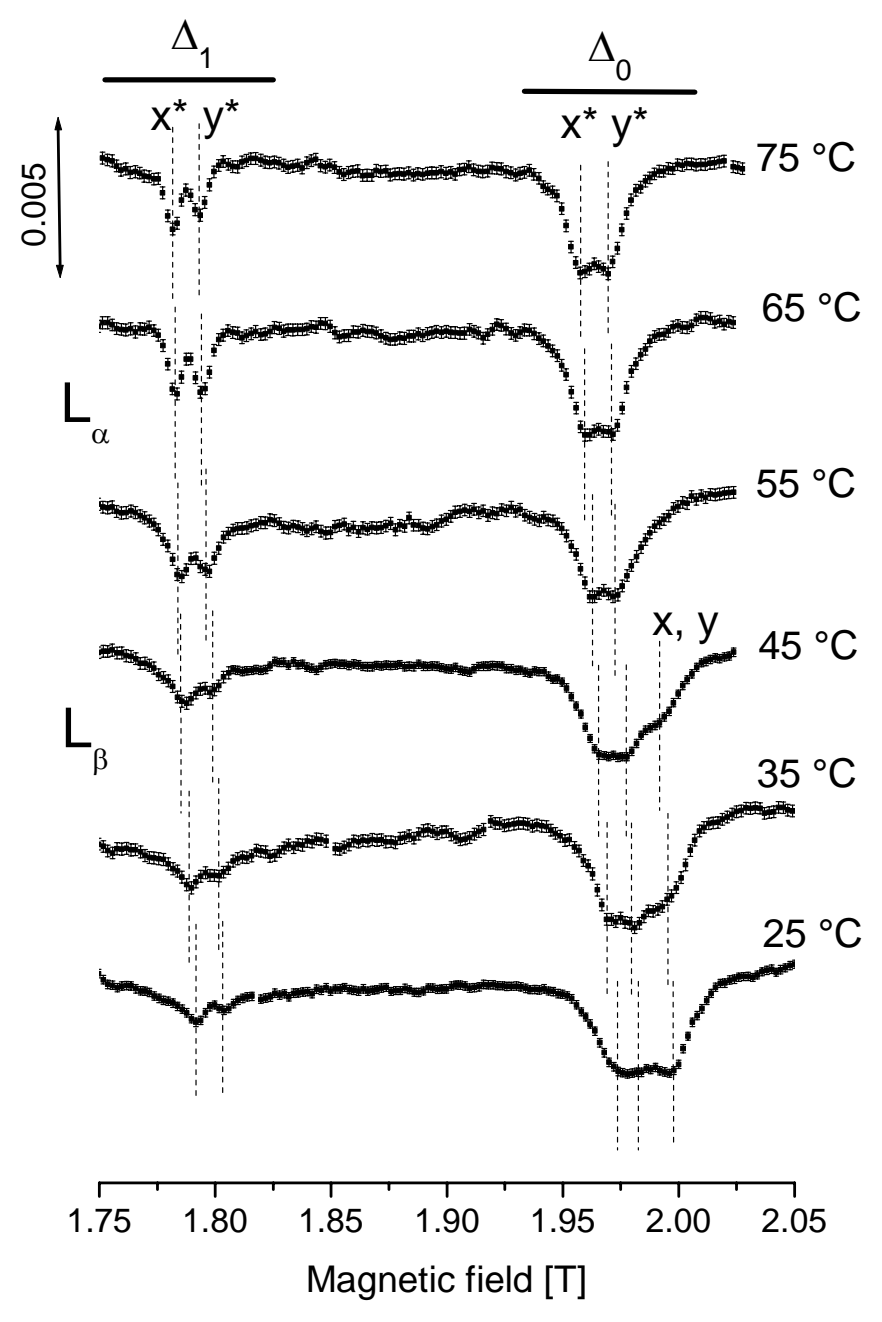

Figure 25: ALC- $\mu$ SR spectra of $4 \mathrm{mM}$ para-propyl-2-phenylethan-1-ol in $15 \%$ aq. DHTAC. The asterisk marks the alcohol located within the bilayer. Other peaks relate to water. Background correction was done by baseline subtraction.

Figure 26 displays the spectra obtained with 5-phenylpentan-1-ol $(n=5)$. In the field range near $1.8 \mathrm{~T}$ the behaviour is analogous to that of $n=2+3$, with clear $\Delta_{1}$ features in the entire temperature range but with lower amplitudes in the $\mathrm{L}_{\beta}$ phase below $50{ }^{\circ} \mathrm{C}$.

The $\Delta_{0}$ range shows coexistence of the spin probe between the bilayer and the aqueous environments, with temperature dependent fractions as revealed most clearly by the changing amplitudes of the $\mathrm{m}$ and $\mathrm{m}^{*}$ isomers. Obviously, in the $\mathrm{L}_{\beta}$ phase the increased alkyl chain length leads to a similar affinity of this alcohol for the two environments. 


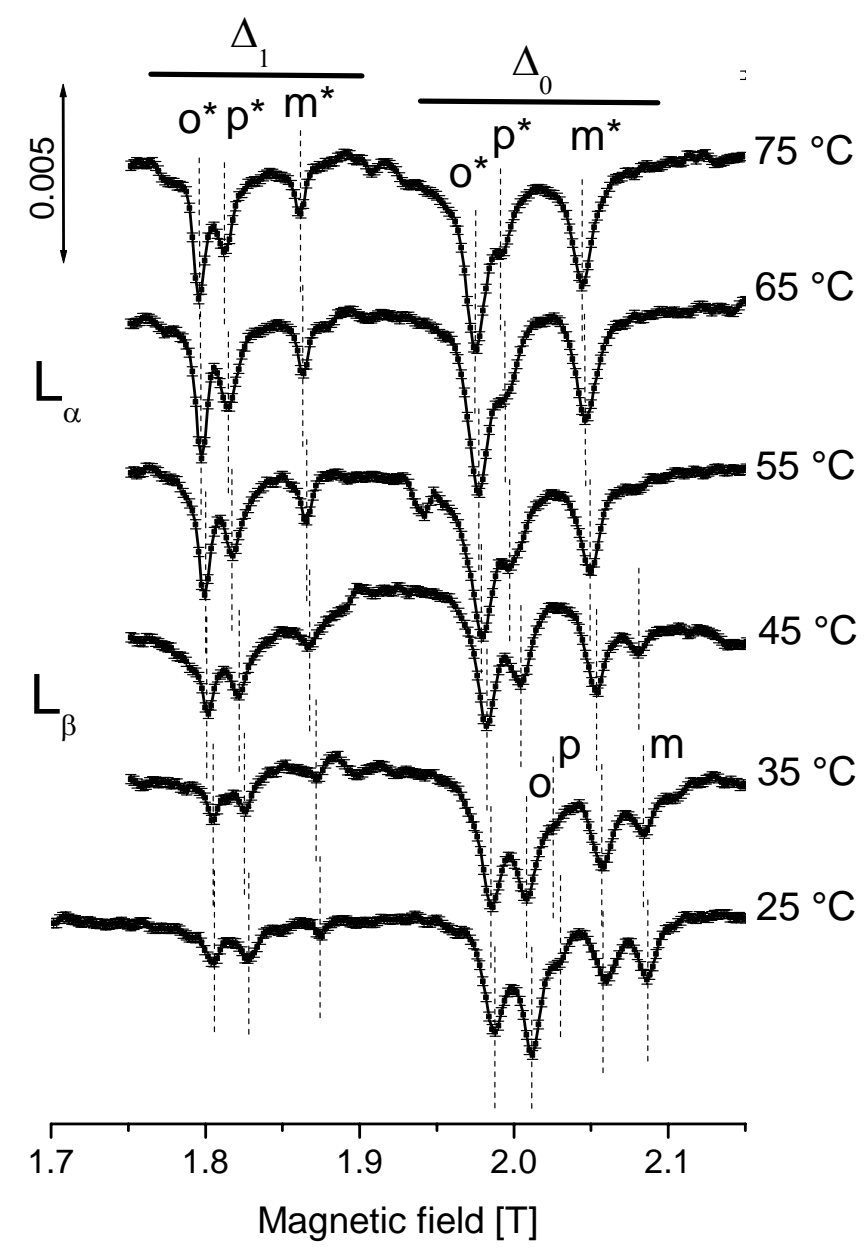

Figure 26: ALC- $\mu$ SR spectra of $40 \mathrm{mM}$ muoniated 5-phenylpentan-1-ol in 15\% aq. DHTAC dispersion. Background correction was done by baseline subtraction. The scale bar is for the asymmetry.

\subsubsection{Concentration Dependence}

Furthermore, the concentration dependence of the probe molecule partitioning was tested. The results for a $40 \mathrm{mM}$ and a $6 \mathrm{mM} n=5$ phenyl alcohol are shown in Figures 26 and 27.

It is evident that the fraction of 5-phenylpentan-1-ol in the apolar medium increases with concentration (see Figure 26, 27). It is confirmed by significantly larger $\Delta_{1}$ resonances and the presence of only three $\Delta_{0}$ lines at $55^{\circ} \mathrm{C}$ in the case of $40 \mathrm{mM}$ of $n=5$ species. It indicates that the radical already penetrates the interface to $100 \%$ at this temperature (see Figure 26), whereas for a $6 \mathrm{mM}$ solution the alcohol is fully incorporated in the membrane just above the phase transition (see Figure 27). 


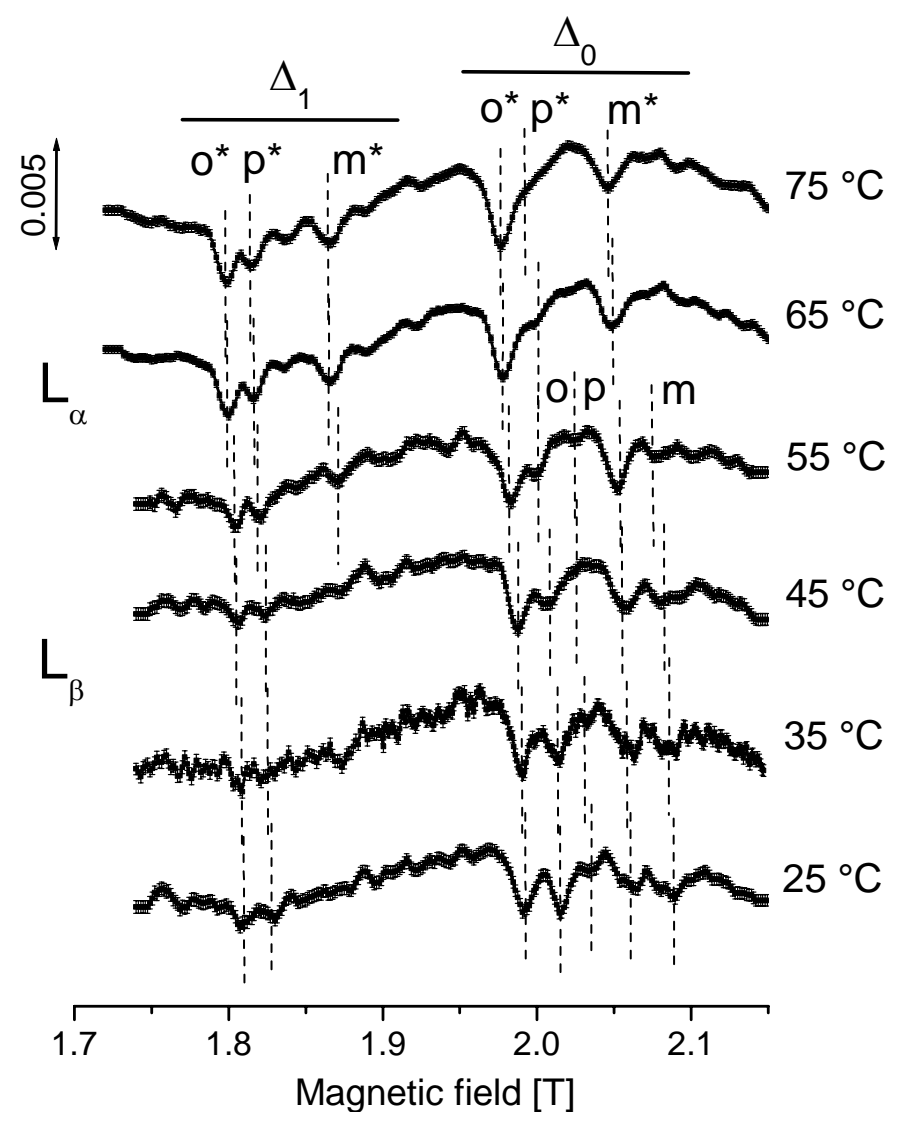

Figure 27: ALC- $\mu$ SR spectra of $6 \mathrm{mM}$ muoniated 5-phenylpentan-1-ol in 15\% aq. DHTAC dispersions. Background correction was done by baseline subtraction.

\subsubsection{Environment Polarity}

\section{Determination of Cosurfactant Partitioning}

The affinity of the five phenyl alcohols for the aqueous versus nonaqueous environment is illustrated for each of the ortho, para and meta isomers in the polarity diagrams in Figure 28. In the high temperature $\mathrm{L}_{\alpha}$ phase, all three isomers of all cosurfactants sense an aqueous character of $<60 \%$, and they clearly show a systematic behaviour. With increasing alcohol chain length the aqueous character of the ortho and meta isomers decreases from approximately $55 \%$ aq. for the $n=2$ radical [84] to roughly $20 \%$ aq. for the $n=5$ radical [83]. The somewhat different behaviour of the para isomer will be discussed later. In none of the cases does the phenyl ring sense an environment which belongs to the pure hydrocarbon environment in the centre of the bilayer ( $0 \%$ aq.). 

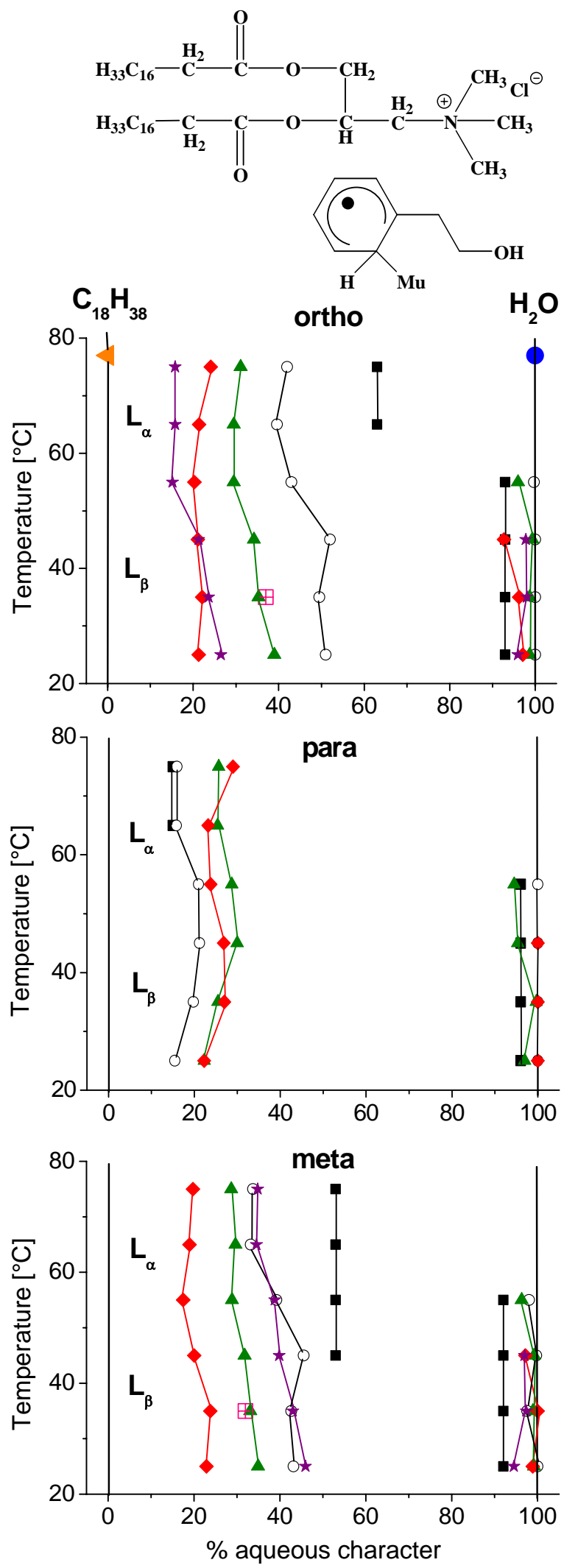

Figure 28: Structure of the dichain cationic surfactant DHTAC and of the ortho-2phenylethan-1-ol with corresponding polarity diagram for: $n=2(\mathbf{\square}), n=3(\bigcirc), n=4(\mathbf{\Delta}), n$ $=5(\diamond)$ and $n=2+3(\star)$ in $15 \%$ DHTAC dispersion, in water $(\bigcirc)$, in ethyl acetate $(\boxplus)$ and in octadecane $(\varangle)$, respectively. 
Below the phase transition to the $\mathrm{L}_{\beta}$ phase, a doubling of the $\Delta_{0}$ resonances occurs (Subsection 4.1.2.1) and this is the reason why two lines are present for each isomer in the polarity diagrams. It indicates that the coexistence within the bilayer and the water regime is a common phenomenon for almost all phenyl alcohols. 2-phenylethan-1-ol is an exception in this case because only a single set of $\Delta_{0}$ lines appears [84] between 25 and $35^{\circ} \mathrm{C}$, indicating that the alcohol does not coexists within the bilayer and water region. 2-phenylethan-1-ol is also not totally incorporated in the aqueous regime, because it shows only $93 \%$ aq. character. The explanation of this phenomenon is given in Section 4.1.4.

It is worth noticing that the difference in aqueous character between the $\mathrm{L}_{\alpha}$ and $\mathrm{L}_{\beta}$ phases in the surfactant region amounts to roughly 5-10\% (see Figure 28), meaning that the radicals do not penetrate into the crystalline hydrocarbon chain structure of the $\mathrm{L}_{\beta}$ phase as deeply as into the liquid crystalline structure in their $\mathrm{L}_{\alpha}$ phase, while they remain anchored at the surfactant/water interface. A $0.17 \mathrm{M}$ aqueous solution of $\mathrm{N}\left(\mathrm{CH}_{3}\right)_{4} \mathrm{Cl}$ was also measured to simulate the effect of the trimethylammonium head group of DHTAC [84]. The aqueous character observed with the $n=2$ spin probe in these solutions is close to that in pure water, indicating that a location of the spin probe near this head group would be difficult to distinguish from the aqueous environment.

In principle there are two explanations for the different behaviour in the two phases: (i) The molecules are in fast dynamic equilibrium between the aqueous and the hydrocarbon region, with an increasing affinity to the hydrocarbon environment with increasing chain length. (ii) The guest molecules are located entirely within the surfactant layer, but there is a polarity gradient, and the phenyl ring senses a more hydrocarbon-like environment the longer its side chain with the $\mathrm{OH}$ group is. This would be the case if the alcohol is anchored near the interface to the aqueous environment, as it is suggested on top of Figure 28.

It is seen in Figure 28 that for all cases except for $n=2$ (which will be discussed separately in Section 4.1.4) the high temperature behaviour extends smoothly down to the $\mathrm{L}_{\beta}$ phase at lower temperature. In this phase we know that equilibration is slow on a timescale of $10^{-5} \mathrm{~s}$ since we observe the alcohol separately both in the aqueous and the surfactant environment, and the polarity sensed in the surfactant is nearly the same as in the $\mathrm{L}_{\alpha}$ phase. The polarity in the aqueous regime is close to $100 \%$ aq. as expected, again with the (marginal) exception for $n=2$. 
By default at first we are left to accept the second explanation. For the ortho and meta isomers in the bilayer Figures 29a, b clearly show a decreasing polarity with increasing hydroxyalkyl chain length with $2 \leq n \leq 5$, both at 35 and $65{ }^{\circ} \mathrm{C}$.
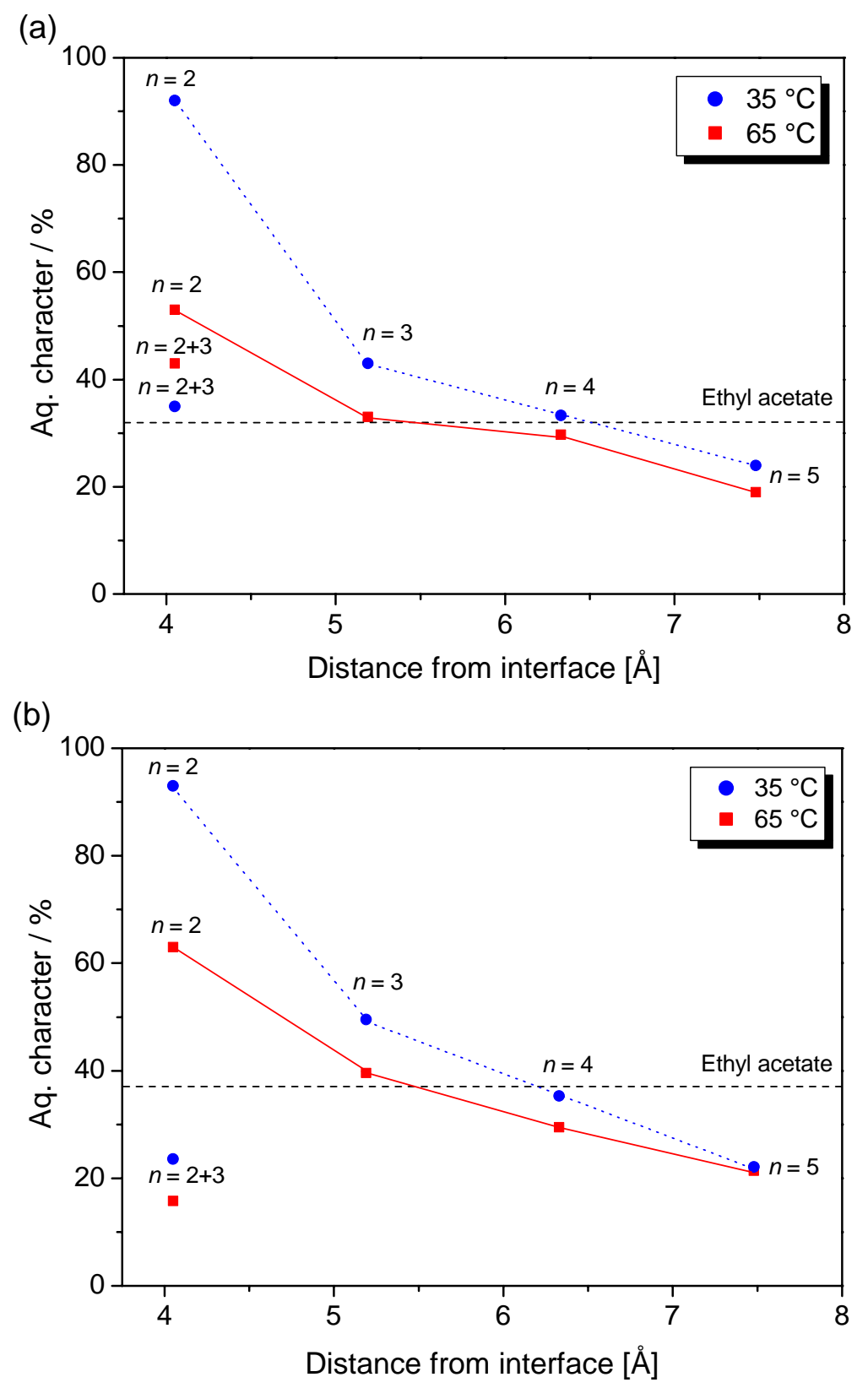

Figure 29: Water gradient into the lamellar phase as determined from the meta (a) and the ortho (b) isomer of the series of phenyl alcohols.

This suggests the presence of a gradient of water molecules in the DHTAC bilayer. It should be noted in this context that the aromatic part of the alcohol shows a strong preference for hydrogen bonding with two water molecules, one to each face of the phenyl ring [99]. We 
recall that it is known from conventional spin label experiments that there is indeed a gradient of water molecules which extends to some depth into a biomembrane containing 5 or 40 mol\% cholesterol [100-102]. A trough-like distribution of water molecules across the membrane with a steep drop near carbon number 8 of the lipid chain was found [102].

Recently, it was also shown that water diffusion into the ethoxy part of the surfactant chain is possible [103]. Another approach that provides evidence for water penetration into the lipid head group and interchain moiety of the bilayers is time-resolved fluorescence spectroscopy [104]. This study shows that the amount of water residing within the lipid chain of a surfactant membrane increases upon the addition of short-chain n-alcohols (methanol to propanol) and decreases for longer-chain n-alcohols (butanol to octanol). By contrast, the level of hydration within the head group region increases with n-alcohol chain length. The above phenomenon is believed to be due to the increased head group spacing and the bilayer chain packing free volume by the addition of short-chain n-alcohols, allowing more water molecule penetration into the membrane, whereas the increased presence of methylene groups reduces the void volume in the interchain region.

Two further points should be noted in this context: First of all, the chains of our phenyl alcohol probe molecules are short, and if they are anchored with the $\mathrm{OH}$ group at the interface the aromatic ring is unlikely to fully reach the hydrocarbon environment. The surfactant ester group itself is expected to account for a polarity of $32-37 \%$, as revealed by measurements in water-free bulk liquid ethyl acetate [84]. We measure values from $50 \%$ down to $20 \%$, depending on the phenyl alcohol chain length. Therefore the aromatic rings seem to be located near the ester group, or somewhat further inside the bilayer, and the head groups compete with the guest molecule for these water molecules.

The para isomers of the $2 \leq n \leq 4$ alcohols seem to be less selective and show an aqueous character of between 15 and $30 \%$ only. We recall that this isomer showed significant deviations from the linear polarity behaviour already in isotropic solution [105,106].

The para-propyl-phenylethanol $(n=2+3)$ alcohol deviates from the behaviour considered above and needs to be discussed separately. It shows roughly $40 \%$ aqueous character for one $\left(y^{*}\right)$ and $20 \%$ for the other isomer $\left(x^{*}\right)$ in both phases, which is less than the values found for 2-phenylethan-1-ol $(n=2)$, the other probe with the same alkanol chain length (see Figure 28). This may simply reflect the increased hydrophobicity due to the propyl group. However, the numbers for the two isomers differ by a factor of two, which may indicate a different 
influence on the chain packing and water penetration but should be viewed with caution because of the large error which is due to the unresolved lines.

Our results and their interpretation are further supported by the solubility measurements of 2-phenylethan-1-ol $(n=2)$ in didodecyldimethylammonium bromide (DDAB) surfactant vesicles and dodecyltrimethylammonium bromide (DTAB) micelles as reported by Kondo and coworkers [107]. These authors found that 2-phenylethan-1-ol is solubilised in the hydrophilic region of vesicle bilayers and micelles, with the hydroxyl head group of the alcohol attracted to the charged head group of DDAB and DTAB surfactants. However, the lower packing in the head group moiety of vesicles compared with micelles leads to much stronger bonding of 2-phenylethan-1-ol in the hydrophilic region of DDAB cationic surfactant. Furthermore, they concluded that solutes such as aliphatic alcohols and phenols have their head groups anchored in the polar/ionic outer region of typical ionic amphiphiles, while the aliphatic or aromatic moieties of these solutes tend to be solubilised directly within the nonpolar core (the ester group is absent in these surfactants).

Furthermore, as it was mentioned before, the calorimetric measurements allowed determining the temperature of the phase transition that amounts to $61-64{ }^{\circ} \mathrm{C}$ depending on the type of alcohol incorporated in the DHTAC mixture. The phase transition extends over a range of about $10{ }^{\circ} \mathrm{C}$, reflecting the coexistence of the $\mathrm{L}_{\alpha}$ and $\mathrm{L}_{\beta}$ phases, or perhaps the distribution of chain lengths within the surfactant.

\subsubsection{Partitioning Equilibrium at the Water/Surfactant Interface}

The explanation of the mechanisms that regulate the guest molecule distribution in surfactant emulsions requires an adequate understanding of thermodynamic parameters at a molecular level. It is well known that apolar solutes such as higher phenyl alcohols are only slightly miscible with water, which is attributed to their hydrophobicity. At a molecular level, the transfer of the muoniated alcohol from the aqueous environment into the surfactant bilayer, which is characteristic for the nonclassical hydrophobic effect $[39,46]$ and can be described by the equilibrium,

$$
\operatorname{radical}(\text { water }) \rightleftarrows \operatorname{radical(bilayer)} \text {. }
$$


Introduction of the solute into water, however, involves the disruption of its interactions with the DHTAC amphiphiles, a cavity creation in water, and the initiation of alcohol-water interactions which is typical for the classical hydrophobic effect [36].

The two environments are observed as separate resonances as long as the exchange equilibrium is slow. The critical timescale is given by the difference of the oscillation frequencies of the spin system at the avoided level crossing. For $\Delta_{0}$ resonances of cyclohexadienyl type radicals this frequency is typically on the order of $0.6 \mathrm{MHz}$ [81] and the difference between the two environments amounts to ca. $1 \%$ of this value, so that the critical timescale for separate observation is on the order of $10^{-5} \mathrm{~s}$ [109].

The work required to transfer one mol of solute molecules from the water into the bilayer is given by the change of the free energy $\Delta G_{\mathrm{tr}}$. In terms of thermodynamics, $\Delta G_{\mathrm{tr}}<0$ denotes higher solubility of the solute in the bilayer environment than in water.

The simultaneous observation of the $\Delta_{0}$ resonances for the aqueous and the bilayer moieties allows to estimate the free energy of transfer. From the well resolved split of the $\Delta_{0}$ lines $\Delta G_{\text {tr }}$ was calculated using the following relation:

$$
\Delta G_{\mathrm{tr}}=-R T \ln K_{\mathrm{eq}}=-R T \ln \left(\frac{x_{\text {bilayer }}}{x_{\text {water }}}\right)
$$

where the equilibrium coefficient $K_{\text {eq }}$ was evaluated from the ratio of areas $x_{\mathrm{i}}$ of the $\Delta_{0}$ lines belonging to the corresponding environments. The resulting $\Delta G_{\text {tr }}$ values listed in Table 6 are consistent with the picture of probe molecule location introduced in Subsection 4.1.2.1.

The values of the free energy change for the $n=2$ species are estimated by extrapolation from $n=3,4$ and 5 , by a linear fit to indicate the probable trend.

$\Delta G_{\text {tr }}$ decreases slightly with temperature, which moves the equilibrium to the right hand side. By eye this is seen best in Figures 23-26 for the meta $\Delta_{0}$ resonance in the $\mathrm{L}_{\beta}$ phase. The transfer of the radicals with $2 \leq n \leq 4$ from water to the DHTAC bilayer results in a positive free energy change between $25^{\circ} \mathrm{C}$ and $45^{\circ} \mathrm{C}$.

The molecule with $n=5$ reveals less positive $\Delta G_{\text {tr }}$ values than the smaller alcohols due to its hydrophobocity and thus has a higher affinity for the nonaqueous surfactant regime. Unexpectedly, $\Delta G_{\operatorname{tr}}$ remains negative for the $n=2+3$ radical in the $\mathrm{L}_{\beta}$ phase and is significantly smaller than for $n=5$ (although the two species have the same chain length), which indicates more extensive bilayer penetration (Table 6). 
Table 6: Gibbs free energy change $\left(\Delta G_{\mathrm{tr}}\right.$ in $\left.\mathrm{kJ} \mathrm{mol}^{-1}\right)$ for the transfer of the phenyl alcohols from the water into the bilayer at their $25{ }^{\circ} \mathrm{C}$ solubility limit as measured from the $\Delta_{0}$ resonances of the meta isomers $\left(m, m^{*}\right)$.

\begin{tabular}{|c|c|c|c|c|c|}
\hline \multicolumn{2}{|c|}{ Phenyl alcohol, concentration } & \multirow{2}{*}{$\frac{25^{\circ} \mathrm{C}}{\text { ca. } 4.9^{\mathrm{a}}}$} & \multirow{2}{*}{$\frac{35^{\circ} \mathrm{C}}{\text { ca. } 5.3^{\mathrm{a}}}$} & \multirow{2}{*}{$\frac{45^{\circ} \mathrm{C}}{2.1 \pm 0.6}$} & \multirow{2}{*}{$\frac{55^{\circ} \mathrm{C}}{0.1 \pm 0.7}$} \\
\hline (a) & $n=2,40 \mathrm{mM}$ & & & & \\
\hline & $n=3,18 \mathrm{mM}$ & $3.1 \pm 0.7$ & $3.7 \pm 0.9$ & $3.0 \pm 0.4$ & $-0.2 \pm 0.3$ \\
\hline & $n=4,16 \mathrm{mM}$ & $3.7 \pm 0.5$ & $2.6 \pm 0.4$ & $1.4 \pm 0.4$ & $-2.8 \pm 0.5$ \\
\hline & $n=5,6 \mathrm{mM}$ & $1.4 \pm 0.5$ & $1.0 \pm 0.5$ & $1.0 \pm 0.6$ & $-0.8 \pm 0.6$ \\
\hline \multicolumn{2}{|c|}{$n=2+3, y^{*}$ isomer, $5 \mathrm{mM}$} & $-2.0 \pm 1.0$ & $-1.7 \pm 1.6$ & $-3.4 \pm 1.4$ & Not det. \\
\hline \multicolumn{2}{|c|}{$n=2+3, x^{*}$ isomer, $5 \mathrm{mM}$} & $-2.0 \pm 0.9$ & $-1.7 \pm 1.4$ & $-2.7 \pm 1.3$ & Not det. \\
\hline \multirow[t]{2}{*}{ (b) } & $n=3,4 \mathrm{mM}$ & $0.5 \pm 0.4$ & $0.3 \pm 0.3$ & $0.2 \pm 0.3$ & $-1.0 \pm 0.3$ \\
\hline & $n=5,40 \mathrm{mM}^{\mathrm{b}}$ & $-0.02 \pm 0.2$ & $-1.3 \pm 0.2$ & $-2.5 \pm 0.4$ & Not det. \\
\hline
\end{tabular}

${ }^{\text {a }}$ Estimated by extrapolation (see text).

${ }^{\mathrm{b}}$ Beyond the $25^{\circ} \mathrm{C}$ solubility limit.

Even though the molecules with $n=5$ and $2+3$ could be expected to have the same hydrophobicity, the substantial difference in the $\Delta G_{\text {tr }}$ suggests that the polarization of $\pi$ electrons is sensitive to the position of the phenyl ring in the bilayer.

As mentioned before, the $2 \leq n \leq 5$ alcohols coexist in the hydrocarbon and water moieties at $55^{\circ} \mathrm{C}$, thus the free energies of transfer are close to zero or are slightly negative (see Table 6). This is attributed to an aggregation between solute and surfactant molecules that provides similar stabilisation as the interaction with water. The radicals derived from $n=2+3$, however, are fully located within the lipid membrane at the same temperature (see Fig. 25).

With increasing solute size, i.e. increasing $n, \Delta G_{\text {tr }}$ decreases (Table 6), documenting the expected increasing hydrophobicity.

Furthermore, comparison of the results of the Gibbs free energy of water to bilayer transfer at various concentrations (Table $6 \mathrm{a}, \mathrm{b}$ ) confirms the concentration dependence of the phenyl alcohol partitioning.

The meta radical $n=3$ at $4 \mathrm{mM}$ concentration reveals much less favourable free energy values at $25-55^{\circ} \mathrm{C}$ than at higher concentration, as is shown in Tab. $6 \mathrm{a}, \mathrm{b}$. 
In the case of the more hydrophobic molecule $n=5$, increasing the solute concentration results in more negative $\Delta G_{\text {tr }}$ values, which means that the solute tends to saturate in the bilayer (at $40 \mathrm{mM}$ solute concentration we have five DHTAC molecules per solute molecule). These results are in agreement with those of Young and Dill for the bilayer/water partitioning of the hexane molecule into dimyristoylphosphatidylcholine vesicles (DMPC), where the partitioning coefficient decreases with hexane concentration at $25^{\circ} \mathrm{C}$ [89].

In mixed micelles, however, the effect of the concentration of added aliphatic alcohols on partitioning was observed by Verrall [109]. He found out that the long-chain alcohols at low concentration tend to solubilize in the palisade layer with the $\mathrm{OH}$ group anchored at the micelle surface between surfactant head groups, while at higher concentrations when the palisade layer is saturated, they solubilize in the micelle interior.

The concentration dependence of the partitioning of short chain alcohols (methanol to butanol) into the bilayer-water interface was also explored by Ly and Longo who related this effect to lowering of interfacial tension by alcohol molecules, which follows Traube's rule [77]. For each $\mathrm{CH}_{2}$ group in an alcohol, a three times lower alcohol concentration is required to reach the same interfacial tension. Furthermore, they found that the membrane permeability to these alcohols is also chain-length dependent due to more rapid adsorption as the chainlength increases.

Summarizing, it is clear that the phenyl alcohol partitioning is concentration dependent, although much less than in the case of aliphatic alcohols, therefore species containing a phenyl ring do not obey Traube's rule of transfer Gibbs free energy.

\section{The Hydrophobic Effect}

It is well accepted that the hydrophobic solvation of nonpolar solutes, such as aliphatic alcohols in water, is ascribed to the classical hydrophobic effect, which consists of two contributions: hydrophobic hydration and hydrophobic interaction [36,37,38]. The partitioning of the same species into the lipid bilayers is primarily driven by the nonclassical hydrophobic effect $[39,47]$.

The use of the ALC- $\mu$ SR technique has made it possible to explore the partitioning into the membrane of the series of phenyl alcohols even at very low concentrations. Its precise interpretation requires an understanding of the thermodynamic features that contribute to the free energy. Therefore, the enthalpy of transfer $\left(\Delta H_{\mathrm{tr}}\right)$ and the entropy of transfer $\left(\Delta S_{\mathrm{tr}}\right)$ 
relating to eqn. (4-2) were calculated from a linear fit of the Gibbs-Helmholtz equation to the $\Delta G_{\text {tr }}$ values in the temperature range between $25^{\circ} \mathrm{C}$ and $55^{\circ} \mathrm{C}$ :

$$
\Delta G_{\mathrm{tr}}=\Delta H_{\mathrm{tr}}-T \Delta S_{\mathrm{tr}}
$$

The values are collated for the five alcohols in Table 7, and the results are discussed here for the meta (and for $n=2+3$ for both) isomers. We consider the bilayer/water partitioning to be a two-step process, where the radical is first introduced into the aqueous solution, and subsequently it is expelled to the surfactant double layer. The aim of such a division is to show clearly how the classical hydrophobic equilibrium differs from the nonclassical one.

Table 7: Experimental values of enthalpy and entropy for phenyl alcohol transfer from the water into the bilayer at their $25^{\circ} \mathrm{C}$ solubility limit in the temperature range from 25 to $55^{\circ} \mathrm{C}$.

\begin{tabular}{|c|c|c|c|}
\hline \multicolumn{2}{|c|}{ Phenyl alcohol, concentration } & \multirow{2}{*}{$\frac{\Delta H_{\mathrm{tr}} / \mathrm{kJ} \mathrm{mol}^{-1}}{\text { Not det. }}$} & \multirow{2}{*}{$\frac{T \Delta S_{\mathrm{tr}} / \mathrm{kJ} \mathrm{mol}^{-1}}{\text { Not det. }}$} \\
\hline (a) & $n=2,40 \mathrm{mM}$ & & \\
\hline & $n=3,18 \mathrm{mM}$ & $47 \pm 7$ & $43 \pm 7$ \\
\hline & $n=4,16 \mathrm{mM}$ & $65 \pm 6$ & $61 \pm 6$ \\
\hline & $n=5,6 \mathrm{mM}$ & $22 \pm 7$ & $20 \pm 7$ \\
\hline \multicolumn{2}{|c|}{$n=2+3, y^{*}$ isomer, $5 \mathrm{mM}$} & $18 \pm 26$ & $20 \pm 25$ \\
\hline \multicolumn{2}{|c|}{$n=2+3, x^{*}$ isomer, $5 \mathrm{mM}$} & $6 \pm 23$ & $8 \pm 23$ \\
\hline \multirow[t]{2}{*}{ (b) } & $n=3,4 \mathrm{mM}$ & $17 \pm 5$ & $16 \pm 4$ \\
\hline & $n=5,40 \mathrm{mM}^{\mathrm{b}}$ & $37 \pm 6$ & $37 \pm 6$ \\
\hline
\end{tabular}

${ }^{\mathrm{a}}$ Estimated by extrapolation (see text).

${ }^{\mathrm{b}}$ Beyond the $25^{\circ} \mathrm{C}$ solubility limit.

\subsubsection{The Classical Hydrophobic Effect}

\section{Hydrophobic Hydration.}

According to theoretical work by Lee and Graziano [36,110] the hydration enthalpy of aliphatic alcohols is given by:

$$
\Delta H=E_{\mathrm{a}}+\Delta H^{\mathrm{h}}=E_{\mathrm{a}}(\text { H-bond })+E_{\mathrm{a}}(\mathrm{vdW})+\Delta H^{\mathrm{h}}
$$


where $E_{\mathrm{a}}$ is considered to be a sum of the direct alcohol-water H-bond energy $E_{\mathrm{a}}(\mathrm{H}-$ bond $)$ and the van der Waals interaction energy $E_{\mathrm{a}}(\mathrm{vdW}) . \Delta H^{\mathrm{h}}$ is the enthalpy contribution due to the reorganisation of $\mathrm{H}$-bonds of water molecules in the hydration shell.

The hydration entropy consists of the two following contributions:

$$
\Delta S=\Delta S_{\mathrm{x}}+\Delta S^{\mathrm{h}}
$$

where $\Delta S_{\mathrm{x}}$ relates to the entropy due to the excluded volume and $\Delta S^{\mathrm{h}}$ denotes the entropy in the reorganisation of the H-bonds which is compensated by $\Delta H^{\mathrm{h}}$ [36]. The introduction of the solute into water is associated with the work of cavity creation in the solvent, therefore Lee and Graziano assume that $\Delta S_{\mathrm{x}}$ can be calculated from the relation

$$
\Delta S_{\mathrm{x}}=-\Delta G_{\mathrm{c}} / \mathrm{T}
$$

where $\Delta G_{\mathrm{c}}$ corresponds to reversible work for cavity creation to accommodate the alcohol in the aqueous phase.

A similar procedure as for aliphatic alcohols, but excluding $\Delta H^{\mathrm{h}}$ and $\Delta S^{\mathrm{h}}$ contributions, is valid for aromatic rings. As reported by Graziano [111] the hydration enthalpy and entropy changes are

$$
\begin{gathered}
\Delta H=E_{\mathrm{a}}+\Delta H_{\mathrm{r}} \\
\Delta S=\Delta S_{\mathrm{x}}+\Delta S_{\mathrm{nx}}
\end{gathered}
$$

where $E_{\mathrm{a}}$ is the benzene-water dispersive interaction energy and $\Delta H_{\mathrm{r}}$ is the contribution to the solvent reorganisation caused by alcohol insertion. $\Delta S_{\mathrm{nx}}$ refers to the nonexcluded volume entropy contribution which is a compensating process at any temperature, thus $\Delta H_{\mathrm{r}}=T \Delta S_{\mathrm{nx}}$ [111].

Solute transfer from water into the bilayer.

On transfer of a solute from water to the bilayer the hydration terms are recovered so that they contribute to the transfer properties with opposite sign. Inspection of Table 7 reveals that $\Delta H_{\text {tr }}$ and $T \Delta S_{\text {tr }}$ are of similar magnitude in all considered cases, resulting in very small $\Delta G_{\text {tr }}$ values, which is not so for aliphatic alcohols that are transferred from water to the bilayer [39], probably because of their lower polarizabilities cavity work of the solute.

This suggests that the Gibbs free energy of transfer of the aliphatic part of the phenyl alcohol is compensated by an almost identical contribution from the aromatic part in both environments: 


$$
\begin{gathered}
\Delta G_{\mathrm{tr}}(\text { phenylalcohol })=\Delta G_{\mathrm{tr}}(\text { aliphatic part })+\Delta G_{\mathrm{tr}}(\text { aromatic part }) \\
\Delta G_{\mathrm{tr}}=\left[E_{\mathrm{a}}(\mathrm{H}-\text { bond })+E_{\mathrm{a}}(\mathrm{vdW})+\Delta G_{\mathrm{c}}\right]+\left[E_{\mathrm{a}}+\Delta G_{\mathrm{c}}\right]
\end{gathered}
$$$$
\Downarrow
$$$$
\Downarrow
$$

aliphatic part

aromatic part

These facts show that the free energy of transfer of the phenyl alcohol from the aqueous phase is balanced by the following contributions: the direct phenyl alcohol-water H-bond energy, the direct van der Waals interaction energy $\left(\Delta H_{\text {tr }}\right)$, and the excluded volume effect $\left(T \Delta S_{\text {tr }}\right)$ upon solute insertion into water. However, when the phenyl alcohol penetrates the membrane $\Delta H_{\text {tr }}$ is attributed to the direct phenyl alcohol-surfactant electrostatic interaction energy and the direct van der Waals interaction energy, while $T \Delta S_{\text {tr }}$ is affected by a binding of water molecules to the membrane surface and by changes in the bilayer organisation due to the alcohol penetration.

As described in section 4.1.3 the Gibbs free energies of water to bilayer solute transfer are small and positive (except at $55^{\circ} \mathrm{C}$ ) and decrease on increasing temperature. $\Delta H_{\text {tr }}$ and $T \Delta S_{\text {tr }}$ are both positive for the $3 \leq n \leq 4$ radicals. $\Delta H_{\text {tr }}$ increases by $18 \mathrm{~kJ} \mathrm{~mol}^{-1}$ per $\mathrm{CH}_{2}$ increment between $n=3$ and $n=4$ (Table 7a), but this may also depend on concentration. For the same alcohols the $T \Delta S_{\text {tr }}$ values increase by $18 \mathrm{~kJ} \mathrm{~mol}^{-1}$ increments. This demonstrates that the positive entropy of hydration of the hydrophobic solute is recovered. Based on $\Delta G_{\text {tr }}$ for the $n$ $=2$ species we would expect also positive $\Delta H_{\text {tr }}$ and $T \Delta S_{\text {tr }}$ values, although smaller than for $n=$ 3 due to its lower hydrophobicity, and thus lower affinity to the nonaqueous regime.

In the classical model for the transfer of alcohols from water to pure alcohols the entropy change upon partitioning arises from the decrease in entropy in the water phase when the hydrocarbon part is removed from it, which is proportional to the size of such a nonpolar part [39].

It is important to remember that the values of $\Delta H_{\text {tr }}$ and $T \Delta S_{\text {tr }}$ obtained by ALC- $\mu$ SR are temperature averaged values over $25-55^{\circ} \mathrm{C} . \Delta H_{\text {tr }}$ is also affected by the gel to liquid crystal phase transition (the onset of which is at ca. $55{ }^{\circ} \mathrm{C}$ ) that is estimated to be $2 \mathrm{~kJ} \mathrm{~mol}^{-1}$ per $\mathrm{CH}_{2}$ group of the surfactant chain [46].

The above facts indicate that the transfer of $2 \leq n \leq 4$ phenyl alcohols into the bilayer follows the classical hydrophobic effect. 


\section{Hydrophobic Interaction.}

From the reason of the very low concentration of all phenyl alcohols $(5-40 \mathrm{mM})$ in the present experiment we conclude that bulk hydrophobic interaction does not occur. Furthermore, it is known that in solutions more dilute than $<1.1 \mathrm{M}$, the alcohols do not show any tendency to aggregate in water [35]. There is also no chemical reaction that could cause an enforced hydrophobic interaction.

Figure 30 demonstrates that the positions of the $\Delta_{0}$ resonances of the bulk $n=5$ alcohol correspond to the positions of the $\Delta_{0}$ lines in the surfactant part $\left(\mathrm{m}^{*}\right)$ of the $40 \mathrm{mM}$ surfactant emulsion (i.e. beyond the $25{ }^{\circ} \mathrm{C}$ solubility limit). It suggests that the radicals do not aggregate in water, forming a separate phase of the pure alcohol, but the fraction that could not be dissolved in water migrated into the bilayer and aggregated with DHTAC amphiphiles.

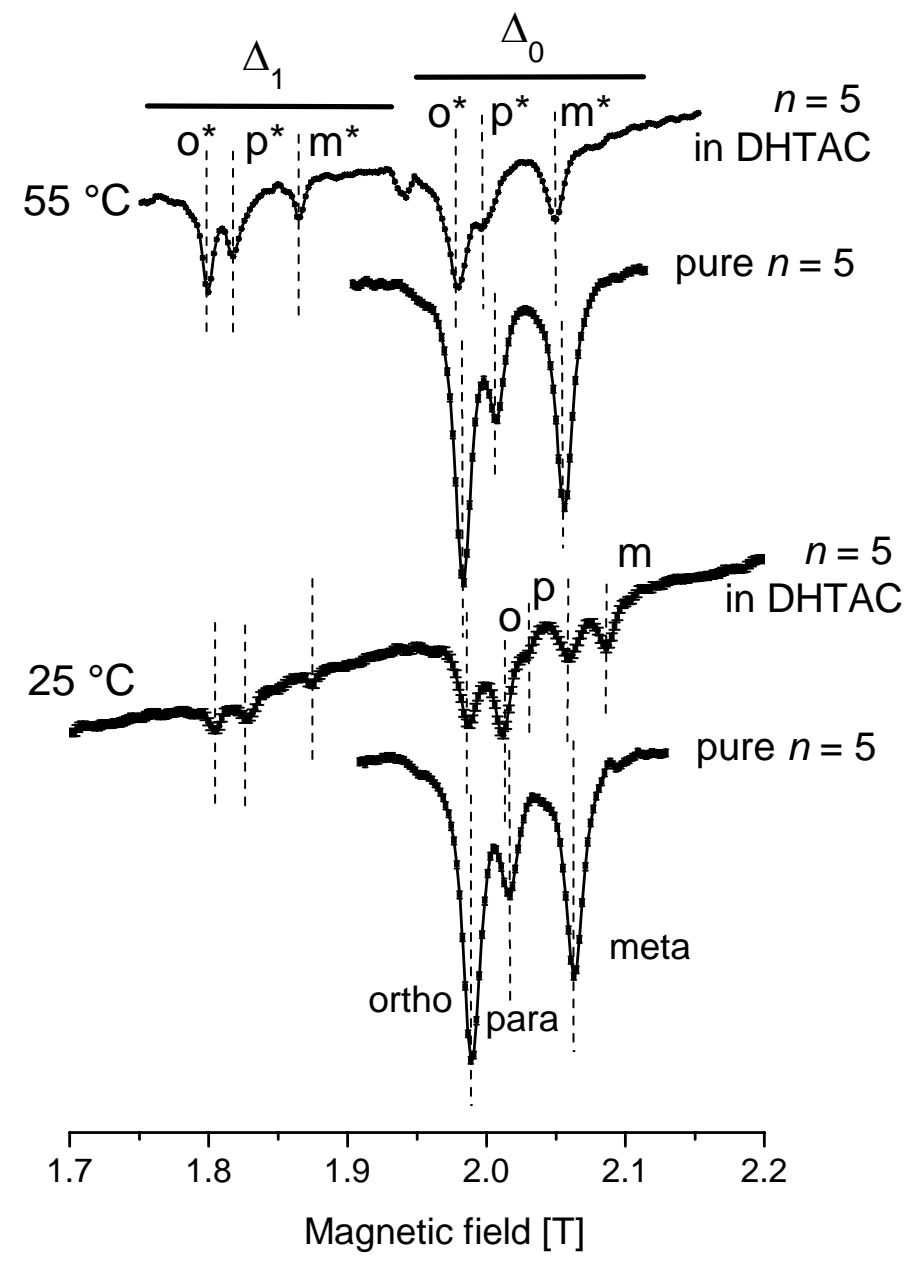

Figure 30: Methylene $\Delta_{0}$ resonances of the three isomers of the $\mathrm{Mu}$ adduct to 5phenylpentan-1-ol in bulk liquid alcohol and in $15 \%$ aq. DHTAC at $55{ }^{\circ} \mathrm{C}$ (top) and at $25{ }^{\circ} \mathrm{C}$ (bottom). 


\subsubsection{The Nonclassical Hydrophobic Effect}

A negative increment of the enthalpy and entropy of transfer per additional methylene group is characteristic of partitioning into the membrane [39,46,47].

The thermodynamic data summarised in Table 7 indicate that in contrast to the $2 \leq n \leq 4$ phenyl alcohols, $\Delta H_{\text {tr }}$ and $T \Delta S_{\text {tr }}$ for $n=5$ unexpectedly decrease. Similar behaviour is revealed by the $n=2+3$ radical, even within the errors, because we expect a negative sign for both thermodynamic features due to its $\Delta G_{\mathrm{tr}}<0$, which means that a significant fraction of this alcohol penetrates the bilayer (see Figure 25).

Obviously, the large decrease in $\Delta H_{\text {tr }}$ values of the $n=5$ radical reveals a deviation from the classical interpretation and suggests that the longer-chain alcohols enhance the lipid-lipid interactions and participate in them. The sign of $\Delta H_{\text {tr }}$ still remains positive due to the presence of a certain fraction of these alcohols in the aqueous region.

The sudden decrease in the magnitude of $T \Delta S_{\text {tr }}$ values for longer-chain alcohols is probably attributed to a loss of configurational entropy of the bilayer phase due to the binding of the alcohol to the surfactant membrane and upon the exposure of lipid moieties to water in the interfacial region.

Rowe and co-workers [39] using titration calorimetry, found that n-alcohol penetration into dipalmitoylphosphatidylcholine (DPPC) multilamellar vesicles is driven by the enthalpy, which decreases by $7.4 \mathrm{~kJ} \mathrm{~mol}^{-1}$ per $\mathrm{CH}_{2}$ group (roughly a factor of three less than derived here for the phenyl alcohols) due to the bilayer effect. They observed that the sign of the $\Delta H_{\text {tr }}$ changes from positive for hexanol to negative for heptanol-nonanol, which suggest that the smaller alcohols disrupt interactions in the bilayer, while the longer one participate in the lipid-lipid interaction. Furthermore, Seelig and Ganz [47] showed that the water-bilayer transfer of four charged amphiphiles is dominated by the van der Waals interaction between the nonpolar residues of the solute and the hydrophobic core of the lipid membrane. This was attributed to the nonclassical hydrophobic binding.

We believe that our experimental results presented above and their careful explanation reflect that the transfer of the series of phenyl alcohols from water to DHTAC is a combination of the classical and nonclassical hydrophobic effects which depend on temperature and chain length. 


\subsubsection{Information from Spin Exchange Experiments with $\mathrm{Ni}^{2+}$}

Finally, the question arises whether the guest probe molecule is incorporated in or adsorbed at the water/surfactant interface or perhaps exchanges rapidly between the two moieties.

The Heisenberg spin exchange reaction between the muoniated radicals and paramagnetic $\mathrm{Ni}^{2+}$ cations was studied to answer this question. It has previously been demonstrated that chromium oxalate as relaxation enhancer penetrates into lipid membranes and reaches at low concentrations the hydrocarbon moiety [100]. On this basis we expect that $\mathrm{Ni}^{2+}$ ions does also not partition significantly into the bilayers and that it should affect primarily the signals of the substituted cyclohexadienyl radicals in the aqueous regime but not in the bilayer.

Therefore, as it is shown below, ALC- $\mu$ SR permits the determination of the spin exchange rate constant from the broadening of the $\Delta_{0}$ resonances, which provides a second means for determining to the preferred location of the probe molecules.

\subsubsection{Determination of the Spin Exchange Rate Constant}

A quantitative numerical analysis of the effect of spin exchange (in combination with chemical reaction) was developed by Heming et al. [112]. Kreitzman developed an analytical model based on the Liouville formalism for the isotropic case, where only $\Delta_{0}$ (muon-proton spin flip-flop transitions, conserving the total magnetic quantum number $M$ ) resonances appear [113].

In the absence of $\mathrm{NiCl}_{2}$, all ALC resonances are narrow and well separated, although the lines which belong to the meta isomer $\left(\mathrm{m}^{*}, \mathrm{~m}\right)$ are the best distinguished (see Figure 31 ), thus the results are discussed here for the meta species of the muoniated 2-phenylethan-1-ol in the lamellar DHTAC dispersions.

The presence of the paramagnetic $\mathrm{Ni}^{2+}$ ions in the aqueous region of a DHTAC/water emulsion leads to a broadening of the ALC resonances if the muoniated radical also resides in the aqueous phase and therefore undergoes Heisenberg spin exchange. In consequence, the $\Delta_{0}$ lines that arise from the meta isomer in water $(\mathrm{m})$ broaden with increasing nickel concentration, while the ones in the surfactant membrane $\left(\mathrm{m}^{*}\right)$ are almost unchanged as demonstrated in Figure 31. 
The electron spin flip rate $\lambda_{\mathrm{e}}$ is obtained from global fits of the $\Delta_{0}$ resonances over all spectra with different $\mathrm{Ni}^{2+}$ concentrations.

$\lambda_{\mathrm{e}}$ is related to the spin exchange rate constant $k$ via

$$
\lambda_{\mathrm{e}}=\lambda_{0}+k\left[\mathrm{Ni}^{2+}\right]
$$

where $\left[\mathrm{Ni}^{2+}\right]$ denotes the concentration of nickel ions and $\lambda_{0}$ a self-relaxation of the muon spin that also occurs in the absence of any paramagnetic species.

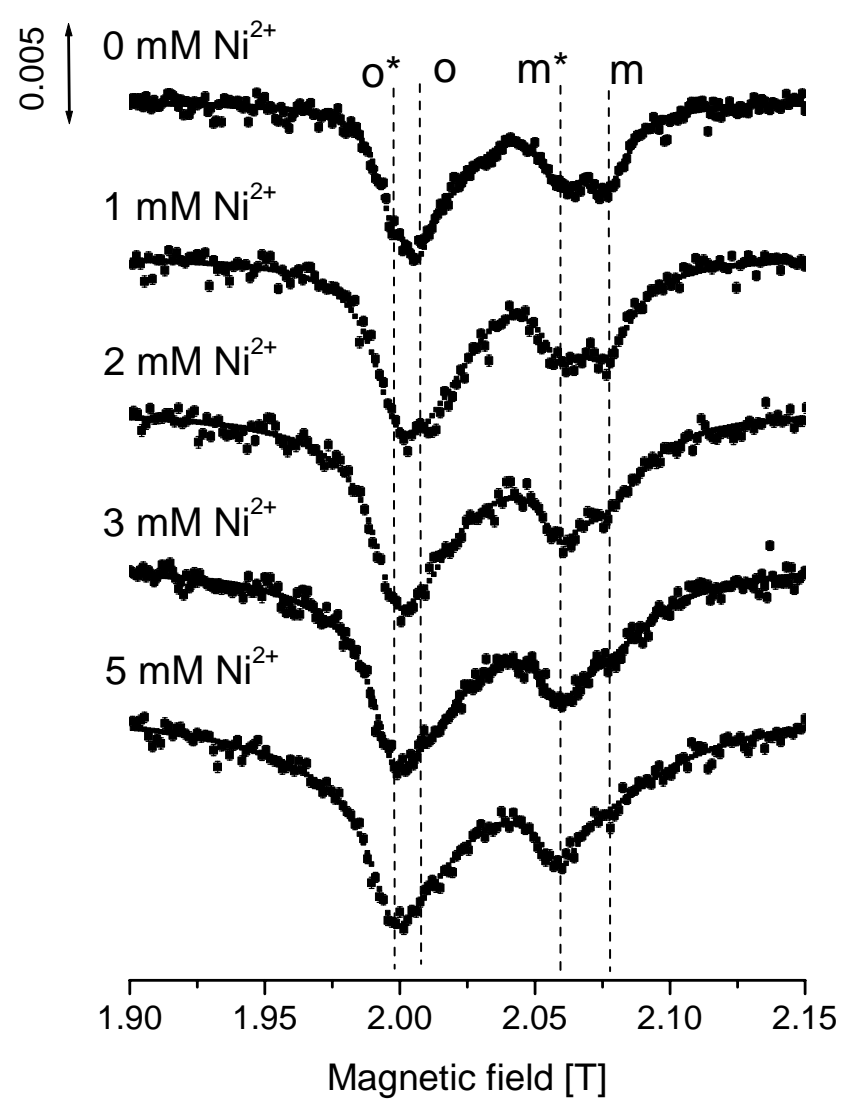

Figure 31: ALC- $\mu \mathrm{SR}$ spectra of $40 \mathrm{mM}$ muoniated 2-phenylethan-1-ol in DHTAC/water dispersion with different $\mathrm{Ni}^{2+}$ concentrations at $55^{\circ} \mathrm{C}$.

Taking into account an additional relaxation, the muon spin flip rate is taken as $\lambda_{\mu}=1 / \tau_{\mu}+\lambda_{0}$, where $\tau_{\mu}$ is the muon lifetime. Details about the determination of $k$ were reported elsewhere [26]. The results are given in Table 8 and will be discussed in detail in section 4.1.4.2.

At a field of $2 \mathrm{~T} \lambda_{0}$ is very small [114] and because of its independence on the $\mathrm{Ni}^{2+}$ concentration of no further interest here. However, residual oxygen will have a similar effect 
as nickel at the same concentration. Therefore, all oxygen had to be removed from the solvent by bubbling it with pure nitrogen for more than one hour.

To remove the oxygen from the surfactant, the pure material was melted and solidified several times under a continuous nitrogen flow. The latter procedure is particularly crucial since the solubility of molecular oxygen is significantly higher in the hydrocarbon moiety of the membrane than in water [100].

Table 8: Spin exchange rate constants for the meta isomer of the $n=2$ radical in water and in $15 \%$ aq. DHTAC solution.

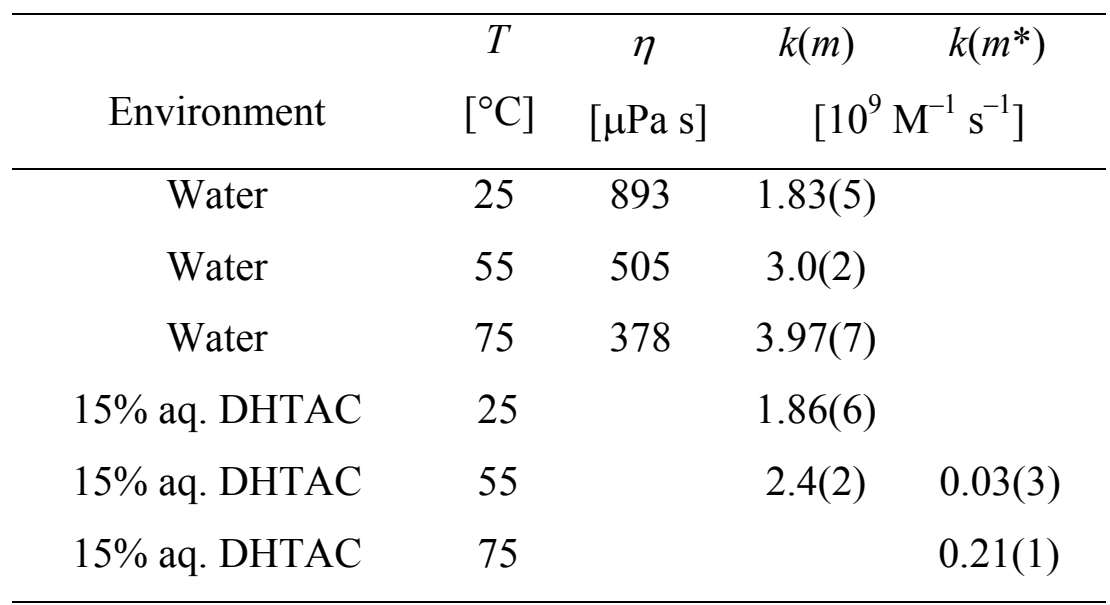

\subsubsection{Dynamic Phenomena}

The Heisenberg spin exchange rate of the muoniated 2-phenylethyl-cyclohexadienyl radical with paramagnetic $\mathrm{Ni}^{2+}$ cations at $25^{\circ} \mathrm{C}$ and $75{ }^{\circ} \mathrm{C}$ was investigated in recent work [86].

In the $15 \%$ aqueous DHTAC emulsion, the spin exchange is 9 times slower at $75{ }^{\circ} \mathrm{C}$ than at $25{ }^{\circ} \mathrm{C}$ despite the lower viscosity at the higher temperature. This is attributed to a very low nickel concentration in the $\mathrm{L}_{\alpha}$ phase. The cations do not have access to the surfactant membrane where the radical is located. In the aqueous phase, however, with increasing temperature the spin exchange becomes faster due to a decrease of the water viscosity, as is shown in Table 8 . From the ratio $k_{\mathrm{m}^{*}} / k_{\mathrm{m}}=0.21 / 3.97=0.05$ it was concluded [86] that in the high temperature $\mathrm{L}_{\alpha}$ phase the alcohol molecules resides mostly in the DHTAC membrane, at $55 \%$ aqueous character (Figure 28). The residual spin exchange rate may be due to a limited 
access of the nickel ions to the head group regime of the bilayer or to a rapid exchange of the alcohol molecule between the two environments with predominant residence in the membrane.

At an intermediate temperature of $55^{\circ} \mathrm{C}$, near the $\mathrm{L}_{\alpha} \leftrightarrow \mathrm{L}_{\beta}$ phase transition, the meta isomer shows a split signal. Simultaneously, Figure 28 reveals 55\% and 93\% aqueous character [98]. These are clear evidences for the coexistence of 2-phenylethan-1-ol in the nonaqueous/aqueous environment at this temperature. However, what kind of coexistence is it? The above facts indicate that a fraction of the $n=2$ radicals still remains in the surfactant bilayer, as shown by the presence of the $\Delta_{1}$ lines and $m^{*}$ (Figure 31 ). It is important to note that when the $n=2$ radical is incorporated within the surfactant membrane the spin exchange rate constant is of similar magnitude as in the higher temperature (Tab. 8). In contrast to other phenyl alcohols, $n=2$ reveals the same polarity at $55{ }^{\circ} \mathrm{C}$ and $75{ }^{\circ} \mathrm{C}$ (see Figure 28), suggesting that the radical is anchored between DHTAC amphiphiles and does not exchange between aqueous/nonaqueous regions. Moreover, at an intermediate temperature of $55{ }^{\circ} \mathrm{C}$ we have coexistence of the $\mathrm{L}_{\alpha}$ and $\mathrm{L}_{\beta}$ phases, and therefore of the high and low temperature behaviour, where particular regions of the surfactant film are still easy to penetrate.

The other fraction, however, is squeezed out from the membrane into the water phase, as demonstrated by the split $\Delta_{0}$ resonance of the meta isomer $\left(m, m^{*}\right)$ in Figure 31 . In the $15 \%$ aq. DHTAC the rate constant is slightly larger at $55{ }^{\circ} \mathrm{C}$ than at $25{ }^{\circ} \mathrm{C}$ upon the higher viscosity of water contained in the surfactant solution. Furthermore, the aqueous character sensed by the ortho and meta isomers deviates clearly more from the expected $100 \%$ than for the higher alcohols. A key point is that spin exchange in bulk water is faster by about $20 \%$ than in DHTAC dispersion as it was expected. This means that this fraction of the alcohol is located closer to the hydrocarbon/water interface (93\% aq.). The lower value of the rate constant can be explained by the slower exchange of the alcohol between two environments, due to the repulsion between positive trimethylammonium head groups of the surfactant and nickel cations.

At low temperature $\left(25-35^{\circ} \mathrm{C}\right)$ the $n=2$ alcohol shows still a behaviour that is distinctly different from the others: it is the only one that does not exhibit split resonances which are indicative of coexistence. Based on the extrapolated $\Delta G_{\mathrm{tr}}$ value of ca. $4.9 \mathrm{~kJ} \mathrm{~mol}^{-1}$ at $25^{\circ} \mathrm{C}$ we would expect to find $88 \%$ of the alcohol in water and $12 \%$ in the bilayer. If present this latter fraction should have led to observable signals [84]. The explanation is rapid exchange with $88 \%$ probability in water (100\% aq.) and $12 \%$ in the bilayer (at ca. $60 \%$ aq.). The weighted 
average of these numbers amounts to $95 \%$ aqueous character, which is close to the observed value of ca. $93 \%$. We therefore conclude that in the $\mathrm{L}_{\beta}$ phase the shortest alcohol, and thus the best soluble (solubility $20 \mathrm{~g} / 1$ at $20^{\circ} \mathrm{C}$ ) [115] exchanges rapidly on the $10^{-5} \mathrm{~s}$ timescale (see Section 4.1.3), while the others certainly do not exchange at this rate. For comparison, it should be noted that the timescale of exit of benzyl tert-butyl nitroxide radicals from SDS, HTAB and $\mathrm{C}_{10} \mathrm{E}_{6}$ spherical micelles was reported to be ca. $2.5 \times 10^{-7} \mathrm{~s}$ at room temperature [116]. It is of course conceivable that the bilayers are somewhat more tightly packed in the $\mathrm{L}_{\beta}$ phase than the spherical micelles, so that kinetics would slow down.

The spin exchange rate with $\mathrm{Ni}^{2+}$ is within error the same in the presence of $15 \%$ DHTAC (from which $\mathrm{Ni}^{2+}$ is expelled) as in pure water. From this finding we conclude that the radical spends ca. $100 \%$ instead of the above $88 \%$ of its time in water and very little in the DHTAC bilayer.

Our results and their interpretation are consistent with those of Brigati [116] and Verrall [109]. They reported that within a series of solutes containing a different number of aromatics rings or different alkyl substituents, the exit rates decrease rapidly with decreasing water solubility. In other words, the value of the exit rate depends directly on the probe hydrocarbon chain length. An increase in the alcohol chain length increases the hydrophobic interactions, leading to increased stabilisation of the alcohol in the surfactant membrane and a reduction in the exit rate of the alcohol. More specifically, the $\mathrm{OH}$ group of the alcohol locates in the micelle or bilayer head group region, whereas its hydrophobic tail embeds itself in the interior of the lipid layer interacting hydrophobically with the hydrocarbon chains of the surfactant. Moreover, the changes in the intermolecular forces in the surfactant head group region due to the replacement of the water molecules by the hydrophilic moiety of the alcohol appear to have no net effect [109].

\subsubsection{Reorientational Dynamics of $\mathrm{MuC}_{6} \mathrm{H}_{5}\left(\mathrm{CH}_{2}\right)_{3} \mathrm{OH}$}

As shown in Subsection 2.3.3, any reorientational motion of the muoniated cyclohexadienyl radical averages partly or completely its hyperfine anisotropy. It is well known that the dynamics of the radical strongly depends on the size and shape of the considered molecule, the type of the surrounding environment and its temperature. 
Presented in Figure 32, the dynamics of the Mu adduct to 3-phenylpropan-1-ol in the DHTAC emulsion at $75^{\circ} \mathrm{C}$, is considered here as an example.

(a)

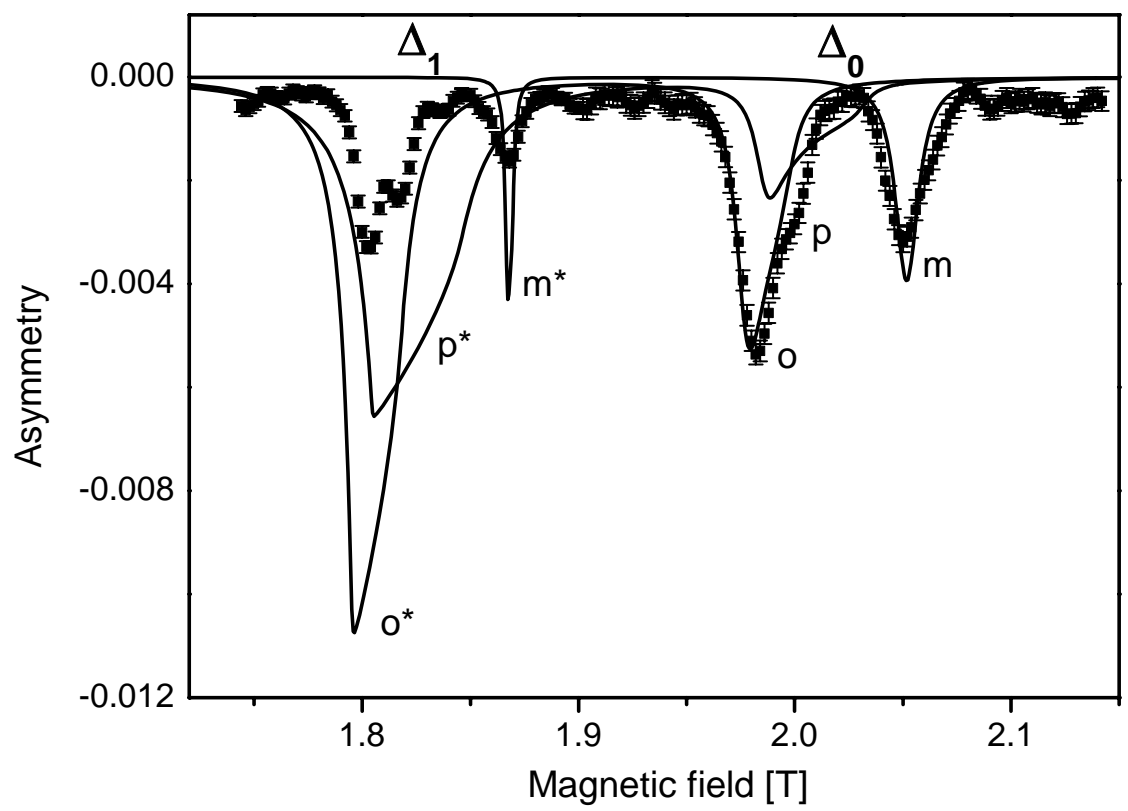

(b)
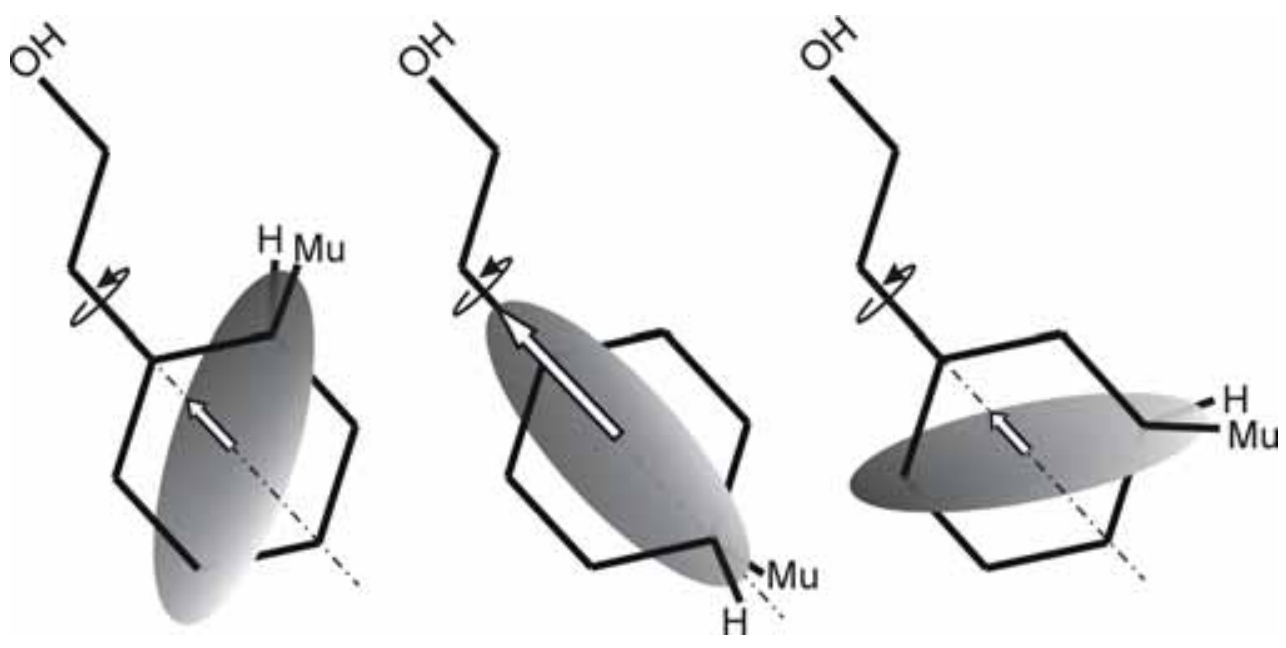

Figure 32: (a) Experimental $\Delta_{1}$ and $\Delta_{0}$ resonances of the $\mathrm{Mu}$ adduct to phenylpropanol obtained at $75{ }^{\circ} \mathrm{C}$ (squares) and simulated ALC- $\mu$ SR spectra of the three isomers assuming the radicals are rotating about the first substituent bond without any further reduction in hyperfine anisotropy (solid lines). (b) Methylene muon dipolar hyperfine tensor represented by an ellipsoid for the $\mathrm{Mu}$ adducts in the ortho, para and meta positions of the alkanol substituent. The arrow shows the component that is retained when the ring rotates about the first substituent bond. 
At this temperature the molecule is incorporated within the lipid membrane, which is confirmed by the presence of the $\Delta_{1}$ resonances.

The limited available space in the bilayer and the near-linear geometry of the solute restrict the radical dynamics; we therefore assume that $\mathrm{MuC}_{6} \mathrm{H}_{5}\left(\mathrm{CH}_{2}\right)_{3} \mathrm{OH}$ can undergo only uniaxial rotation about the first $\mathrm{C}-\mathrm{C}$ substituent bond at the phenyl ring (Figure 32b), and we compare the experimental with simulated line shapes (Figure 32a).

The amplitudes of the simulations are scaled to the experimental $\Delta_{0}$ resonances, which corresponds to a scaling to the radical chemical yield. The static proton hyperfine anisotropies are obtained from $a b$ initio calculations, performed with Gaussian 98 [117] using the UB3LYP method with the 6-311G basis set, and the corresponding muon values were scaled by the ratio of the muon and proton magnetic moments. The components of the theoretical dipolar muon and proton hyperfine coupling components parallel to this rotation axis $\left(D_{\|}^{\mu, t h e o}\right.$ and $D_{\|}^{\text {ptheo }}$, respectively) were calculated as reported by Weil, Wertz and Bolton [118]. The dipolar hyperfine tensor $\boldsymbol{D}^{\boldsymbol{\mu}}$ and the component $D_{\|}^{\mu}$ that is invariant under rotation are displayed schematically in Figure 32b. It is seen that rotation about the given axis leads to a new parallel component (arrow), which is considerably reduced for the ortho and meta isomers while nearly the maximum value is retained for the para isomer. For sufficiently small values of this effective hyperfine anisotropy this results in an extensive reduction of the ortho and meta $\Delta_{1}$ signal amplitudes [108].

For axial systems $D_{\|}^{\text {theo }}$ is given by $D_{\|}^{\text {theo }}=A_{\|}-A_{0}$, and because $\boldsymbol{D}$ is traceless, $D_{\perp}^{\text {theo }}=-D_{\|}^{\text {theo }} / 2$. The experimental and calculated uniaxial dipolar hyperfine coupling components of the $\mathrm{Mu}$ adduct to 3-phenylpropanol for pure uniaxial rotation about the first substituent bond are presented in Table 9.

The simulated $\Delta_{0}$ lines of the ortho and meta isomers fit the experimental ones very well, whereas the experimental peak of the para species is narrower than the simulation (see Figure 32a). Moreover, the experimental $\Delta_{0}$ resonance of the para isomer is the weakest among all isomers as expected from the number of Mu addition sites on radical formation.

Further inspection of Figure $32 \mathrm{a}$ reveals that the experimental $\Delta_{1}$ resonances are of Lorentzian shape. The peaks of the ortho and para isomers are much narrower and of lower intensity than the simulated peaks. This is attributed to more extensive averaging of the anisotropy than given by uniaxial rotation. 
Table 9: Parallel uniaxial hyperfine coupling components of the $\mathrm{Mu}$ adduct to 3phenylpropanol: experimental $\left(D_{\|}^{\mu, e x p}\right)$ and calculated values for pure uniaxial rotation about the first substituent bond ( $D_{\|}^{\mu \text { theo }}$ and $D_{\|}^{\text {p.theo }}$, see text).

\begin{tabular}{cccc}
\hline & $D_{\|}^{\mu, \text { exp }} / \mathrm{MHz}$ & $D_{\|}^{\mu \text { theo }} / \mathrm{MHz}$ & $D_{\|}^{\text {t,theo }} / \mathrm{MHz}$ \\
\hline ortho & $0.6(2)$ & 3.9 & 1.3 \\
para & $1.2(3)$ & 7.1 & 1.4 \\
meta & $0.6(2)$ & 0.56 & 0.06 \\
\hline
\end{tabular}

Experimental values of the parallel component $\left(D_{\|}^{\mu, e x p}\right)$ of the effective uniaxial hyperfine anisotropy were evaluated by fitting the $\Delta_{1}$ signals. The ratio between $D_{\|}^{\mu, e x p}$ (para) and $D_{\|}^{\mu, e x p}$ (ortho) is ca. 2:1 (see Table 9). This relative value agrees with the simulation, but the absolute effective $D_{\|}^{\mu, e x p}$ values of the ortho and the para resonances are smaller by roughly a factor of six than the corresponding $D_{\|}^{\mu, t h e o}$ values, which suggests that the rotation axis itself undergoes fast and extensive further motion, such as wobbling or tumbling. Due to the limited space among the DHTAC amphiphiles such a motion is not isotropic, and the reduction factor is perhaps best described as the analogue of an order parameter in a liquid crystal. The observations are similar to those of Roduner and co-workers for the reorientational dynamics of the cyclohexadienyl radical in high-silica ZSM-5 zeolite. They found a main rotation axis that is perpendicular to the molecular plane. A two-site jump process leads to a $D_{\|}^{\mu, e x p}$ value of $-1.6 \mathrm{MHz}$, which is smaller by a factor of four than the simulated $D_{\|}^{\mu, t h e o}(-6.8 \mathrm{MHz})$ for the static case [23].

The weakest $\Delta_{1}$ resonance is that of the meta species, in agreement with the small predicted muon hyperfine anisotropy, $D_{\|}^{\mu, t h e o}$, for pure uniaxial rotation about the first substituent bond in the meta position, as given in Table 9 (see also Figure 32b). The $D_{\|}^{\mu, e x p}$ component of the meta radical, being already at very low level, experiences no further loss of anisotropy.

Many lyotropic liquid crystals can be aligned in sufficiently high applied magnetic fields, usually on the order of several Tesla. This alignment depends on various factors such as viscosity, elasticity, composition of the phases, temperature $[119,120]$. In principle, this effect 
can contribute to the narrowing or even disappearance of the $\Delta_{1}$ resonance, but nothing is known about the sensitivity of the present system to magnetic fields, and the effect remains subject to further investigation.

\subsubsection{Conclusions}

In applications via the technique of $\mathrm{ALC}-\mu \mathrm{SR}$, the positive muon has been used to study the partitioning of series of phenyl alcohols $2 \leq n \leq 5$ in the lamellar phase colloidal dispersions of a cationic dichain surfactant, DHTAC.

The position of one type of resonance, which on the basis of spectroscopic selection rules is denoted as $\Delta_{0}$, is related to the solvent polarity of the radicals' environment. The results derived from $\Delta_{0}$ measurements reveal a systematic trend where the increasing chain length of the phenyl alcohol results in a deeper immersion of the phenyl ring of the alcohol into the surfactant bilayer with the $\mathrm{OH}$ group anchored at the interface. The aqueous character of the para isomer deviates significantly from that of the ortho and meta species. In addition, the data suggest partial penetration of water molecules into the bilayer.

Furthermore, data ensuing from a second resonance (called $\Delta_{1}$, which is dependent upon the degree of confinement of the radical within the surfactant aggregate structure) indicates not only that the phenyl alcohol resides in an anisotropic environment, (i.e. that the host molecule is unable to undergo full 3-dimensional re-orientation on a timescale of $50 \mathrm{~ns}$ ), but the resonance line widths indicate that the radicals are undergoing fast rotation about a particular axis, in this instance about the first $\mathrm{C}-\mathrm{C}$ substituent bond at the phenyl ring. Detailed analysis of these $\Delta_{1}$ line shapes suggests that other types of motion involving reorientation of the above rotation axis are also present.

At room temperature, the hydrocarbon chains of the DHTAC double layers form an aggregate state commonly referred to as the $\mathrm{L}_{\beta}$ phase, where the motions of surfactant alkyl chains are effectively frozen out. These chains melt on heating over a temperature range which is solution composition dependent (ca. 51 to $67^{\circ} \mathrm{C}$ ), but in all cases leading to a liquid-like disordered hydrocarbon regime whilst retaining the overall lamellar structure (and in this state is termed $\mathrm{L}_{\alpha}$ ). Above the $\mathrm{L}_{\alpha} / \mathrm{L}_{\beta}$ chain ordering phase transition the tracer molecules reside within the bilayer, but below this transition (and depending on their water-oil solubility) they are completely or partly expelled. 
This interpretation is further supported by Heisenberg spin exchange experiments, indicating simultaneously, that a fraction of the shortest probe molecule exchange rapidly between aqueous/nonaqueous environment in the low temperature $\mathrm{L}_{\beta}$ phase, which is not so for higher phenyl alcohols.

The bilayer-water partitioning reflects both typical classical and nonclassical hydrophobic solvation depending on temperature, chain length, concentration and structure of phenyl alcohols.

Finally, the ALC- $\mu$ SR revealed that the distribution of the probe molecules containing phenyl rings deviate from Traube's rule that is operative for the aliphatic alcohols. 


\subsection{Ternary and Quaternary Systems of SDS}

The partitioning of a series of phenyl alcohols in the lamellar phase systems of a dichain cationic surfactant, which serves as a model compound for the phospholipid constituent of cell membranes, was shown in the previous Chapter. The challenge of this part of the thesis is to elucidate the affinity of two probe molecules for the aqueous and nonaqueous environments in more complex dispersions than examined previously. We focus on the comparison of the partitioning and the reorientational dynamics of 3-phenylpropan-1-ol (the more hydrophilic molecule) with 5-phenylpentan-1-ol (the more hydrophobic molecule) in ternary sodium dodecyl sulfate (SDS)/water/pentanol mixtures and in quaternary systems comprising SDS, water, pentanol and dodecane.

The normal micellar $\mathrm{L}_{1}{ }^{1}$, lamellar $\mathrm{D}^{4}$, rectangular $\mathrm{A}^{8,9}$ and hexagonal phase $\mathrm{E}^{10}$ structures were obtained at $25{ }^{\circ} \mathrm{C}$ and ambient pressure by changing the composition, as shown in the phase diagram of Figure 33 and in Table 5 (see Subsection 3.1.3). Several phases in this system have an inverse structure due to the high dilution in oil, as in water-in-oil microemulsions $\mu E^{2,3}$, the reverse lamellar $\mathrm{D}^{5}$, hexagonal $\mathrm{F}^{11}$ and sponge phase $\mathrm{L}_{3}{ }^{6,7}$. These structures have different curvatures of the water-surfactant interface. In all cases (except for the spherical micelles and microemulsions), the basic structural unit is a bilayer that is composed of two roughly parallel and oppositely oriented SDS monolayers, separated by water. The pentanol molecules partition into the bilayer, adding flexibility and modulating its spontaneous curvature. In the ternary systems electrostatics is the dominant factor in producing stability and relative rigidity [121], whereas the quaternary systems are stabilized by Helfrich undulation forces which arise from thermal fluctuations of the flexible layers as an effect of dilution with 'oil' (dodecane) [122,123]. The detailed description of structural alignment of particular SDS mesophases has been given elsewhere [52,66,124].

The two basic questions that arise in context of this work are whether the intercalation of the phenyl alcohol probe molecules depends on the curvature of the water-surfactant interface and whether the thickness of the 'oil' phase has any influence on the probe molecule distribution. Furthermore, it is interesting to explore the differences and similarities of the alcohol location in the lamellar phase of the present anionic single chain SDS surfactant and the cationic dichain surfactant DHTAC that was presented in the previous Section 4.1. 


\subsubsection{Identification of SDS Mesophases}

Optical polarizing microscopy investigations revealed characteristic morphologies of the SDS based mesophases containing phenyl alcohols as shown in Figure 33.

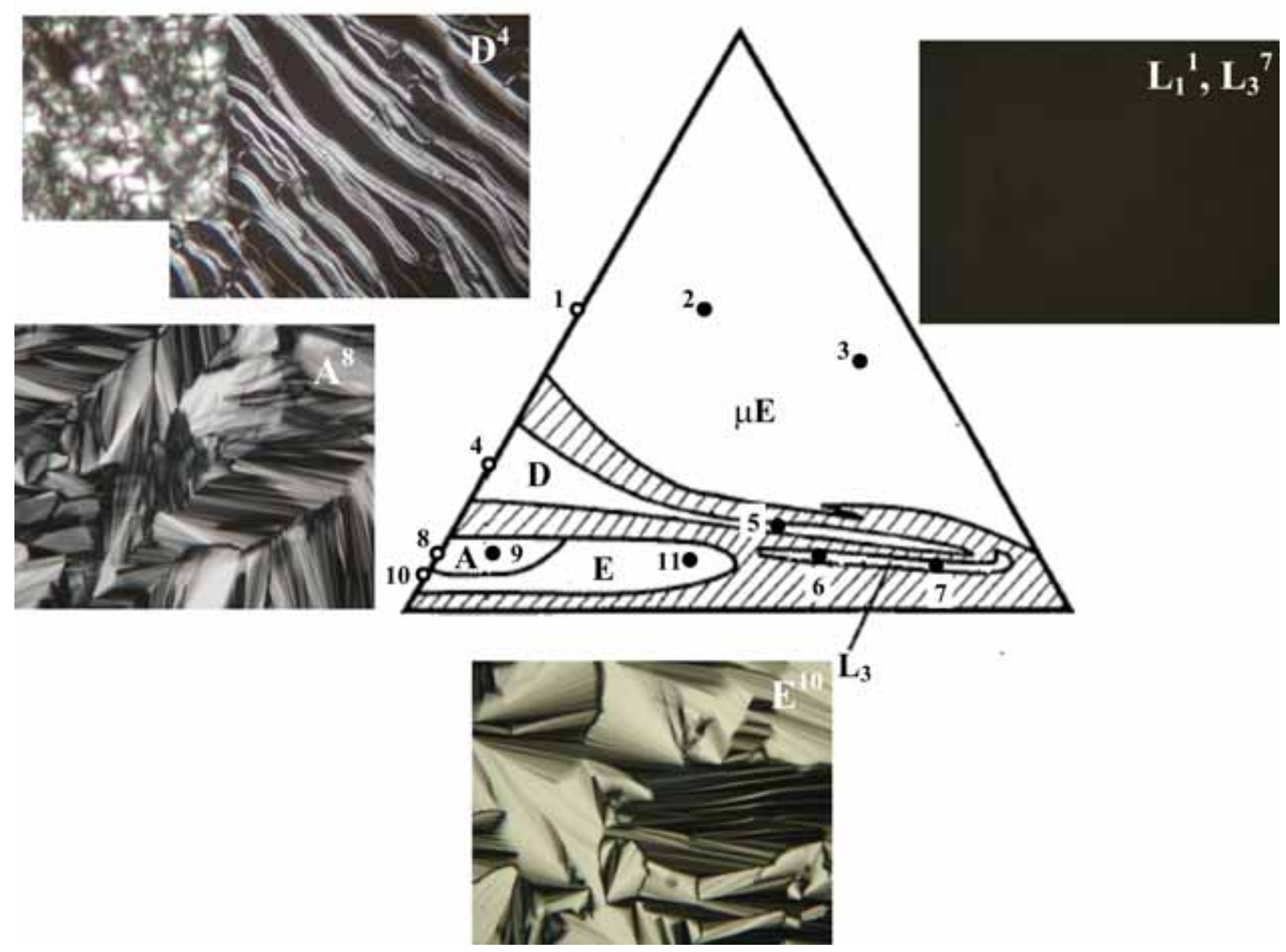

Figure 33: The partial triangular phase diagram of the quaternary SDS, water, pentanol, dodecane system at $25{ }^{\circ} \mathrm{C}$ [124], with corresponding textures of the SDS mesophases comprising phenyl alcohols in polarized light microscopy. The water-to-surfactant weight ratio $(\mathrm{W} / \mathrm{S}=1.552 \pm 0.002)$ is kept constant. $\mu \mathrm{E}$ is the microemulsion, $\mathrm{D}$ is the lamellar phase, $\mathrm{L}_{3}$ is the sponge phase, $\mathrm{A}$ is the rectangular phase and $\mathrm{E}$ is the hexagonal phase. The numbered dots indicate the mixtures studied, and open circles stand for mixtures without dodecane while filled dots represent samples with dodecane. In the text they are given as superscripts to the phase symbol. Dashed areas refer to the coexistence of different phases.

The micellar $\mathrm{L}_{1}{ }^{1}$ and sponge $\mathrm{L}_{3}{ }^{7}$ (the superscripts refer to the actual composition as given in Figure 33 and Table 5) structures were optically isotropic, giving a black texture. However, in the sample $\mathrm{L}_{3}{ }^{6}$ of the sponge phase very small birefringent regions appeared, while the rest of the sample was optically isotropic. The same phenomenon was observed by Magalhaes 
[52] and co-workers who reported less than $20 \%$ of birefringent regions of the total sponge phase area. Oily streaks as wavy sets of lines, sometimes interspersed with focal conical domains, are the most common structural defects in the lamellar liquid crystal [125]. Indeed such defects were observed in the $\mathrm{D}^{4}$ texture of our system (see Fig. 33). Morphological features in the form of striations forming a crosshatch [126,127] are attributed to the columnar rectangular phase, $\mathrm{A}^{8}$. A typical fan-shaped texture resulting from the thermomechanical undulation of the columns $[128,129]$ was observed from the columnar hexagonal phase, $\mathrm{E}^{10}$. The mesostructures at the other compositions indicated in the phase diagram (see Fig. 33) and in Table 5 exhibit similar textural features as discussed above. The fact that addition of phenyl alcohol at 5-10 mM concentration does not change the textural features proves that such small amphiphiles do not disrupt the arrangement of the SDS mesophases.

\subsubsection{Calorimetric Measurements}

The DSC profiles of the ternary mixture show that the micellar phase $\mathrm{L}_{1}{ }^{1}$ is stable at least over the temperature range from $-20{ }^{\circ} \mathrm{C}$ to $+80{ }^{\circ} \mathrm{C}$, and the lamellar phase $\mathrm{D}^{4}$ from $-20{ }^{\circ} \mathrm{C}$ up to about $+50{ }^{\circ} \mathrm{C}$.

The microemulsion $\mu \mathrm{E}^{2,3}$ and sponge phase $\mathrm{L}_{3}{ }^{6,7}$ of the quaternary mixtures are stable from $0{ }^{\circ} \mathrm{C}$ up to $80{ }^{\circ} \mathrm{C}$. In the case of the lamellar phase $\mathrm{D}^{5}$ no changes in DSC thermograms were detected between 5 and $50^{\circ} \mathrm{C}$. The rectangular $\mathrm{A}^{8,9}$ as well as the hexagonal $\mathrm{E}^{10}$ and $\mathrm{F}^{11}$ mesophase (both of ternary and quaternary mixtures), do not undergo any phase transition between 20 and $80{ }^{\circ} \mathrm{C}$, and 15 and $60{ }^{\circ} \mathrm{C}$, respectively. Probably, the addition of dodecane (freezing point $-12{ }^{\circ} \mathrm{C}$ ) changes the freezing point of the entire mixture to $-8{ }^{\circ} \mathrm{C}$ or even -10 ${ }^{\circ} \mathrm{C}$ depending on the SDS based phase, and therefore the stability of the quaternary systems is narrower.

\subsubsection{Partitioning of Phenyl Alcohols}

As it described in Subsection 4.1.2.3 the determination of the \%aqueous character, is given as a fractional shift of the resonance between the resonance fields in water $(100 \%$ aqueous character) and $n$-dodecane ( $0 \%$ aqueous character) for the SDS system: 


$$
\% \text { aq.character }=\frac{B_{S D S}-B_{\text {Dodecane }}}{B_{\text {water }}-B_{\text {Dodecane }}} \times 100
$$

where $B_{\mathrm{SDS}}, B_{\text {water }}$, and $B_{\text {Dodecane }}$ are the resonance positions for the spin probe in the SDS dispersions, in plain water and $n$-dodecane (used for calibration), respectively.

Typical ALC spectra of 3-phenylpropan-1-ol solubilized in several SDS mesophases at 25 ${ }^{\circ} \mathrm{C}$ are presented in Figure 34 and arranged in order of decreasing resonance fields of the $\Delta_{0}$ lines of the three radical isomers.

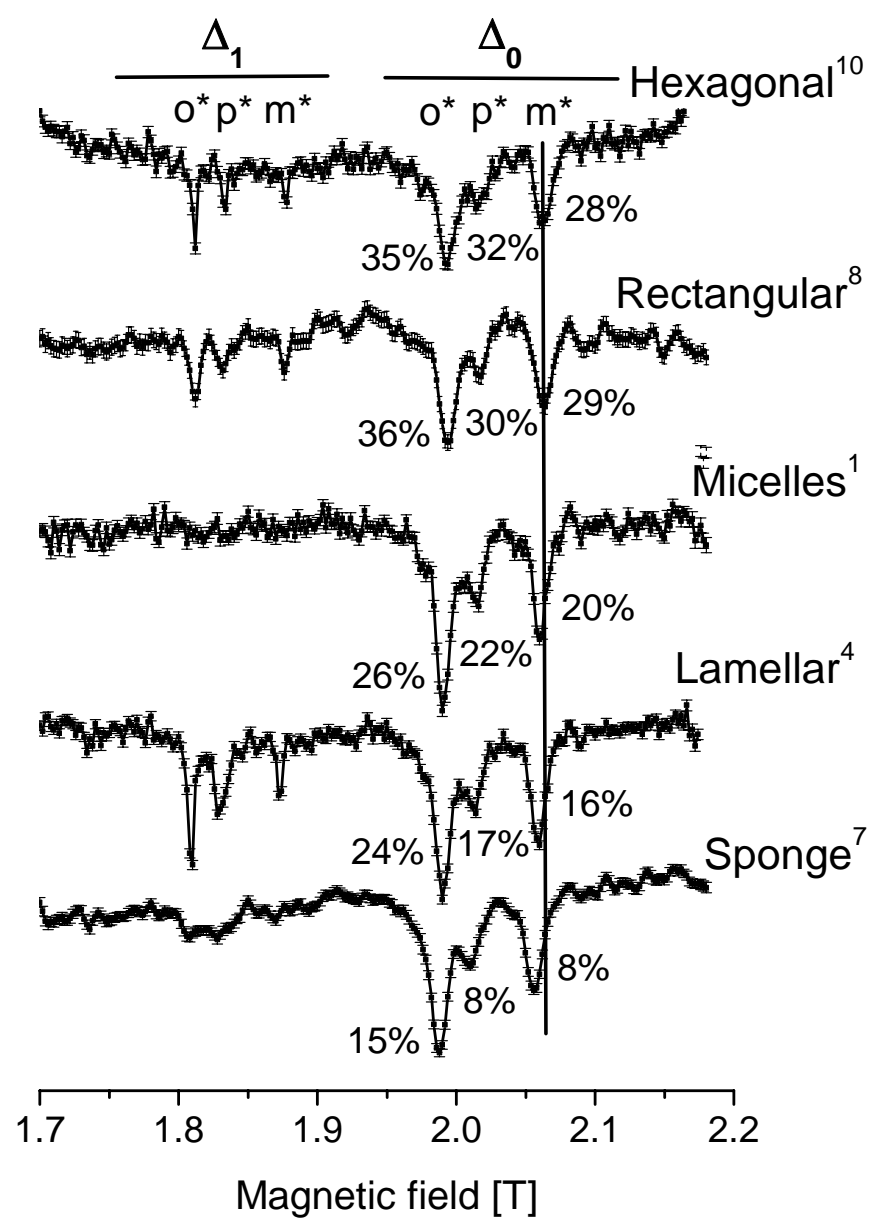

Figure 34: ALC- $\mu \mathrm{SR}$ spectra at $25{ }^{\circ} \mathrm{C}$ of $10 \mathrm{mM}$ 3-phenylpropan-1-ol in various SDS mesophases with the corresponding aqueous character given in $\%$. $\mathrm{o}^{*}, \mathrm{p}^{*}, \mathrm{~m}^{*}$ refer to the radical isomers (ortho, para, meta) incorporated within the surfactant membrane. The superscript numbers relate to the sample composition. The vertical line indicates constant aqueous character at $28 \%$.

They translate into aqueous character below $40 \%$, which means that the radical is incorporated within the SDS membrane. 
The presence of the $\Delta_{1}$ resonances in the top spectrum for the hexagonal phase $\mathrm{E}^{10}$ confirm that the probe molecule is located in the SDS columns. Depending on the radical isomer its line width ranges between only 0.8 and $1.4 \mathrm{mT}$, which by comparison with the static widths of ca. $35 \mathrm{mT}$ [23] indicates considerable dynamical averaging of the hyperfine anisotropy (for details see Section 4.2.5).

The rectangular phase $\mathrm{A}^{8}$ reveals similar ALC features as the hexagonal phase, with the exception that the line width of the $\Delta_{1}$ peaks is larger and amounts to $1.6-2.2 \mathrm{mT}$, indicating slightly different reorientational dynamics of the radical.

The absence of the $\Delta_{1}$ lines is characteristic for the micellar $\mathrm{L}_{1}{ }^{1}$ state, revealing that the radical resides in an isotropic environment, but the low aqueous character (20-26\% aq.) demonstrates that the spin probe is incorporated in the micelle rather than in water. This means that the probe reorients isotropically in the 1-pentanol, which is the dominant component in this mesophase, alternatively it may indicate that the hyperfine anisotropy averages by fast rotation of the entire micelle. Scheuermann et al. [84] using the same method to monitor the partitioning of 2-phenylethan-1-ol in micelles of the nonionic surfactant, tetra(ethyleneglycol) $n$-dodecyl ether $\left(\mathrm{C}_{12} \mathrm{E}_{4}\right)$ discussed a similar phenomenon, as in the region of the expected $\Delta_{1}$ resonances a very broad feature was hardly discriminated from the background. By comparison with Monte Carlo simulations [91] they found that the averaging occurs by rotational diffusion of the micelle on a timescale of $5 \mathrm{~ns}$.

Significantly higher amplitudes and broader $\Delta_{1}$ lines $(1.9-4.7 \mathrm{mT})$ in the lamellar phase $\mathrm{D}^{4}$ suggest a further increase of anisotropy. It is interesting to note that they are narrower for the meta and ortho than for the para isomer. The low polarity in the range of $16-24 \%$ reveals a deeper immersion of the alcohol phenyl ring into the lipid bilayer than in the other mesophases.

In spite of a large amount of dodecane, the $\Delta_{1}$ lines are also observed in the sponge phase $\mathrm{L}_{3}{ }^{7}$, although they are much broader here than in the other phases. From the aqueous character we know that the phenyl alcohol is in the SDS membrane (8-15\% aq.), located even deeper than in the lamellar mesophase. An unchanged width of the $\Delta_{0}$ lines excludes a possible interpretation of very slow rotation or even immobilization of the phenyl alcohol within the curved bilayer. As mentioned above the bilayer of the SDS sponge phase is very flexible.

The guest molecules in such a bilayer can rotate, rock, undergo flip-flop inversion, diffuse laterally or transversely, or they may even be involved in interbilayer migration. The lateral diffusion along the interface is rapid, with a diffusion constant on the order of $10^{-10}-10^{-12} \mathrm{~m}^{2}$ 
$\mathrm{s}^{-1}[5,130]$. This translates into a root mean square displacement of 3-30 nm over the muon lifetime of $2.2 \mu \mathrm{s}$. However transverse diffusion from one surface of the bilayer to the other is extremely slow, ranging from 2 to $24 \mathrm{~h}[130,131]$. $\Delta_{1}$ resonances are averaged out fully by isotropic motion on a critical time scale given by the inverse of the hyperfine anisotropy (typically $50 \mathrm{~ns}$ for cyclohexadienyl type radicals), therefore the transverse diffusion and the fluctuating curvature of the sponge phase, with undulations on a typical time scale of $10^{-6}$ $10^{-1} \mathrm{~s}$ [132], are too slow to significantly affect the signal over the muon lifetime. Thus, only diffusion along the highly curved interface can be a reasonable explanation for the broad ALC signature detected for the sponge phase.

Figure 35 illustrates a similar behaviour of the second probe molecule, 5-phenylpentan-1ol, in all SDS phases.

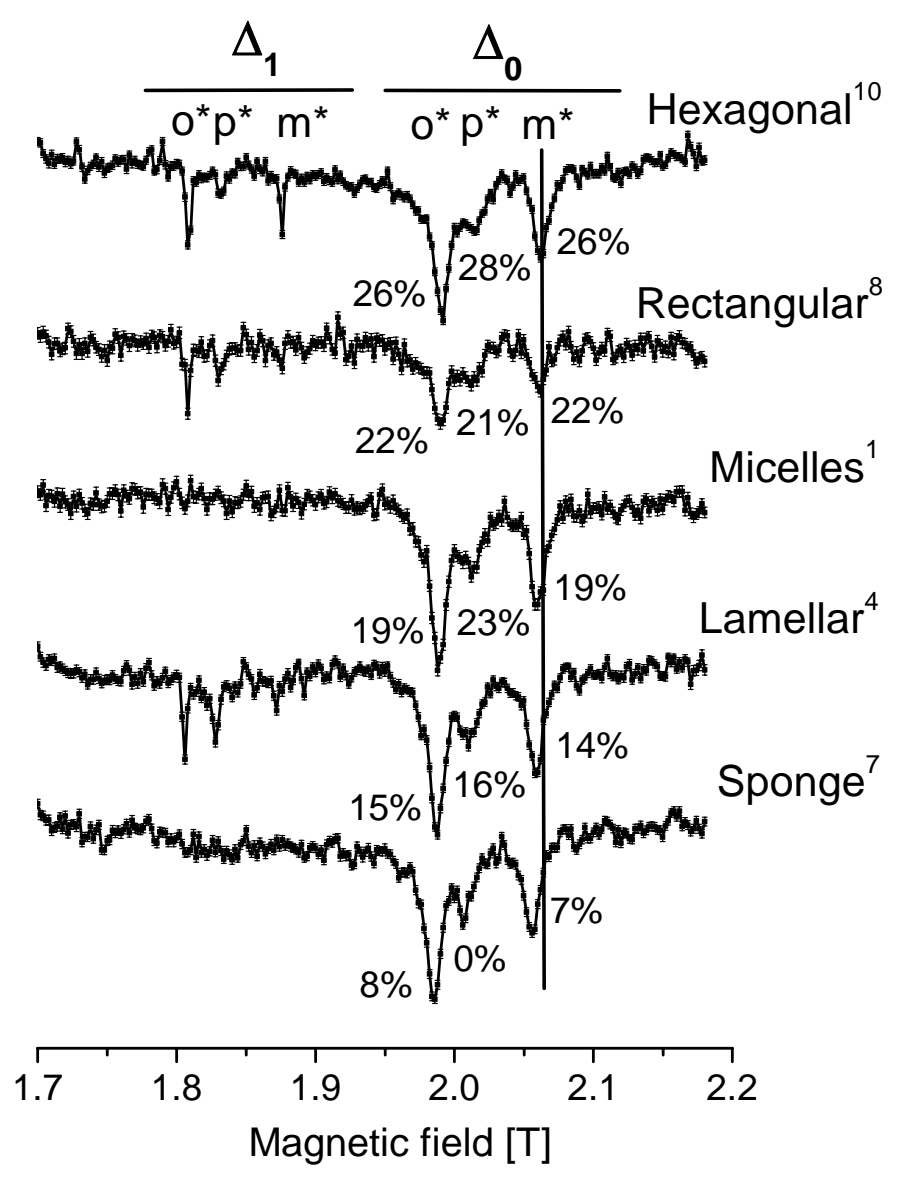

Figure 35: ALC- $\mu \mathrm{SR}$ spectra at $25{ }^{\circ} \mathrm{C}$ of $5 \mathrm{mM}$ 5-phenylpentan-1-ol in various SDS mesophases with corresponding aqueous character given in $\% . \mathrm{o}^{*}, \mathrm{p}^{*}, \mathrm{~m}^{*}$ refer to the radical isomers (ortho, para, meta) incorporated within the surfactant membrane. The superscript numbers relate to the sample composition (see Figure 33). 
It behaves as 3-phenylpropan-1-ol, although with two exceptions. Firstly, the measured polarity is lower, as may be expected due to the longer alkyl chain and therefore higher hydrophobicity of the species. Secondly, the $\Delta_{1}$ resonances vanish completely in the sponge phase, and the aqueous character of the para isomer reaches even $0 \%$, which is observed for the first time in a surfactant dispersion. Obviously, the more hydrophobic 5-phenylpentan-1ol molecule diffuses into the SDS bilayer towards the hydrocarbon core filled by dodecane, undergoing isotropic reorientational motions. Noteworthy is the fact that partitioning of both radicals into the hexagonal phase reveals an aqueous character which is the highest among all SDS mesophases (see Fig. 34, 35), which is probably due to the surfactant packing [7].

It is interesting to compare the present results of radical partitioning into the lamellar SDS phase [132] with results for DHTAC [82]. In the lamellar DHTAC dispersions both phenyl alcohols were shown to coexist between the aqueous and nonaqueous environments at $25{ }^{\circ} \mathrm{C}$, while in SDS systems at the same temperature they are located exclusively in the surfactant bilayer (no split $\Delta_{0}$ lines were observed in the case of SDS, in contrast to DHTAC [83]). This may be due to the more labile and thus more accessible membrane of the anionic surfactant, while the DHTAC alkyl chains form a rigid liquid gel-like structure at this temperature. Only above a phase transition at ca. $50{ }^{\circ} \mathrm{C}$ in which the hydrocarbon side chains of DHTAC change their packing to a fluid-like structure were the phenylalcohols incorporated completely in the bilayer. The high accessibility of the SDS membrane suggests that its hydrocarbon chains are always in a fluid-like state. This may first of all have to do with the shorter chains of SDS, secondly with the addition of 1-pentanol, which not only adds flexibility to the surfactant structure but also lowers any potential phase transition temperatures. In biological cell membranes consisting of phospholipids, which have a very similar structure to that of DHTAC, addition of cholesterol and other components serves the same purpose. It is essential for living systems that the cell membranes remain in a flexible fluid-like state so that they can accommodate functional components and even drug molecules.

Moreover, the environment polarity near the head group of DHTAC is higher than in SDS, a fact that is attributed to the polarity of the ethoxy part of the cationic surfactant and to a water gradient that extends into the bilayer [83]. Finally, in SDS the para isomer exhibits a polarity that is similar to that of the other isomers, which is not so for the DHTAC dispersion. 


\subsubsection{Effect of Dodecane Content}

Figure 36 displays ALC spectra of 3-phenylpropan-1-ol distributed in the SDS micellar phase as a function of dodecane dilution.

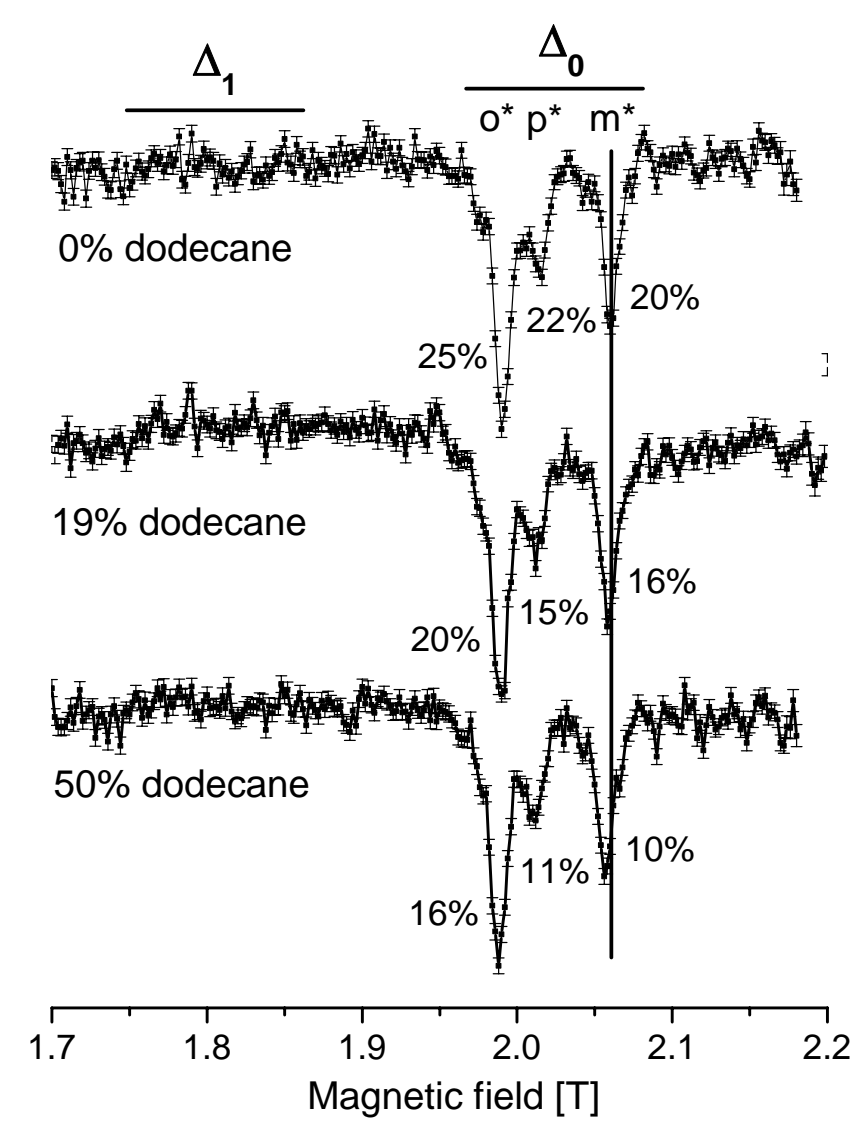

Figure 36: ALC- $\mu$ SR spectra of $10 \mathrm{mM}$ 3-phenylpropan-1-ol in the SDS micelles as function of dodecane content at $25^{\circ} \mathrm{C}$ (compositions corresponding to points 1, 2, and 3 in Figure 33).

It is intriguing to note that with increasing 'oil' fraction, the $\Delta_{0}$ resonances shift to lower fields, which is associated with decreasing aqueous character. The same phenomenon is observed for 5-phenylpentan-1-ol, revealing an even lower polarity than for the shorter alcohol, as expected due to its higher hydrophobocity. Also for the other four mesophases the addition of a certain amount of dodecane causes a shift of the $\Delta_{0}$ lines to lower field values. Simultaneously, the intensities of the $\Delta_{1}$ resonances always decrease with the fraction of dodecane due to the reduction of the anisotropy.

Thus, the addition of dodecane lowers the aqueous character that is sensed by the spin probe [133]. Based on the picture that the probe molecule is anchored at the interface this is at 
first unexpected. There are two possible explanations for this effect. One is that the addition of dodecane releases the probe molecule from its anchoring at the interface so that it can dip deeper into the micelles, but the more likely is that the reduced water content (only $6 \%$ for point 3 of the phase diagram, Figure 33) does not permit as much water to partition into the micelle as most of it is bound to the surface where it has to solvate the SDS ions.

\subsubsection{Reorientational Dynamics of $\mathrm{MuC}_{6} \mathrm{H}_{5}\left(\mathrm{CH}_{2}\right)_{3} \mathrm{OH}$}

As it has been already demonstrated in Subsection 4.1 .5 polarized positive muons substituted for hydrogen nuclei in muoniated radicals derived from the 3-phenylpropan-1-ol were used as spin labels to sense reorientational motion of the radical in the environment of the DHTAC surfactant system. The characteristic shape and width of the $\Delta_{1}$ transition allows the determination of the parallel hyperfine coupling component of the $\mathrm{Mu}$ adduct to 3phenylpropan-1-ol also in different mesophases of SDS anionic surfactant at $25{ }^{\circ} \mathrm{C}$. For restricted radical dynamics due to the limited space between the SDS amphiphiles and the near-linear geometry of the considered solute, we assume again that $\mathrm{MuC}_{6} \mathrm{H}_{5}\left(\mathrm{CH}_{2}\right)_{3} \mathrm{OH}$ can undergo only uniaxial rotation about the first $\mathrm{C}-\mathrm{C}$ substituent bond at the phenyl ring.

The calculation of the theoretical dipolar muon hyperfine coupling component parallel to this rotation axis, $D_{\|}^{\mu \text { theo }}$, was performed in the same manner as presented in $[83,118]$. Experimental values of the parallel component $\left(D_{\|}^{\mu, e x p}\right)$ of the effective uniaxial hyperfine anisotropy were evaluated by fitting the $\Delta_{1}$ signals, which are of Lorenzian shape. Inspection of table 10 clearly shows that the experimental values of $D_{\|}^{\mu, e x p}$ of the ortho and para isomers in all SDS mesophases deviate significantly from the theoretical $D_{\|}^{\mu \text { theo }}$.

Table 10: Experimental values of the parallel uniaxial hyperfine coupling component $\left(D_{\|}^{\mu, e x p}\right)$ of the $\mathrm{Mu}$ adduct to 3-phenylpropan-1-ol distributed in different SDS mixtures at $25{ }^{\circ} \mathrm{C}$. The calculated values $\left(D_{\|}^{\mu, t h e o}\right.$ ) for are for pure uniaxial rotation about the first substituent bond.

\begin{tabular}{cccccc}
\hline Isomers & $D_{\|}^{\mu, \text { theo }} / \mathrm{MHz}$ & $\begin{array}{c}D_{\|}^{\mu, \text {exp}} / \mathrm{MHz} \\
\text { Lamellar }^{4}\end{array}$ & $\begin{array}{c}D_{\|}^{\mu, \text { exp }} / \mathrm{MHz} \\
\text { Reverse lamellar }^{5}\end{array}$ & $\begin{array}{c}D_{\|}^{\mu, \text {exp}} / \mathrm{MHz} \\
\text { Rectangular }^{8}\end{array}$ & $\begin{array}{c}D_{\|}^{\mu, \text { exp }} / \mathrm{MHz} \\
\text { Hexagonal }^{10}\end{array}$ \\
\hline ortho & 3.9 & $0.4(1)$ & $0.3(1)$ & $0.3(1)$ & $0.2(1)$ \\
para & 7.1 & $1.3(4)$ & $0.9(2)$ & $0.5(1)$ & $0.3(1)$ \\
meta & 0.06 & $0.3(1)$ & $0.2(1)$ & $0.3(1)$ & $0.2(1)$ \\
\hline
\end{tabular}


This can be explained by more extensive averaging of the anisotropy than was assumed for uniaxial rotation. Moreover, the ratio between $D_{\|}^{\mu, e x p}$ (para) and $D_{\|}^{\mu, e x p}$ (ortho) amounts roughly to a factor of three for the lamellar phase, decreasing to about half of this in the case of the rectangular and hexagonal mesophases, which is close to the theoretical predictions.

The absolute effective $D_{\|}^{\mu, e x p}$ values of the ortho resonances in the lamellar phase are smaller by a factor of ten than the corresponding $D_{\|}^{\mu \text { theo }}$ values. Note that in the hexagonal phase it amounts to a value of about twenty. The same tendency is observed for the para isomer in the lamellar phase, where $D_{\|}^{\mu, e x p}$ differs from $D_{\|}^{\mu, t h e o}$ by a factor of roughly five, increasing even to twentyfour in the hexagonal mesophase. The above facts suggest that the rotation axis itself undergoes fast and extensive further motion, such as wobbling or tumbling. Moreover, the decreasing tendency of the $D_{\|}^{\mu, e x p}$ values from the lamellar towards the hexagonal phase (see Table 10) indicates more extensive reorientational dynamics of the 3phenylpropan-1-ol incorporated in the hexagonal phase than in the other SDS mesostructures. In contrast to the ortho and para species, the $D_{\|}^{\mu, e x p}$ of the meta radicals in all cases deviate from the $D_{\|}^{\mu, \text { theo }}$ only slightly (Table 10). It means very small reduction of the muon hyperfine anisotropy.

We would like to stress that our previous results of $D_{\|}^{\mu, e x p}$ obtained for 3-phenylpropan-1ol located in the DHTAC lamellar phase [83] are in agreement with those for ternary SDS mixture in the same phase [133]. However, in the quaternary system of the SDS lamellar mesostructure (reverse lamellar $\mathrm{D}^{5}$ ) the experimental values of the parallel component are slightly smaller that in the case of the cationic surfactant. Obviously, the additional fraction of dodecane (isotropic environment) permits a more free rotation of the radical [133].

\subsubsection{Conclusions}

The main goal of this part of the thesis was to show that ALC- $\mu$ SR is suitable to monitor the location and the dynamics of the muoniated 3-phenylpropan-1-ol and 5-phenylpentan-1-ol molecules in more complex environments as microemulsions, micelles, lamellar, rectangular, hexagonal and sponge phase of the SDS/water/pentan-1-ol ternary mixtures and the SDS/water/pentan-1-ol/dodecane quaternary systems. 
The present results show a dependence of the phenyl alcohol partitioning on the environment polarity and on the structure of the SDS dispersions.

Both radicals tend to be incorporated always within the mesophases at $25{ }^{\circ} \mathrm{C}$, although at different depth. The low polarity sensed by the tracer molecule indicates that among all mesophases these species are immersed the deepest in the sponge phase. Alternatively, the effect could be explained by a dependence of the water gradient in the surfactant layer on the overall water versus hydrocarbon content of a system. Additionally, the calculations of polarity of muoniated 5-phenylpentan-1-ol radical in the sponge phase revealed for the first time $0 \%$ aqueous character.

The polarity of the para isomer is comparable to the polarity of the meta and ortho isomers of both alcohols.

The fractions of pentanol and dodecane are additional factors that affect partitioning.

The changing amplitudes, widths and shapes of the $\Delta_{1}$ resonances reveal a different extent of dynamics of three isomers of the muoniated 3-phenylpropan-1-ol radical in particular mesophases. The most extensive dynamics occurs in the hexagonal phase.

Hence, it can be concluded that probe molecule partitioning depends not only on the chain length, temperature, concentration, chemical nature and on the structure of the phenyl alcohol as it was shown in Section 4.1, but also on the type of the structure of particular surfactant mesophases and their flexibility. 


\section{Chapter 5}

\section{SUMMARY}

For the first time, ALC- $\mu$ SR is shown to be a highly sensitive experimental technique for the investigation of the partitioning of probe molecules containing an aromatic ring in diverse soft matter structures. More specifically, the probes of environment were fragrance components derived from a series of phenyl alcohols incorporated in emulsions and microemulsions of the cationic and anionic surfactants in order to elucidate the main factors that contribute to cosurfactant ordering at interfaces.

The ALC- $\mu$ SR technique takes advantage of the positive muon combining with an electron, to a hydrogen-like atom that is called muonium. This atom attaches to a phenyl group, forming a cyclohexadienyl-type radical that contains the muon as a polarized spin label, providing an excellent probe even for very low phenyl alcohol concentrations. In addition, the high polarisation and the single particle counting technique make the muon an exceptionally sensitive probe. Thus, fully polarised positive muons substituted for protons in organic free radicals can be used as spin labels that reveal information about the structure, partitioning and kinetics of these radicals.

The high sensitivity of ALC- $\mu$ SR rests mainly on two effects: (i) The hyperfine coupling constants of the radicals derived by addition of the $\mathrm{Mu}$ atom to the phenyl ring are sensitive to the polarity of the environment. This effect is analogous to the one observed with nitroxide spin probes. However, $\mathrm{Mu}$ addition to the phenyl ring leads to much less change in steric requirements than insertion of nitroxide radicals so that $\mathrm{Mu}$ is considered a far less invasive probe. (ii) Even very small hyperfine anisotropies on the order of $1 \%$ of their full static values over a critical timescale of typically 50 ns lead to the presence of the $\Delta_{1}$ avoided level crossing resonance so that also weak probe molecule association to an ordered phase or their incorporation in an environment with low order parameter are detectable.

Furthermore, it is demonstrated that probe molecule concentrations in the low millimolar range are detectable. It has been proven difficult to probe such low concentrations by means of routine techniques such as NMR [134]. The high sensitivity of the $\mu$ SR techniques rests on 
the near $100 \%$ spin polarisation of the muon spin label, a level that has not been reached in conventional magnetic resonance techniques, and on the detection mode that takes advantage of a single particle counting technique rather than on induction in pick-up coils or energy absorption in resonators. It should be noted that the high muon polarisation requires neither low temperature nor high magnetic fields (permitting effects to be analysed which would be quenched in applied fields) nor are any RF pulse sequences needed to create and manipulate spin coherence.

The focus of this work is on the systematics of phenyl alcohol partitioning and reorientational dynamics in different colloidal dispersions as a function of chain length, or more specifically on the variation of probe molecule hydrophobicity.

The main results, which are mostly in accord with known concepts, are as follows:

\section{DHTAC Surfactant Dispersions}

- Partitioning of the guest molecules into the bilayer is facilitated in the high temperature $\mathrm{L}_{\alpha}$ phase where the surfactant alkane chains are disordered (this is the natural state of biomembranes) but partly suppressed in the low temperature $L_{\beta}$ phase where the chains are ordered. Chemists take advantage of the general principle of rejection of guests from a crystalline state in recrystallisation procedures for purification.

- One of the remarkable features of the ALC- $\mu S R$ technique is that it provides clear evidence of $L_{\alpha} / L_{\beta}$ phase coexistence over some extended temperature range, as in Figure 23. The distinction to coexistence of the probe molecules in the aqueous and nonaqueous environments of the $L_{\beta}$ phase, as in Figure 26, is possible by consultation of temperatures some $20^{\circ} \mathrm{C}$ below the nominal phase transition temperature.

- The aqueous character that is sensed by the cyclohexadienyl radical shifts clearly with the length $n$ of the substituent chain. For the long chains this shift is larger than in bulk ethylacetate that can be regarded to model the head group ester region. This reveals a water gradient that extends into the surfactant bilayer.

- Furtheremore, the aqueous character sensed by the para isomer of $2 \leq n \leq 4$ phenyl alcohols is very different in comparison to the ortho and meta species. Nearly the same polarity for all three isomers was observed for $n=5$.

- The concentration of the used phenyl alcohols is an additional factor, which influences the preferred location of cosurfactants in the DHTAC dispersions. However, in 
comparison with aliphatic alcohols, the bilayer-water partitioning of phenyl alcohols do not follow Traube’s rule.

- The results of Heisenberg spin exchange with $\mathrm{Ni}^{2+}$ cations provide independent confirmation that the higher phenyl alcohols $n>3$ reside mostly within the surfactant bilayer in the high temperature phase and mostly in water in the low temperature phase. Moreover, the experiment reveals that the shortest species $n=2$ behaves similar to the other alcohols in the $L_{\alpha}$ phase. However, in the $L_{\beta}$ phase 2-phenylethan-1-ol exchanges rapidly between the DHTAC/water environments.

- The bilayer/water partitioning of the present series of phenyl alcohols reflects a combination of the classical and nonclassical hydrophobic effects that depend on temperature, chain length and concentration. The decrease in the Gibbs free energy with increasing solute size is attributed to the lowering of the interfacial tension.

- The enthalpic contribution to the free enthalpy of transfer from one environment to the other is to a large extent compensated by an entropic contribution, which is typical for hydrophobic solvation. We believe that the contribution of the aliphatic part of the phenyl alcohol is compensated by an almost identical contribution from the aromatic part.

- In addition, the thermodynamic properties $\Delta G_{\mathrm{tr}}, \Delta H_{\mathrm{tr}}$ and $T \Delta S_{\mathrm{tr}}$ that determine the solute transfer from the water to the surfactant double layer prove that the distribution also strongly depends on shape, chemical nature and different structure of phenyl alcohols.

- The low widths and amplitudes of the $\Delta_{1}$ resonances provide clear evidence of the extent of dynamic averaging of the hyperfine anisotropy, which is larger than expected based on pure uniaxial rotation about the first C-C substituent bond. Not unexpectedly, this reveals an order parameter of the phenyl alcohol molecules, which is below unity. The motions that are responsible for this must be fast compared with the critical timescale of ca. 50 ns.

- Finally, the type of dichain surfactant used here differs from a biological phospholipid only by the absence of its phosphate group. The lamellar phase structures can therefore also be regarded as model cell membranes, and the partitioning of probe molecules simulates situations relevant to drug delivery under physiological conditions. This indicates possible future extensions of the present study from its fundamental aspects to practical applications. 


\section{SDS Surfactant Dispersions}

Here, ALC- $\mu$ SR has been applied to monitor location and reorientational dynamics of two different sets of muoniated cyclohexadienyl radicals derived from 3-phenylpropan-1-ol and 5phenylpentan-1-ol as tracer molecules in much more complex environments such as SDSwater-pentanol ternary mixtures and in SDS-water-pentanol-dodecane quaternary systems.

The main conclusions of this part are the following:

- Based on the environment polarity and the presence/absence of the ALC resonances, the obtained results showed that both phenyl alcohols are located among the SDS amphiphiles in all phases.

- The \%aqueous character sensed by the para isomer of 3-phenylpropan-1-ol is comparable with the polarity of the ortho and meta isomers.

- Furthermore, the more dodecane the deeper the phenyl group of both species dips into the lipid core of the SDS membrane.

- Finally, 3-phenylpropan-1-ol undergoes different types of reorientational motions in particular SDS mesophases due to the changes in structures and dynamics of the particular surfactant phases.

Comparison of 3-phenylpropan-1-ol and 5-phenylpentan-1-ol partitioning in the present ternary SDS lamellar phase with that of earlier work on a lamellar phase binary system consisting of $85 \%$ water and $15 \%$ of the cationic dichain surfactant DHTAC reveals clear differences, which are following:

- While the previous surfactant system exhibits coexistence with separate signals for the aqueous and the DHTAC bilayer environments the same probe molecules showed only a single environment, with $<20 \%$ aqueous character in the SDS lamellar phase.

- The second point is that for both the DHTAC and the SDS system the 5-phenylpentan-1ol senses a lower aqueous character than 3-phenylpropan-1-ol. This is consistent with the picture that the phenyl alcohols are anchored with their $\mathrm{OH}$ group at the interface to the aqueous environment and that the phenyl ring dips deeper into the surfactant structure when the phenyl ring is equipped with a longer side chain. The different aqueous characters then reflect a gradient of water that partitions into the surfactant structure. However, the same probe molecule sees a lower aqueous character in SDS than in DHTAC. This may be ascribed to the glycerol ester groups near the head group of DHTAC, which are absent in SDS. An ester group has a certain polarity by itself, 
which is sensed by the spin probe, but it may also attract more water molecules into the membrane.

- The polarity of the para isomer of 3-phenylpropan-1-ol and 5-phenylpentan-1-ol deviates much less from the ortho and meta species in SDS systems than in the DHTAC emulsions.

- The tendency of decreasing experimental $D_{\|}^{\mu, e x p}$ values from the lamellar towards the hexagonal phase indicates more extensive reorientational dynamics of 3-phenylpropan1-ol in the hexagonal structure than in the other SDS phases. Moreover, the para and ortho isomers exhibit much larger reduction of the muon hyperfine anisotropy (perhaps due to wobbling or tumbling motion) than the meta species. Finally, 3-phenylpropan-1ol undergoes the same motions in both binary DHTAC and ternary SDS lamellar phase. However, in the quaternary SDS mixture (reverse lamellar) this molecule rotates more freely, which is attributed to the additional fraction of dodecane.

- Finally, the type of the surfactant, the structure of the particular mesophases and the membrane flexibility are factors that should be taken also into account in order to elucidate precisely the probable location of the probe molecule.

The above facts prove that ALC- $\mu \mathrm{SR}$ is an excellent method to examine the partitioning, dynamics and thermodynamics of the probe molecule containing the aromatic group in diverse soft matter structures, at a microscopic level, even under extremely dilute guest molecule concentrations. Thus, the muoniated radical can be treated as a spectator of the changes in the properties of the colloidal surfactant systems.

Understanding the partitioning of such small amphiphiles in colloidal dispersions is essential for designing stable surfactant emulsions and microemulsions (being used as drug delivery systems), and thus for improving the quality of new products in cosmetics, detergency, food industry and pharmaceutics. Drug partitioning in liposome and in biomembrane - cell fluid systems is a closely related subject, which plays a prominent role in drug delivery. 


\section{ZUSAMMENFASSUNG}

Die Intention dieser Doktorarbeit ist es, die Effekte von Gastmolekülen auf die Struktur verschiedener Soft-Matter-Strukturen und somit die Stabilität des vollständigen Systems zu erforschen. Genauer gesagt haben wir uns darauf konzentriert, die Faktoren zu bestimmen, die sich auf die Verteilung von Duftstoffen in Emulsionen und Mikroemulsionen auswirken, welche verschiedene lyotrope Flüssigkristallmesophasen aus kationischem DHTAC- und anionischem SDS-Tensid enthalten.

Es ist bekannt, dass die gängigsten Duftstoffe [13-15] basierend auf einer Mischung von aliphatischen Alkoholen und/oder von Phenylalkoholen hergestellt werden. Bis vor kurzem jedoch wurden meistens für aliphatische Alkohole mit kurzen, mittellangen und langen Ketten qualitative und quantitative Untersuchungen der Verteilung des Gastmoleküls in der Membran des Tensides vorgenommen. Demzufolge klafft in der Literatur immer noch ein Lücke, was die Verteilung von aromatischen Alkoholen in kolloidalen Dispersionen oberflächenaktiver Substanzen angeht.

Deshalb, um wenigstens teilweise diese Lücke $\mathrm{zu}$ füllen, wurden in dieser Arbeit Sensormoleküle aus Muonium-substituierten Cyclohexadienyl-Radikalen basierend auf fünf unterschiedlichen Phenylalkoholen benutzt: 2-Phenylethan-1-ol $(n=2)$, 3-Phenylpropan-1-ol ( $n=3)$, 4-Phenylbutan-1-ol $(n=4)$, 5-Phenylpentan-1-ol $(n=5)$ und Para-propyl-2phenylethan-1-ol ( $n=2+3)$. Durch die Variation der Kohlenstoffkettenlänge (im Folgenden wird die Anzahl der Kohlenstoffatome in der Alkylkette mit $n$ bezeichnet) ändert sich die Hydrophobizität der Moleküle und wir erwarten, dass sich dieses auf ihre Verteilung zwischen dem wässrigen Teil und dem Kohlenstoff-Teil der Tensid-Dispersion auswirkt. Darauf konzentrieren wir uns hauptsächlich in dieser Arbeit.

Zuerst wurde die Verteilung einer Serie von Molekülen, welche einen aromatischen Ring besitzen, in unterschiedlichen Soft-Matter-Strukturen untersucht. Dies wurde mit der Avoided Level Crossing Myonen-Spin-Resonanzspektroskopie (ALC- $\mu S R$ ), welche eine hoch empfindliche Messtechnik ist, durchgeführt.

Die ALC- $\mu$ SR-Technik macht sich den Vorteil zu nutze, dass das positive Muon sich mit einem Elektron zu einem wasserstoffähnlichen Atom verbindet, welches Muonium genannt wird. Dieses Atom baut sich unter Bildung eines Cyclohexadienyl-Radikals, welches das 
Muon als einen kennzeichnenden polarisierten Spin enthält, in die Phenylgruppe ein. Dieses exzellente Sensormolekül bietet sich sogar für sehr geringe Konzentrationen von Phenylalkoholen an. Zusätzlich machen die hohe Polarisation und die Technik, einzelne Teilchen zu zählen, das Muon zu einem erwartungsgemäß empfindlichen Sensormolekül. Somit kann ein voll polarisiertes positives Muon, welches ein Proton in einem organischen freien Radikal ersetzt, benutzt werden als eine Spin-Kennzeichnung, welche Informationen über die Struktur, Verteilung und Kinetik dieses Radikals enthüllt.

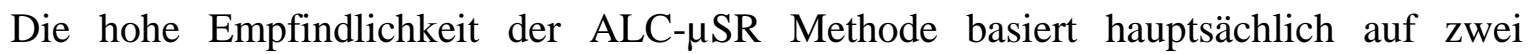
Effekten: (i) Die Hyperfeinkopplungskonstante der Radikale, welche durch die Addition eines Mu-Atoms an den Phenylring entsteht, reagiert empfindlich auf die Polarität der Umgebung. Dieser Effekt entspricht dem, den man auch bei Nitroxid-Spin-Sonden beobachtet. Doch die Addition des $\mathrm{Mu}$ an den Phenylring führt zu viel weniger Änderungen in der Sterik des Moleküls, als die Einführung des Nitroxid Radikals, so dass das Mu als ein viel weniger invasives Sensormolekül betrachtet wird. (ii) Sogar sehr kleine Hyperfein-Anisotropien in der Größenordnung von 1\% ihres ganzen statischen Wertes, über einen kritischen Zeitraum von normalerweise $50 \mathrm{~ns}$ hinweg, führen zur Anwesenheit der $\Delta_{1}$ ALC Resonanz, so dass auch schwache Verbindungen von Sensormolekülen zu einer geordneten Phase, oder ihre Einlagerung in eine Umgebung mit Parametern von geringer Ordnung nachweisbar sind. Zudem wurde bewiesen, dass auch Konzentrationen von Sensormolekülen im unteren millimolaren Bereich gemessen werden können.

Es hat sich als schwierig erwiesen, solch niedrige Konzentrationen an Hand von Routinemethoden wie z. B. NMR zu untersuchen [134]. Die hohe Empfindlichkeit der $\mu$ SRTechnik beruht auf der fast 100\%igen Spinpolarisation des kennzeichnenden Muonspins, ein Niveau, welches mit konventionellen Kernspinresonanztechniken nicht erreicht werden kann und auf dem Detektionsverfahren, welches den Vorteil hat, jedes einzelne Teilchen zu zählen, dieses ist besser als eine Induktionserfassungsspule oder ein Resonator, der die Energie absorbiert. Es ist bekannt, dass die hohe Muon-Polarisation weder niedrige Temperaturen noch hohe Magnetfelder (welche benötigt würden um gewisse Effekte zu untersuchen, die aber zu Nichte gemacht würden im angelegten Magnetfeld) benötigt. Ebenso werden keine RF Pulse gebraucht, um eine Spin-Koharenz zu schaffen und zu manipulieren.

Diese Arbeit richtet ihr Augenmerk auf die Systematik der Verteilung von Phenylalkoholen und ihre Reorientierungsdynamik in verschiedenen kolloidalen Dispersionen in Funktion der Kettenlänge oder genauer gesagt auf die Unterschiede in der Hydrophobizität 
der untersuchten Moleküle. Die wichtigsten Ergebnisse, welche meistens übereinstimmen mit den bekannten Konzepten, sind die Folgenden:

\section{DHTAC Tensid-Dispersionen}

- Die Verteilung der Gastmoleküle in der Doppelschicht ist bei hohen Temperaturen in der $\mathrm{L}_{\alpha}$ Phase, in der die Alkylketten des Tensides ungeordnet sind, erleichtert (dies ist der natürliche Zustand von Biomembranen) aber teilweise unterdrückt bei niedriger Temperatur in der $\mathrm{L}_{\beta}$-Phase, in der die Alkylketten geordnet sind. Chemiker nutzen den Vorteil des allgemeinen Prinzips der Ablehnung von Fremdstoffen im kristallinen Zustand, wenn sie um zu Reinigen umkristallisieren.

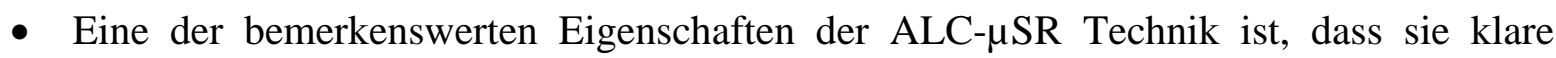
Beweise liefert für die Koexistenz der $\mathrm{L}_{\alpha} / \mathrm{L}_{\beta}$-Phase innerhalb eines ausgedehnten Temperaturbereichs, wie in Bild 23. Die Unterscheidung zwischen der Koexistenz der untersuchen Moleküle in der wässrigen und nicht-wässrigen Umgebung der $\mathrm{L}_{\beta}$-Phase, wie in Bild 26, ist möglich durch das Heranziehen von Temperaturen etwa $20^{\circ} \mathrm{C}$ unter der nominalen Phasenübergangstemperatur.

- Der Wassercharakter des Cyclohexadienyl Radikals verschiebt sich deutlich mit der Kettenlänge $n$. Für die langen Ketten ist die Verschiebung größer als in reinem Ethylacetat welches als Model für die Esterregion der Kopfgruppe herangezogen werden kann. Dies lässt einen Wassergradienten vermuten, der sich in die Doppelschicht des Tensids ausdehnt (siehe Bild 37). Ferner ist der Wassercharakter der para-Isomere des Phenylalkoholes $2 \leq n \leq 4$ sehr verschieden im Vergleich zu dem der ortho- und metaIsomere. Für alle drei Isomere mit $n=5$ wurde fast die gleiche Polarität beobachtet.

- Die Konzentration der benutzten Phenylalkohole ist ein weiterer Faktor, der Einfluss auf den bevorzugten Aufenthaltsort des Kosurfaktants in der DHTAC-Dispersion ausübt. Im Vergleich jedoch zu den aliphatischen Alkoholen folgt die Doppelschicht-Wasser Verteilung der Phenylalkohole nicht der Tauber-Regel. Die Ergebnisse von Heisenberg im Bezug auf den Spinaustausch der $\mathrm{Ni}^{2+}$-Kationen sorgt für eine unabhängige Bestätigung, dass sich die höheren Phenylalkohole $n>3$ bei hohen Temperaturen meistens innerhalb der Doppelschicht des Tensides aufhalten und in der Phase, die bei niedrigen Temperaturen existiert, meistens im Wasser. 

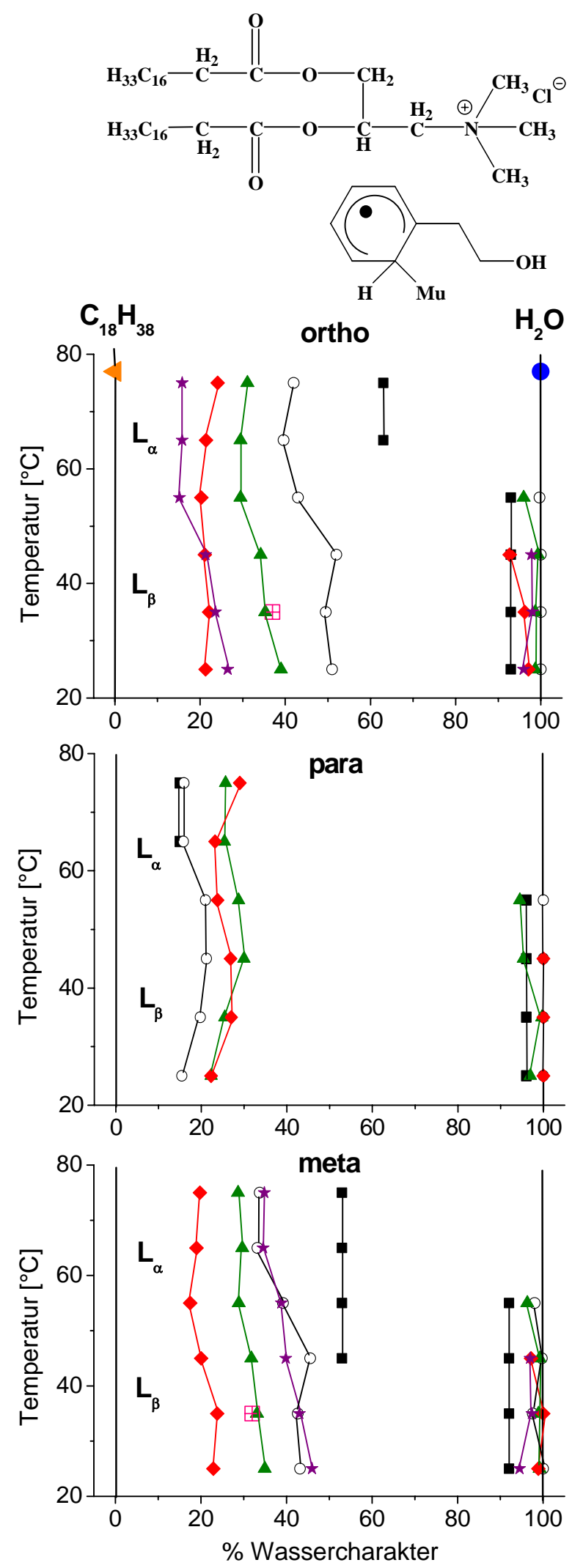

Bild 37: Struktur des doppelkettigen, kationischen Tensides DHTAC und des ortho-2Phenylethan-1-ol mit dem entsprechenden Polaritäts Diagramm für: $n=2(\boldsymbol{\square}), n=3(\bigcirc), n=$ $4(\Delta), n=5(\diamond)$ und $n=2+3(\star)$ in 15\%iger DHTAC Dispersion beziehungsweise in Wasser $(\bigcirc)$, in Ethylacetat $(\boxplus)$ und in Octadecan $(\triangleleft)$. 
- Außerdem zeigt das Experiment, dass die kürzeste Spezies mit $n=2$ sich gleich verhält wie die anderen Alkohole in der $\mathrm{L}_{\alpha}$-Phase. Indes wechselt das 2-Phenylethan-1-ol in der $\mathrm{L}_{\beta}$-Phase schnell zwischen der DHTAC und der Wasser Region.

- Die Doppelschicht/Wasser-Verteilung der untersuchten Serie von Phenylalkoholen spiegelt eine Kombination wider des klassischen und nicht-klassischen hydrophoben Effekts, der abhängig ist von der Temperatur, der Kettenlänge und der Konzentration. Die Verminderung der Gibbschen freien Energie mit dem Anstieg der Größe des gelösten Stoffs ist der Verringerung der Oberflächenspannung an der Trennungsfläche zuzuschreiben.

- Der Beitrag der Enthalpie an der freien Enthalpie des Transfers von einer Umgebung zur anderen wird zum größten Teil durch den Beitrag der Entropie ausgeglichen, welcher typisch ist für hydrophobe Lösungen. Wir glauben, dass der Beitrag des aliphatischen Teils des Phenylalkohols durch einen fast identischen Beitrag des aromatischen Teils ausgeglichen wird.

- Außerdem beweisen die thermodynamischen Eigenschaften $\Delta G_{\mathrm{tr}}, \Delta H_{\mathrm{tr}}$ und $T \Delta S_{\mathrm{tr}}$, welche bestimmend sind für den Übergang der löslichen Substanzen vom Wasser in die Doppelschicht des Tensides, dass die Verteilung auch sehr stark abhängig ist von der Form, der chemischen Natur und der unterschiedlichen Struktur der Phenylalkohole.

- Die geringe Breite und Amplitude der $\Delta_{1}$-Resonanz verschaffen uns klare Beweise über das Ausmaß der durchschnittlichen Dynamik der Hyperfein-Anisotropie, welches größer ist als erwartet, wenn man eine reine uniaxiale Rotation an der ersten substituierten C-C Bindung voraussetzt. Dies zeigt nicht unerwartet der Ordnungsparameter, des Phenylalkoholmoleküls, der kleiner Eins ist. Die Bewegungen, die für dieses verantwortlich sind, müssen verglichen werden mit dem kritischen Zeitraum von ca. 50 ns.

- Die Art der Doppelkette des Tensides, das hier benutzt wurde, unterscheidet sich von einem biologischen Phospholipid nur durch die Abwesenheit der Phosphatgruppe. Darum kann die Struktur der Lamellar-Phase auch als Model für Zellmembranen betrachtet werden, und die Verteilung der Sensormoleküle simuliert Situationen, die relevant sind für die Wirkstoffverteilung unter physiologischen Bedingungen. Dieses deutet darauf hin, dass in Zukunft eine Ausdehnung der momentanen Studie von den fundamentalen Aspekten hin zur praktischen Anwendung möglich ist. 


\section{Dispersionen mit SDS als oberflächenaktiver Substanz}

In der vorliegenden Arbeit wurde die ALC- $\mu S R$ auch verwendet, um die Lokalisierung und Umorientierung der beiden Cyclohexadienyl-Myonium-Radikale von 3-Phenylpropan-1ol und 5-Phenylpentan-1-ol als Tracer-Moleküle in viel komplexeren Umgebungen wie dem Dreistoffgemisch SDS-Wasser-Pentanol oder dem Vierstoffgemisch SDS-Wasser-PentanolDodekan zu untersuchen.

Daraus ergaben sich folgende Schlüsse:

- Ausgehend von der Polarität der Umgebung zeigt die An- oder Abwesenheit von ALCResonanzen, dass beide Phenylalkohole in allen Phasen zwischen den SDSAmphiphilen lokalisiert sind.

- Die Polartiät kann durch das para-Isomere von 3-Phenylpropan-1-ol bestimmt werden und ist mit der von ortho- und meta-Isomer vergleichbar.

- Je größer der Anteil an Dodekan, desto tiefer durchdringt die Phenylgruppe beider Alkohole den Lipidkern der SDS-Membran.

- 3-Phenylpropan-1-ol unterliegt in speziellen SDS-Mesophasen verschiedenen Reorientierungsbewegungen, die mit den Änderungen in Struktur und Dynamik der jeweiligen Phase des Tensids korrelieren.

Im Vergleich der Verteilung von 3-Phenylpropan-1-ol und 5-Phenylpentan-1-ol in dem untersuchten, lamellaren SDS-Dreistoffgemisch mit früheren Arbeiten an lamellaren Zweistoffsystemen aus 85 Prozent Wasser und 15 Prozent des kationischen, zweikettigen Tensids DHTAC zeigen sich klare Unterschiede:

- Während das frühere System eine Koexistenz in wässriger und DHTAC-DoppelschichtUmgebung zeigt, befinden sich die gleichen untersuchten Moleküle in der lamellaren SDS-Phase nur in einer einzigen Umgebung ( $<20 \%$ Polarität).

- Sowohl für DHTAC als auch für das SDS-System zeigt der 5-Phenylpentan-1-ol geringeren wässrigen Charakter als für 3-Phenylpropan-1-ol. Dies passt zu der Vorstellung, dass Phenylalkohole mit ihrer OH-Gruppe an die Phasengrenze der wässrigen Umgebung anhaften und dass der Phenylring umso tiefer in die Tensidbereiche eindringt, je länger seine Seitenkette ist. Dadurch spiegelt der unterschiedliche wässrige Charakter den Gradienten der Wasserverteilung in der Tensidstruktur wider. Dennoch sieht sich das gleiche Sondenmolekül in SDS einem geringeren Wasseranteil gegenüber als in DHTAC. Dies kann von der Glycerol-Ester- 
Gruppe nahe der Kopfgruppe des DHTACs herrühren, die in SDS fehlt. Diese birgt, wie auch die Spinsonde zeigt, per se eine gewisse Polarität in sich und kann zudem mehr Wassermoleküle an die Membran binden.

- Die Polarität der para-Isomeren von 3-Phenylpropan-1-ol und 5-Phenylpentan-1-ol unterscheidet sich von ihrer Meta- und Ortho-Spezies deutlich weniger in SDS als in DHTAC.

- Die tendenziell stärke Zunahme in den experimentellen $D_{\|}^{\mu \text { exp }}$-Werten der lamellaren gegenüber der hexagonalen Phase deutet auf eine extensivere Reorientierungsbewegung von 3-Phenylpropan-1-ol in der hexagonalen Struktur als in den anderen SDS-Phasen hin. Darüber hinaus zeigen para- und ortho-Isomere eine sehr viel größere Abnahme in der Myonium-Hyperfein-Anisotropie (eventuell aufgrund von Kipp- und Wiegebewegungen) als die Meta-Spezies. Schließlich geht 3-Phenylpropan-1-ol die gleichen Bewegungen in DHTAC-Zweistoffgemischen als in dem lamellaren SDSDreistoffsystem ein. Andererseits ist die Rotation des Molekül in der SDSVierkomponentenmischung (umgekehrte Lamellenstruktur) aufgrund des zusätzlichen Dodekans erleichtert.

- Letzten Endes muss also sowohl die Art des Tensids als auch die Struktur der jeweiligen Mesophase und die Membranflexibilität in Betracht gezogen werden, wenn es darum geht, die wahrscheinliche Lokalisierung des untersuchten Moleküls genau aufzuklären.

Die oben beschriebenen Ergebnisse und Fakten bestätigen, dass ALC- $\mu$ SR eine exzellente Methode zur Untersuchung von Verteilung, Bewegung und Thermodynamik des aromatischen Sondenmoleküls in verschiedenen, mikroskopischen Soft-Matter-Strukturen, sogar für besonders niedrige Konzentration an Gastmolekülen ist. Deshalb kann das Myonium-Radikal als ein Indikator für die Veränderungen der Eigenschaften in kolloidalen Tensidsystemen herangezogen werden.

Das Verständnis der Verteilung solcher kleiner Amphiphile in kolloidalen Dispersionen ist unabdingbar, um stabile Tensidemulsionen und Mikroemulsionen (zur Wirkstoffverteilung im Körper) herstellen und diese für ihren Einsatz als neue Produkte in der Kosmetik-, Waschmittel-, Nahrungsmittel- und pharmatzeutischen Industrie verbessern zu können. Die Verteilung von Wirkstoffen in Liposomen und in Systemen aus Biomembranen und Zellflüssigkeit ist damit eng verknüpft und spielt eine wichtige Rolle für deren Effektivität. 


\section{ABBREVIATIONS}

$\Delta_{0}$

$\Delta_{1}$

$\Delta_{2}$

$\mu \mathrm{E}$

A

AHA

ALC- $\mu S R$

$\mathrm{C}_{10} \mathrm{E}_{6}$

$\mathrm{C}_{12} \mathrm{E}_{4}$

CMC

D

DDAB

DHTAC

DMPC

DPPC

DSC

DTAB

$\mathrm{E}$

EO

F

FWHM

GS

HTAB

HWHM

$\mathrm{L}_{\mathrm{c}}$

$\mathrm{L}_{\beta}, \mathrm{L}_{\beta}$,

$\mathrm{L}_{\alpha}$
Resonance due to a muon-proton spin flip-flop

Resonance due to a muon spin flip

Resonance due to a muon-proton spin flip-flip

Microemulsion

Rectangular phase

Alpha hydroxyl acid

Avoided Level Crossing Muon Spin Resonance

Polyoxyethylene(6)decanol

Tetra(ethyleneglycol) $n$-dodecyl ether

Critical Micelle Concentration

Lamellar phase

Didodecyldimethylammonium bromide

2,3-diheptadecyl ester ethoxypropyl-1,1,1-trimethylammonium chloride

Dimyristoylphosphatidylcholine

Dipalmitoylphosphatidylcholine

Differential Scanning Calorimetry

Dodecyltrimethylammonium bromide

Hexagonal phase

Ethylene Oxide

Reverse hexagonal phase

Full Width at Half Maximum

Gemini Surfactant

Hexadecyltrimethylammonium bromide

Half Width at Half Maximum

Subgel

Gel phase

Liquid crystalline or fluid phase 
$\mathrm{L}_{1}$

$\mathrm{L}_{3}$

$\mathrm{m}$

$\mathrm{Mu}$

NaDS

NMR

o

$\mathrm{p}$

$\mathrm{P}_{\beta}$,

PO

POM

PSI

RF

SANS

SAXS

SDBS

SDS

UV-Vis

W/S

ZSM-5
Micelle

Sponge phase

meta

Muonium

Sodium Dodecyl Sulphate

Nuclear Magnetic Resonance

ortho

para

Ripple phase

Propylene Oxide

Polarised Optical Microscopy

Paul Scherrer Institute

Radio-Frequency

Small Angle Neutron Scattering

Small Angle X-Ray Scattering

Sodium dodecylbenzene

Sodium Dodecyl Sulphate

Ultraviolet-Visible spectroscopy

Water to Surfactant Ratio

Zeolite ZSM-5 with a threedimensional system 


\section{List of Figures}

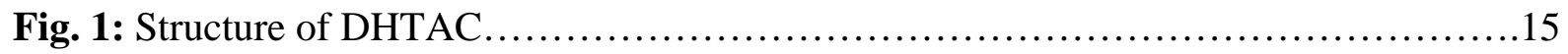

Fig. 2: Two types of Gemini surfactants. $H$ denotes ionic or nonionic headgroup. Adopted

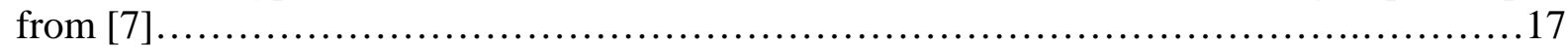

Fig. 3: Variation of surface tension with concentration for a pure aqueous surfactant solution.............................................................................

Fig. 4: Common lyotropic liquid crystalline mesophases: (a) normal micellar cubic phase ( $\left.\mathrm{I}_{1}\right)$, (b) normal hexagonal phase (E), (c) normal lamellar phase $\left(\mathrm{L}_{\alpha}\right)$, (d) inverted sponge phase $\left(\mathrm{L}_{3}\right)$, (d) normal bicontinuous cubic phase $\left(\mathrm{V}_{1}\right)$, (f) shell like structure of unilamellar vesicles, where $\delta$ denotes the thickness of the water layer and d refers to the cell size of the membrane. Figure 4c, e, f adopted from ref. [51], and Figure 4d from ref. [52]......................22

Fig. 5: Various bilayer phases. Adopted from [53]...................................23

Fig. 6: Principal radii of curvature of a saddle surface in three dimensions. Adopted from

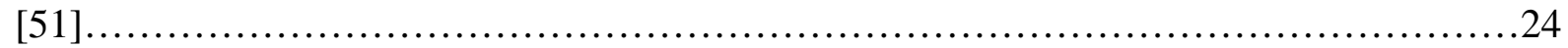

Fig. 7: The packing of surfactant molecules in a spherical micelle $\ldots \ldots \ldots \ldots \ldots \ldots \ldots \ldots \ldots . .26$

Fig. 8: Surfactant packing parameter for various aggregate structures. Adopted from ref. [57] .26

Fig. 9: Structure and chain length of phenyl alcohol guest molecules. The phenyl alcohols are distinguished by the number, $n$, of $\mathrm{CH}_{2}$ groups in their hydrocarbon chain: 2-phenylethan-1-ol $(n=2)$, 3-phenylpropan-1-ol $(n=3)$, 4-phenylbutan-1-ol $(n=4)$, 5-phenylpentan-1-ol $(n=5)$ and p-propyl-2-phenylethan-1-ol $(n=2+3)$. It is anticipated that the alcohol resides in water or in the bilayer with the HO-group at the surfactant-water interface. Probing of the environment occurs by a fully polarised muon spin label that is introduced by addition of the muonium atom to the phenyl ring which dips into the bilayer to different depths. The length of the fully stretched-out guest molecule is given in $\AA$....

Fig. 10: Formation of the muoniated cyclohehaxadienyl radical derived form benzene. Adopted from [83]....

Fig. 11: General scheme of the production of the positive muon, muonium and muoniated cyclohexadienyl radical derived from $\mathrm{C}_{6} \mathrm{H}_{5}\left(\mathrm{CH}_{2}\right)_{3} \mathrm{OH}$ in the ALC- $\mu$ SR.

Fig. 12: (a) Schematic drawing of the experimental setup for the ALC- $\mu$ SR experiment, (b) ALC- $\mu$ SR spectrum of the $\mathrm{C}_{6} \mathrm{H}_{6} \mathrm{Mu}$ radical obtained with benzene adsorbed on siliceous ZSM5 zeolite in the region of the $\Delta_{1}$ and $\Delta_{0}$ methylene proton resonances. Note that the $\Delta_{1}$ 
represents the static case in the absence of any dynamic averaging is of much higher intensity than the $\Delta_{0}$ line. Figure adopted from [83]. .36

Fig. 13: High-field energy-level diagram for a three spin system with electron $(e)$, muon $(\mu)$ and proton $(p)$. Dotted lines refer to avoided level crossings.

Fig. 14: Simulated ALC- $\mu$ SR spectra of the $\mathrm{C}_{6} \mathrm{H}_{6} \mathrm{Mu}$ radical (a) for the fully static case, (b) for fast rotation about an axis in plane through the methylene CHMu group, (c) for fast rotation about an axis perpendicular to the molecular plane, and (d) for fast rotation about an axis through an ortho carbon atom and the centre of the phenyl ring. . .40

Fig. 15: Brass sample cell for ALC- $\mu$ SR measurements . .44

Fig. 16: ALC spectrometer with 5 Tesla solenoid. .46

Fig. 17: ALC- $\mu$ SR spectrum of $18 \mathrm{mM} 3$-phenylpropan-1-ol in 15\% aq. DHTAC emulsion at $75{ }^{\circ} \mathrm{C}$ before subtraction background.

Fig. 18: Fitted ALC- $\mu$ SR spectrum with subtracted background of $18 \mathrm{mM}$ 3-phenylpropan-1ol in $15 \%$ aq. DHTAC emulsion at $75^{\circ} \mathrm{C}$. A red line is attributed to the fitted six resonances of the Lorentzian line shape. .48

Fig. 19: Solubility of phenyl alcohols in water at $25^{\circ} \mathrm{C}: n=3(\bigcirc), n=4(\Delta), n=5(\diamond)$ and $n=2+3$

Fig. 20: DSC traces showing the $\mathrm{L}_{\beta} / \mathrm{L}_{\alpha}$ phase transition of $15 \%$ aq. DHTAC without and with added phenyl alcohol at 5-40 mM concentration as a function of $n\left(\mathrm{CH}_{2}\right)$. The dashed vertical line marks the transition temperature. The dotted baseline drawn for $n=2$ demonstrates that the phase transition is broad and starts well below its nominal temperature, leading to $\mathrm{L}_{\beta} / \mathrm{L}_{\alpha}$ phase coexistence over a temperature range of nearly $20^{\circ} \mathrm{C}$

Fig. 21: Methylene $\Delta_{0}$ resonances of the three isomers of muoniated 3-phenylpropan-1-ol in water (top), in bulk liquid alcohol (middle), and in octadecane (bottom) at $75{ }^{\circ} \mathrm{C}$. The assignment of the lines to specific isomers is analogous to that of the radicals derived from 2phenylethan-1-ol [84,98]....

Fig. 22: Methylene $\Delta_{0}$ resonances of the two isomers of muoniated para-propyl-2phenylethan-1-ol in water (top), and in octadecane (bottom) at $75{ }^{\circ} \mathrm{C} \ldots \ldots \ldots \ldots \ldots \ldots \ldots \ldots . .53$

Fig. 23: ALC- $\mu$ SR spectra of $18 \mathrm{mM} 3$-phenylpropan-1-ol in 15\% aq. DHTAC emulsion. The asterisk marks the peaks relating to the radical incorporated within the bilayer. The other peaks correspond to the aqueous phase. Background correction was done by baseline subtraction.

Fig. 24: ALC- $\mu$ SR spectra of $16 \mathrm{mM}$ 4-phenyl-butan-1-ol in 15\% aq. DHTAC. The asterisk marks the alcohol located within the bilayer. Other peaks relate to water. Background correction was done by baseline subtraction. .56 
Fig. 25: ALC- $\mu$ SR spectra of $4 \mathrm{mM}$ para-propyl-2-phenylethan-1-ol in 15\% aq. DHTAC. The asterisk marks the alcohol located within the bilayer. Other peaks relate to water. Background correction was done by baseline subtraction. .57

Fig. 26: ALC- $\mu \mathrm{SR}$ spectra of $40 \mathrm{mM}$ muoniated 5-phenylpentan-1-ol in 15\% aq. DHTAC dispersion. Background correction was done by baseline subtraction. The scale bar is for the asymmetry

Fig. 27: ALC- $\mu$ SR spectra of $6 \mathrm{mM}$ muoniated 5-phenylpentan-1-ol in 15\% aq. DHTAC dispersions. Background correction was done by baseline subtraction. .59

Fig. 28: Structure of the dichain cationic surfactant DHTAC and of the ortho-2-phenylethan1-ol with corresponding polarity diagram for: $n=2(\boldsymbol{\square}), n=3(\bigcirc), n=4(\Delta), n=5(\diamond)$ and $n=2+3(\star)$ in $15 \%$ DHTAC dispersion, in water $(\mathcal{O})$, in ethyl acetate $(\boxplus)$ and in octadecane $(\triangleleft)$, respectively. .60

Fig. 29: Water gradient into the lamellar phase as determined from the meta (a) and the ortho (b) isomer of the series of phenyl alcohols. 62

Fig. 30: Methylene $\Delta_{0}$ resonances of the three isomers of the Mu adduct to 5-phenylpentan-1ol in bulk liquid alcohol and in $15 \%$ aq. DHTAC at $55^{\circ} \mathrm{C}$ (top) and at $25^{\circ} \mathrm{C}$ (bottom).......71

Fig. 31: ALC- $\mu \mathrm{SR}$ spectra of $40 \mathrm{mM}$ muoniated 2-phenylethan-1-ol in DHTAC/water dispersion with different $\mathrm{Ni}^{2+}$ concentrations at $55^{\circ} \mathrm{C}$ .74

Fig. 32: (a) Experimental $\Delta_{1}$ and $\Delta_{0}$ resonances of the Mu adduct to phenylpropanol obtained at $75{ }^{\circ} \mathrm{C}$ (squares) and simulated ALC- $\mu$ SR spectra of the three isomers assuming the radicals are rotating about the first substituent bond without any further reduction in hyperfine anisotropy (solid lines). (b) Methylene muon dipolar hyperfine tensor represented by an ellipsoid for the $\mathrm{Mu}$ adducts in the ortho, para and meta positions of the alkanol substituent. The arrow shows the component that is retained when the ring rotates about the first substituent bond. .78

Fig. 33: The partial triangular phase diagram of the quaternary SDS, water, pentanol, dodecane system at $25{ }^{\circ} \mathrm{C}$ [124], with corresponding textures of the SDS mesophases comprising phenylalcohols in polarized light microscopy. The water-to-surfactant weight ratio (W/S $=1.552 \pm 0.002)$ is kept constant. $\mu \mathrm{E}$ is the microemulsion, $\mathrm{D}$ is the lamellar phase, $\mathrm{L}_{3}$ is the sponge phase, $\mathrm{A}$ is the rectangular phase and $\mathrm{E}$ is the hexagonal phase. The numbered dots indicate the mixtures studied, and open circles stand for mixtures without dodecane while filled dots represent samples with dodecane. In the text they are given as superscripts to the phase symbol. Dashed areas refer to the coexistence of different phases. .84

Fig. 34: ALC- $\mu$ SR spectra at $25{ }^{\circ} \mathrm{C}$ of $10 \mathrm{mM}$ 3-phenylpropan-1-ol in various SDS mesophases with the corresponding aqueous character given in $\% . \mathrm{o}^{*}, \mathrm{p}^{*}, \mathrm{~m}^{*}$ refer to the radical isomers (ortho, para, meta) incorporated within the surfactant membrane. The superscript numbers relate to the sample composition. The vertical line indicates constant aqueous character at $28 \%$. . .86 
Fig. 35: ALC- $\mu$ SR spectra at $25^{\circ} \mathrm{C}$ of $5 \mathrm{mM}$ 5-phenylpentan-1-ol in various SDS mesophases with corresponding aqueous character given in \%. $\mathrm{o}^{*}, \mathrm{p}^{*}, \mathrm{~m}^{*}$ refer to the radical isomers (ortho, para, meta) incorporated within the surfactant membrane. The superscript numbers relate to the sample composition.......................................................

Fig. 36: ALC- $\mu$ SR spectra of $10 \mathrm{mM}$ 3-phenylpropan-1-ol in the SDS micelles as function of dodecane content at $25{ }^{\circ} \mathrm{C}$ (compositions corresponding to points 1, 2, and 3 in Figure 33)...90

Bild 37: Struktur des doppelkettigen, kationischen Tensides DHTAC und des ortho-2Phenylethan-1-ol mit dem entsprechenden Polaritäts Diagramm für: $n=2(\boldsymbol{\square}), n=3(\bigcirc), n=$ $4(\Delta), n=5(\diamond)$ und $n=2+3(\star)$ in 15\%iger DHTAC Dispersion beziehungsweise in

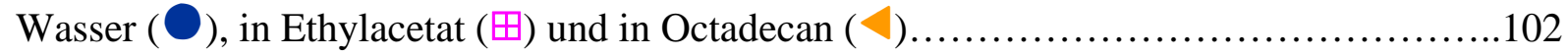




\section{List of Tables}

Table 1: Anionic surfactants...................................................... 12

Table 2: Cationic surfactants....................................................14

Table 3: Non-ionic surfactants. (EO) denotes ethylene oxide and (PO) propylene oxide to give EO/PO copolymers. The number of moles of ethylene oxide per mole of lipophile is

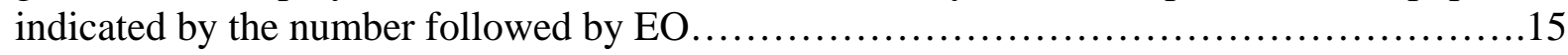

Table 4: Amphoteric surfactant.......................................................

Table 5: Compositions of the ternary and quaternary SDS samples with a constant weight

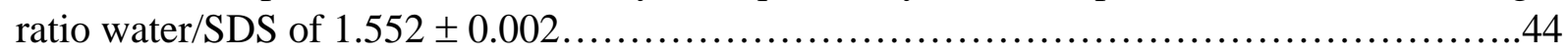

Table 6: Gibbs free energy change $\left(\Delta G_{\mathrm{tr}}\right.$ in $\left.\mathrm{kJ} \mathrm{mol}^{-1}\right)$ for the transfer of the phenyl alcohols from the water into the bilayer at their $25{ }^{\circ} \mathrm{C}$ solubility limit as measured from the $\Delta_{0}$

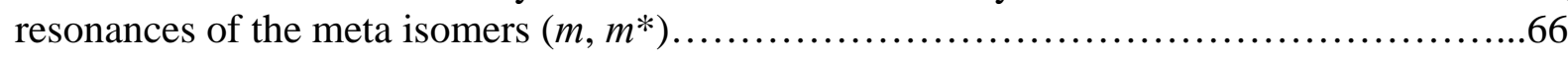

Table 7: Experimental values of enthalpy and entropy for phenyl alcohol transfer from the water into the bilayer at their $25{ }^{\circ} \mathrm{C}$ solubility limit in the temperature range from 25 to 55 ${ }^{\circ} \mathrm{C}$

Table 8: Spin exchange rate constants for the meta isomer of the $n=2$ radical in water and in $15 \%$ aq. DHTAC solution.

Table 9: Parallel uniaxial hyperfine coupling components of the $\mathrm{Mu}$ adduct to 3phenylpropanol: experimental ( $D_{\|}^{\mu, e x p}$ ) and calculated values for pure uniaxial rotation about the first substituent bond ( $D_{\|}^{\mu \text { theo }}$ and $D_{\|}^{p \text { theo }}$, see text).

Table 10: Experimental values of the parallel uniaxial hyperfine coupling component $\left(D_{\|}^{\mu, e x p}\right)$ of the Mu adduct to 3-phenylpropanol distributed in different SDS mixtures at $25{ }^{\circ} \mathrm{C}$. The calculated values $\left(D_{\|}^{\mu, t h e o}\right.$ ) for are for pure uniaxial rotation about the first substituent bond..91 


\section{BIBLIOGRAPHY}

[1] R.A.L. Jones, Soft Condensed Matter, University Press, Oxford (2002).

[2] A.T. Skjeltorpand and A.V. Belushkin, Forces, Growth and Form in Soft Condensed Matter: At the Interface Between Physics and Biology, Kluwer Academic Publisher (2004).

[3] P-G. de Gennes, Soft Matter, 1, 16 (2005).

[4] I.W. Hamley, Introduction to Soft Matter, John Wiley \& Sons, New York (2000).

[5] D.B. Goldstein, Ann. Rev. Pharmacol. Toxicol., 24, 43 (1984).

[6] E. Roduner, Physica B, 326, 19 (2003).

[7] T. Hargreaves, Chemical Formulation, An Overview of Surfactant-Based Preparations Used in Everyday Life, The Royal Society of Chemistry, Cambridge (2003).

[8] H. Stegemeyer, Liquid Crystals, Steinkopff, Darmstadt, Springer, New York (1994).

[9] P. Kraft, K.A.D. Swift, Perspectives in Flavor and Fragrance Research, VHCA, Zurich (2005).

[10] K. Bauer, D. Garbe, H. Surburg, Common Fragrance and Flavor Materials, WileyVCH, Weinheim (2001).

[11] L.L. Schramm, Surfactants: Fundamentals and Applications in the Petroleum Industry, University Press, Cambridge (2000).

[12] L.L. Schramm, Emulsions, Foams, and Suspensions, Wiley-VCH, Weinheim (2005).

[13] D. Pybus and Ch. Sell, The Chemistry of Fragrances, The Royal Society of Chemistry, Cambridge (1999).

[14] S.C. Smolinske, Handbook of Food, Drug, and Cosmetic Excipients, CRC Press, New York, (1992).

[15] M. Browning, 300 Handcrafted Soaps, Sterling Publishing Co, Inc. (2004).

[16] Muon Science: Muons in Physics, Chemistry and Materials, ed. S.L. Lee, S.H. Kilcoyne and R. Cywinski, Proceedings of the $51^{\text {st }}$ Scottish Universities Summer 
School in Physics, St. Andrews 1998, Scottish Universities Summer School in Physics and Institute of Physics Publishing, Bristol, pp. 481 (1999).

[17] E. Roduner, The Positive Muon as a Probe in Free Radical Chemistry, Potential and Limitations of the $\mu$ SR Technique, Lecture Notes in Chemistry, Springer, Heidelberg, 49, 104 (1988).

[18] J.W. Schneider, Avoided Level Crossing: A New Technique in Muon Spin Rotation to Study the Nuclear Hyperfine Structure of Muonium Centres in Semiconductores, Ph.D. thesis, University of Zürich (1989).

[19] D.G. Fleming, D.J. Arseneau, J.J. Pan, M.Y. Shelley, M. Senba, and P.W. Percival, Appl. Magn. Reson., 13, 181 (1997).

[20] F.L. Pratt, S.J. Blundell, Th. Jestädt, B.W. Lovett, A. Husmann, I.M. Marshall, W. Hayes, A. Monkman, I. Watanabe, K. Nagamine, R.E. Martin. A.B. Holmes, Physica $B$, 289-290, 625 (2000).

[21] B.W. Lovett, J.S. Stießberger, S.J. Blundell, Th. Jestädt, A. Ardavan, I.M. Marshall, F.L. Pratt, I.D. Reid, Physica B, 289-290, 612 (2000).

[22] B.W. Lovett, S.J. Blundell, J.S. Stießberger, F. L. Pratt, Th. Jestädt, W. Hayes, S. P. Cottrell, I. D. Reid, Physical Review B, 63, 054204 (2001).

[23] E. Roduner, M. Stolmár, and H. Dilger, J. Phys. Chem. A, 102, 7591 (1998).

[24] B. Beck, Ph.D. thesis, University of Stuttgart (2003).

[25] K.H. Chow, B. Hitti, R.F. Kiefl, in: M. Starola (Ed.), Identification of Defects in Semiconductors, Semiconductors and Semimetals, Academic Press, New York, 51A, 137 (1998).

[26] S.R. Kreitzman, J.H. Brewer, D.R. Harshman, R. Keitel, D.Ll. Williams, K.M. Crowe and E.J. Ansaldo, Phys. Rev. Letters, 56, 181 (1986).

[27] K.M. Layn, P.G. Debenedetti, and R.K. Prud'homme, J. Chem. Phys., 109, 5651 (1998).

[28] B.S. Sekhon, Resonance: Journal of Science Education, 9, 42 (2004).

[29] B.P. Binks, Modern Aspects of Emulsion Science, The Royal Society of Chemistry, Cambridge (1998).

[30] J.N. Israelachvili, Intermolecular and Surface Forces, With Applications to Colloidal Systems, Academic Press, London (1985). 
[31] D. Myers, Surface, Interface, and Colloids, John Wiley \& Sons, New York (1999).

[32] K. Tsujii, Surface Activity, Principles, Phenomena, and Applications, Academic Press, San Diego (1998).

[33] H. Mohrbach, The Journal of chemical Physics, 123, 126101 (2005).

[34] C. Tanford, Proc. Natl. Acad. Sci., 76, 4175 (1979).

[35] W. Blokzijl and J. Engbert, Angew. Chem. Int. Ed. Engl., 32, 1545 (1993).

[36] G. Graziano, Phys. Chem. Chem. Phys., 1, 3567 (1999).

[37] N. Muller, Acc. Chem. Res., 23, 23 (1990).

[38] W. Kauzmann, Adv. Protein Chem., 14, 1 (1959).

[39] E. Rowe, F. Zhang, T. Wu Leung, J.S. Parr, and P.T. Guy, Biochemistry, 37, 2430 (1998).

[40] D. Chandler, Nature, 437, 640 (2005).

[41] M.J. Rosen, Surfactants and Interfacial Phenomena, John Wiley \& Sons, New York (1988).

[42] E. Gallicchio, M.M. Kubo, and R. Levy, J. Phys. Chem. B, 104, 6271 (2000).

[43] G. Graziano, J. Chem. Soc., Faraday Trans., 4, 3345 (1998).

[44] H.S. Frank, M.W. Evans, J. Chem. Phys., 13, 507 (1945).

[45] N.T. Southall, K.A. Dill, Biophysical Chemistry, 101-102, 295 (2002).

[46] W.C. Wimley and S.H. White, Biochemistry, 32, 6307 (1993).

[47] J. Seelig and P. Ganz, Biochemistry, 30, 9354 (1991).

[48] K. Holmberg, Handbook of Applied Surface and Colloid Chemistry, John Wiley \& Sons, England (2001).

[49] P.M. Chaikin, T.C. Lubensky, Principles of Condensed Matter Physic, University Press, Cambridge (2000).

[50] K. Holmberg, B. Jönsson, B. Kronberg and B. Lindman, Surfactants and Polymers in Aqueous Solution, John Wiley \& Sons Ltd, England (2003).

[51] D.F. Evans, H. Wennerströrm, The Colloidal Domain, Where Physics, Chemistry, and Biology Meet, Wiley-VCH, New York (1999).

[52] M. Magalhaes, A.M.F. Neto, and A.C. Tromba, J. Phys. Chem. B, 108, 15962 (2004).

[53] M. Kranenburg and B. Smit, J. Phys. Chem. B, 109, 6553 (2005). 
[54] B.A. Cunningham, A.D. Brown, D.H. Wolfe, W.P. Williams, and A. Brain, Physical Review E, 58, 3662 (1998).

[55] I. Danielsson, B. Lindman, Colloids Surf. A, 3, 391 (1981).

[56] M. Davidson, Ph.D. thesis, University of Göteborg (2003).

[57] Gennis, RB. Biomembranes, Springer-Verlag, New York, (1989).

[58] K. Esumi, M. Ueno, Structure-Perfomance Relationships in Surfactant, Marcel Dekker, New York (2003).

[59] A. Graciaa, J. Lachaise, C. Cucuphat, M. Bourrel, and J.L. Salager, Langmuir, 9, 3371 (1993).

[60] G.I. Cherednichenko, L.I. Tsegel'nyuk, V.Ya. Kulik, and A.V. Fedorenko, Chemistry and Technology of Fuels and Oils, Springer, New York, 25, 317 (1989).

[61] R. Strey, M. Jonströmer, J. Phys. Chem., 96, 4537 (1992).

[62] B. Matliasson, H. Kaul, R. Hatti-Kaul, Isolation and Purification of Proteins, Marcel Dekker, New York (2003).

[63] K.S. Birdi, Handbook of Surface and Colloid Chemistry, CRC Press, New York (1997).

[64] W.M. Gelbard, W.E. McMullen, A. Masters, A. Ben-Shaul, Langmuir, 1, 101 (1985).

[65] A. Patist, T. Axelbert, and D.O. Shah, J. Coll. Inter. Sci., 208, 259 (1998).

[66] M. Rasmusson, U. Olsson, Progr. Colloid Polym. Sci., 120, 74 (2002).

[67] B. Farago, D. Richter, J.S. Huang, S.A. Safran, S.T. Milner, Phys. Rev. Lett., 65, 3348 (1990).

[68] J.M.Di. Meglio, M. Dvolaitzky, Ch. Taupin, J. Phys. Chem., 89, 871, (1985).

[69] G. Palazzo, F. Lopez, M. Giustini, G. Colafemmina, A. Ceglie, J. Phys. Chem. B, 107, 1924 (2003).

[70] G. Tuin, Ph.D. thesis, Eindhoven University of Technology (1995).

[71] A. Patist, V. Chhabra, R. Pagidipati, R. Shah, and D.O. Shah, Langmuir, 13, 432 (1997).

[72] M.A. Safarpour, A.A. Rafati, H. Gharibi and R. Sameti, J. Chin. Chem. Soc., 46, 983 (1999).

[73] H.V. Ly, Ph.D. thesis, University of California (2003). 
[74] S. Reekmans, H. Luo, M. Van der Auweraer, and F.C. De Schryver, Langmuir, 6, 628 (1990).

[75] S. Biggs, F. Grieser, Macromolecules, 28, 4877 (1995).

[76] Md.H. Kabir, M. Ishitobi, H. Kunieda, Colloid and Polymer Science, Springer-Verlag, Berlin (2002).

[77] H.V. Ly, and M.L. Longo, Biophysical Journal, 87, 1013 (2004).

[78] H. Surburg, J. Panten, Common Fragrance and Flavor Materials: Preparation, Properties, and Uses, Wiley-VCH, Wienheim (2006).

[79] D.J. Rowe, Chemistry and Technology of Flavors and Fragrances, CRC Press, Oxford, (2005).

[80] S.E. Frieberg, Q. Yin, P.A. Aikens, Int. J. of Cosm. Sci., 20, 355 (1998).

[81] E. Roduner, Muonium, An Ultra-Light Hydrogen Isotope, in "Isotope Effects in the Biological and Chemical Sciences" ed. A. Kohen and H.-H. Limbach, Dekker, New York, chapter 15 (2006).

[82] E. Roduner, P.W. Percival, D.G. Fleming, J. Hochmann, H. Fischer, Chem. Phys. Lett., 57, 37 (1978).

[83] A. Martyniak, H. Dilger, R. Scheuermann, I.M. Tucker, I. McKenzie, D. Vujošević and E. Roduner, Phys. Chem. Chem. Phys., 8, 4723 (2006).

[84] R. Scheuermann, I.M. Tucker, H. Dilger, E.J. Staples, G. Ford, S.B. Fraser, B. Beck, E. Roduner, Langmuir, 20, 2652 (2004).

[85] A. Martyniak, R. Scheuermann, H. Dilger, I.M. Tucker, T. Burkert, A.S.K. Hashmi, D. Vujošević and E. Roduner, Physica B, 374-375, 328 (2006).

[86] H. Dilger, A. Martyniak, R. Scheuermann, D. Vujošević, I.M. Tucker, I. McKenzie and E. Roduner, Physica B, 374-375, 317 (2006).

[87] http://en.wikipedia.org/wiki/Cosmic_ray.

[88] E. Roduner, Chem. Soc. Rev., 22, 337 (1993).

[89] S.F.J. Cox, J. Phys. C, 20, 3187 (1987).

[90] C.P. Slichter, Principles of Magnetic Resonance, Springer, Heidelberg (1990).

[91] P. Tregenna-Piggott, E. Roduner, E. Santos, Chem. Phys., 203, 317 (1996). 
[92] A.S.K. Hashmi, T. Burkert, J.W. Bats, and A. Martyniak, Z. Naturforsch., 61b, 179 (2006).

[93] C. Hashimoto, G. Cristobal, A. Nicolas, P. Panizza, J. Rouch and H. Ushiki, Meas. Sci. Technol., 12, 1 (2001).

[94] N. Lei, C.R. Safinya, D. Roux and K.S. Liang, Physical Review E, 56, 608 (1997).

[95] I. Koltover, S.H.J. Idziak, P. Davidson, Y. Li, C.R. Safinya, M. Ruths, S. Steinberg and J.N. Israelachvili, J. Phys. II France, 6, 893 (1996).

[96] MINUIT-Function Minimization and Error Analysis; CERN Program Library Entry D506 (CERN, Geneva, Switzerland).

[97] G. Bryant, K L. Koster and J. Wolfe, Seed Science Research, 11, 17 (2001).

[98] R. Scheuermann, I.M. Tucker, A.M. Creeth, H. Dilger, B. Beck, and E. Roduner, Phys. Chem. Chem. Phys., 4, 1510 (2002).

[99] T.M. Raschke, and M. Levitt, PNAS, 102, 6777 (2005).

[100] D. Marsh, PNAS, 98, 7777 (2001).

[101] D. Marsh, Eur. Biophys. J., 31, 559 (2002).

[102] D. Kurad, G. Jeschke, and D. Marsh, Biophysical Journal, 85, 1025 (2003).

[103] Sz. Vass, H. Grimm, I. Bányai, G. Meier, and T. Gilányi, J. Phys. Chem. B, 109, 11870 (2005).

[104] C. Ho and C. Stubbs, Biochemistry, 36, 10630 (1997).

[105] D. Vujošević, R. Scheuermann, H. Dilger, I.M. Tucker, A. Martyniak, I. McKenzie, E. Roduner, Physica B, 374-375, 295 (2006).

[106] D. Vujošević, H. Dilger, I. McKenzie, A. Martyniak, R. Scheuermann, E. Roduner, J. Phys. Chem. B, 111, 199 (2007).

[107] Y. Kondo, M. Abe, K. Ogino, H. Uchiyama, J.F. Scamehorn, E.E. Tucker, and S.D. Christian, Langmuir, 9, 899 (1993).

[108] E. Roduner, Hyperfine Interaction, 65, 857 (1990).

[109] R.E. Verrall, Chemical Society Reviews, 24, 136 (1995).

[110] B. Lee and G. Graziano, J. Am. Chem. Soc., 118, 5163 (1996).

[111] G. Graziano, Can. J. Chem., 78, 1233 (2000). 
[112] M. Heming, E. Roduner, I.D. Reid, P.W.F. Louwrier, J.W. Schneider, H. Keller, et al., Chem. Phys., 129, 335 (1989).

[113] S. Kreitzman, Chem. Phys., 152, 353 (1991).

[114] H. Dilger, E. Roduner, M. Stolmár, I.D. Reid, D.G. Fleming, D.J. Arseneau, J.J. Pan, M. Senba, M. Shelley, Hyperfine Interactions, 106, 137 (1997).

[115] http://chemdat.merck.de.

[116] G. Brigati, P. Franchini, M. Lucarini, G.F. Peduli, L. Valgimigli, Res. Chem. Intermed., 28, 131 (2002).

[117] Gaussian 98, Revision A. 7, M.J. Frisch, G.W. Trucks, H.B. Schlegel, G.E. Scuseria, M.A. Robb, J.R. Cheeseman, V.G. Zakrzewski, J.A. Montgomery, Jr., R.E. Stratmann, J.C. Burant, S. Dapprich, J.M. Millam, A.D. Daniels, K.N. Kudin, M.C. Strain, O. Farkas, J. Tomasi, V. Barone, M. Cossi, R. Cammi, B. Mennucci, C. Pomelli, C. Adamo, S. Clifford, J. Ochterski, G.A. Petersson, P.Y. Ayala, Q. Cui, K. Morokuma, D.K. Malick, A.D. Rabuck, K. Raghavachari, J.B. Foresman, J. Cioslowski, J.V. Ortiz, A.G. Baboul, B.B. Stefanov, G. Liu, A. Liashenko, P. Piskorz, I. Komaromi, R. Gomperts, R.L. Martin, D.J. Fox, T. Keith, M.A.Al. Laham, C.Y. Peng, A. Nanayakkara, C. Gonzalez, M. Challacombe, P.M.W. Gill, B. Johnson, W. Chen, M.W. Wong, J.L. Andres, M. Head-Gordon, E.S. Replogle, J.A. Pople, Gaussian, Inc., Pittsburgh PA (1998).

[118] J.A. Weil, J.R. Bolton, J.E. Wertz, Electron Paramagnetic Resonance, John Wiley \& Sons, New York, p. 125 (1994).

[119] A. Firouzi, D.J. Schaefer, S.H. Tolbert, G.D. Stucky and B.F. Chmelka, J. Am. Chem. Soc., 119, 9466 (1997).

[120] M.A. Firestone, D.M. Tiede, and S. Seifert, J. Phys. Chem. B, 104, 2433 (2000).

[121] E. Freyssingeas, D. Roux, F. Nallet, J. Phys.: Condens. Matter, 8, 2801 (1996).

[122] W. Helfrich, J. Phys: Condens. Matter, 6, A79 (1994).

[123] H. von Berlepsch, and R. de Vries, Eur. Phys. J. E, 1, 141 (2000).

[124] C.R. Safinya, D. Roux, G.S. Smith, S.K. Sinha, P. Dimon, N.A. Clark and A.M. Bellocq, Physical Review Letters, 57, 2718 (1986).

[125] P. Boltenhagen, O. Lavtentovich, M. Kleman, J. Phys. II France, 1, 1233 (1991). 
[126] R.I. Gearba, A.I. Bondar, B. Goderis, W. Bras, and D.A. Ivanov, Chem. Mater., 109, 4042 (2005).

[127] A.C. Riberio, B. Heinrich, C. Cruz, H.T. Nguyen, S. Diele, M.W. Schröder, and D. Guillon, Eur. Phys. J. E, 10, 143 (2003).

[128] G. Schmidt, S. Müller, P. Lindner, C. Schmidt, and W. Richtering, J. Phys. Chem. B, 102, 507 (1998).

[129] E. Eiser, F. Bouchama, M.B. Thathagar, and G. Rothenberg, Chem. Phys. Chem., 4 $526(2003)$.

[130] B.J. Balcom, N.O. Petersen, Biophysical Journal, 65, 630 (1993).

[131] F.M. Menger, M.E. Chlebowski, A.L. Galloway, H. Lu, V.A. Seredyuk, J.L. Sorrells, and H. Zhang, Langmuir, 21, 10336 (2005).

[132] F. Auguste, P. Barois, L. Fredon, B. Clin, E.J. Dufourc and A.M. Belloq, J. Phys. II France, 4, 2197 (1994).

[133] A. Martyniak, H. Dilger, I. McKenzie, R. Scheuermann, J. Lagerwall, E. Roduner, Colloids and Surfaces $A$, in press.

[134] Z. Gao, J.C.T. Kwak, R. Labonté, D.G. Maragoni and R.E. Wasylishen, Colloids and Surfaces, 45, 269 (1990). 